\title{
Um Método Numérico para a Solução de Problemas Inversos de Autovalores em Sistemas Discretos
}

\section{Juan Carlos Egaña Arancibia}

\author{
TESE APRESENTADA \\ $\mathrm{AO}$ \\ INSTITUTO DE MATEMÁTICA E ESTATÍSTICA \\ DA \\ UNIVERSIDADE DE SÃO PAULO \\ PARA OBTENÇÃO DO GRAU \\ DE \\ DOUTOR EM MATEMÁTICA APLICADA
}

\section{Área de Concentração: ANÁLISE NUMÉRICA Orientador: Prof. Dr. Luis Carlos de Castro Santos Co-orientador: Prof. Dr. Nelson Mugayar Kuhl}

Durante a elaboração deste trabalho, o autor recebeu apoio financeiro da CAPES e do Depto. de Matemática da Universidad Católica del Norte, Chile. 


\section{Um Método Numérico para a Solução de Problemas Inversos de Autovalores em Sistemas Discretos}

Este exemplar corresponde à redação final devidamente corrigida e defendida por Juan Carlos Egaña Arancibia e aprovada pela comissão julgadora.

São Paulo, 10 de Setembro de 1999.

Banca examinadora

- Prof. Dr. Luis Carlos de Castro Santos (IME-USP)

- Prof. Dr. Júlio Michael Stern

(IME-USP)

- Prof. Dr. Antonio Carlos Moretti (IMECC-UNICAMP)

- Prof. Dr. Norberto Mangiavacchi (ICMC-USP)

- Prof. Dr. Ricardo Soto Montero (UCN-CHILE) 
......depois de tantos anos de estudos quis responder a seguinte pergunta:

POR QUÉ ESTUDIAS?

e a Marcia, minha esposa, deu de presente para mim um cartão que dizia......

Un hombre puede estudiar por cinco razones:

Para saber. Para mostrar que sabe. Para obtener ganancias. Para instruir a otros. Para instruirse a si mismo.

Saber por saber, es curiosidad. Saber para mostrar que se sabe, es vanidad. Saber para conseguir dinero u honores, es comercio culpable. Saber para instruir a otros, es caridad. Saber para instruirse a sí mismo, es humildad.

Solamente los dos últimos no abusan de la ciencia, porque estudian para hacer el bien. 
A MI ESPOSA MARCIA. 


\section{Resumo}

Um problema inverso em vibração é estudado neste trabalho. O problema é chamado isolamento de frequências em sistemas discretos massa-mola (IFSMM), com aplicação em problemas de identificação, projeto estrutural e otimização. O problema IFSMM consiste em isolar as frequências naturais de um sistema massa-mola de uma certa banda de ressonância, gerando um novo espectro de frequências, e a partir deste, reconstruir um novo sistema minimizando o impacto de re-projeto relativo ao sistema inicial. Aqui é proposto um método numérico inverso de autovalor para resolver o problema IFSMM, onde um espectro intercalado $\mu$ é necessário. A fim de minimizar o impacto de re-projeto a escolha entre as soluções inversas é a que minimiza as mudanças nas propriedades físicas do sistema. O método é baseado numa estratégia de busca multidimensional original e eficiente, onde o $\mu$ é a incógnita. Esta estratégia implica um análise de sensibilidade, com relação ao espectro intercalado. A reconstrução inversa implica numa análise prévia da sensibildade de uma matriz de Jacobi $J(\mu)$. Neste trabalho, é proposto um método numérico direto que permite calcular a sensibilidade de $J(\mu)$ com exatidão numérica. Também um completo estudo de sensibildade em sistemas massa-mola é desenvolvido. São apresentados diferentes exemplos numéricos para mostrar a eficiência, robustez, e precisão do método numérico inverso de autovalor proposto, o qual permite identificar as mudanças que devem ser feitas no re-projeto de um sistema sujeito a ressonância.

\section{Abstract}

In this work an inverse problem in vibration is studied. The problem is named frequency isolation of spring-mass discrete systems (FISMS), with application in identification, structural design and optimization problems. The FISMS problem consists in isolating the natural frequencies of the spring-mass systems which lie in some resonance band, generating a new frequency spectra, and from it, reconstruct a new system minimizing the impact of re-design with respect to the initial system. A inverse eigenvalue numerical method is proposed to solve the FISMS problem, where an interlaced spectra $\mu$ is used. To minimize the impact of re-design the criteria of choice among the inverse solutions is the one which minimizes the changes in the physical properties of the system. The method is based in an original e efficient multidimensional search strategy. This strategy involves the sensitivity analysis in ralation to interlaced spectra. The inverse reconstruction include a sensitivity analysis of Jacobi matrix $J(\mu)$. In this work a direct numerical method is proposed which allows to compute the sensitivity $J(\mu)$ with numerical accuracy. Also a complete study of sensitivity of spring-mass systems is developed. Several numerical examples are presented to show the efficiency, robustness and accuracy of the inverse eigenvalue numerical method which allows the identifycations the changes that must be made in the re-design of a system in resonance. 


\section{Agradecimentos}

Na conclusão deste trabalho devo agradecer às pessoas que me apoiaram durante quatro anos no Brasil.

Primeiro agradeço a Deus por sentir que está presente em cada desafio empreendido na minha vida, embora acho que não mereço.

Meus amigos e colegas do instituto: Alice, Leandro, Carolina, Marcel, Gustavo, Nelson Chávez, Wálter Cárdenas, NelsonVN, o grande Santoyo, tio Choque, Xyoby e muitos outros.........

À comunidade chilena integrada por Carlitos, Robertão, Gonzalo, Francisco, Iván, Bernardo, Violeta, Mariano, Filidor, Ivo, meu grande amigo Cristian, e Luis (el loco) por motivar-me a começar o doutorado.

À minha grande amiga, e quase irmã, Claudia pela paciência e apoio recebido durante todos estos anos no Brasil. Também a meu caro colega e amigo Raúl, pelos "longos" anos de luta por conseguir este objetivo.

Àos professores do IME que influíram de alguma maneira na conclusão deste pós: Cyro, Roberto, e Saulo.

À Ricardo, meu ex-orientador do mestrado, pela confiança e apoio recebido desde o inicio deste doutorado.

À Luis Carlos e Nelson pela orientação e formação que recebi durante o desenvolvimento deste trabalho. Além disso, a paciência que significa trabalhar conmigo.........para falar verdade, são demais.

À minha familia......meus pais Lilian e Danilo, meu grande irmão e amigo "el Ruco", meu irmão Pancho, meus sobrinhos, meus sogros, meus cunhados, meus tios, etc.

.e especialmente à Marcia.....minha amiga, companheira, e esposa. Com ela compartilho este trabalho obrigado meu amor. 


\section{Sumário}

1 Introdução 1

2 Estrategia Unidimensional para o Isolamento de Frequências Naturais em Sistemas Discretos Massa-Mola $\quad 8$

2.1 Formulação do Problema . . . . . . . . . . . . . . . . . . . . . 8

2.2 Reconstrução de uma Matriz de Jacobi . . . . . . . . . . . . . . . . . . . 9

2.3 Reconstrução de Sistemas Massa-Mola . . . . . . . . . . . . . . . . . . . . . . 12

2.4 Estratégia Unidimensional para Solução Numérica do Problema IFSMM . . . 16

2.5 Resultados Computacionais . . . . . . . . . . . . . . . . . . 18

3 Análise de Sensibilidade de Matrizes de Jacobi 23

3.1 Aproximação por diferenças finitas . . . . . . . . . . . . . . . . . . 24

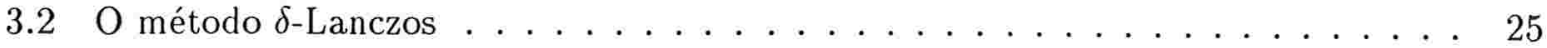

3.3 O método $\delta^{2}$-Lanczos . . . . . . . . . . . . . . . . . . 30

3.4 Resultados Computacionais . . . . . . . . . . . . . . . . 33

3.4.1 Gráficos das derivadas calculadas por $\delta$-Lanczos . . . . . . . . . . . . 34

3.4 .2 Comparação dos algoritmos com e sem ortogonalização . . . . . . . . . 34

3.4 .3 Consistência do algoritmo $\delta$-Lanczos $\ldots \ldots \ldots \ldots \ldots$

3.4.4 Derivada numérica exata obtida pelo algoritmo $\delta$-Lanczos . . . . . . . 38

3.4.5 Comparação dos métodos $\delta$-Lanczos e diferenças finitas $\ldots \ldots \ldots$. . . . 39

4 Análise de Sensibilidade de Sistemas Massa-Mola 41

4.1 Análise de sensibilidade das matrizes $M(\mu)$ e $K(\mu) \ldots \ldots \ldots \ldots \ldots$. . . . 42

4.2 Resultados Computacionais . . . . . . . . . . . . . . . . . . . 48

4.2.1 Gráficos das derivadas calculadas pelo algoritmo $4.1 \ldots \ldots$. . . . . . . 49

4.2 .2 Derivada numérica exata das massas e constantes de rigidez . . . . . . . 49 
4.2.3 Comparação do algoritmo 4.1 com diferenças finitas . . . . . . . . . . 54

5 Estratégia Multidimensional para o Isolamento de Frequências Naturais em Sistemas Discretos Massa-Mola 56

5.1 Estratégia multidimensional para matrizes de Jacobi . . . . . . . . . . . . . 58

5.2 Estratégia multidimensional para sistemas massa-mola . . . . . . . . . . 63

5.2 .1 Massa total constante . . . . . . . . . . . . . . . . . 63

5.2 .2 Massa total como variável independente . . . . . . . . . . . . 68

Conclusões

A Algoritmo tipo região de confiança para minimização com restrições de canalização Box

Referências Bibliográficas 


\section{Lista de Figuras}

1.1 Vibração transversal das partículas aderidas a uma corda fixa-fixa. . . . . . . . . 2

1.2 Vibração transversal das partículas aderidas a uma corda fixa-livre. . . . . . . . . . 2

1.3 Sistema massa-mola fixo-livre. . . . . . . . . . . . . . . . . . . . . . . 3

1.4 Sistema massa-mola modificado fixo-fixo. . . . . . . . . . . . . . . . . 4

1.5 Sistema massa-mola modificado com uma mola adicional fixo-fixo. . . . . . . . . 5

1.6 Sistema massa-mola modificado com parâmetros oscilatórios. . . . . . . . . . . 5

2.1 Banda de ressonância com centro c e raio $r \ldots \ldots \ldots \ldots$

2.2 Distribuição dos autovalores dentro da banda. . . . . . . . . . . . . . . . 17

2.3 Distribuição dos autovalores isolados da banda . . . . . . . . . . . . . . . 18

2.4 Comparação entre os sistemas original e inverso. Com frequências não isoladas; (a) sem restrição, (b) com restrição. . . . . . . . . . . . . . . . . . . . . 20

2.5 Comparação entre os sistemas original e inverso, impondo a restrição sob $m_{10} e$ $k_{1}$; (a) $\lambda_{6} \in B(4.5 ; 0.3)$, (a) $\lambda_{5}, \lambda \in B(3.5 ; 0.9) \ldots \ldots \ldots \ldots \ldots$

3.1 Gráficos das derivadas dos elementos diagonais e co-diagonais de uma matriz de Jacobi ordem $n=10$ com relação à primeira variável até a nona. . . . . . . . 35

3.2 Gráficos $\operatorname{dos} c_{j}=\log \left(e_{j+1}\right) / \log \left(e_{j}\right)$, onde $e_{j}$ denota o erro absoluto entre a solução exata e aproximada na j-ésima iteração do método de Newton para três valores iniciais diferentes. . . . . . . . . . . . . . . . . 38

4.1 Gráficos das derivadas das massas e constantes de rigidez do sistema com 10 graus de liberdade, ilustrado na Tabela 4.1, com relação às nove variáveis. . . . . . . . 51

4.2 Gráficos das derivadas das massas e constantes de rigidez do sistema com 10 graus de liberdade, ilustrado na Tabela 4.2, com relação às nove variáveis. . . . . . . . 52

4.3 Gráficos $d o s c_{j}=\log \left(e_{j+1}\right) / \log \left(e_{j}\right)$, onde $e_{j}$ denota o erro absoluto entre a solução exata e aproximada na j-ésima iteração do método de Newton para três valores inicias diferentes. . . . . . . . . . . . . . . . . . . . . . 
5.1 Comparação entre o sistema inicial e o final quando é isolado o segundo autovalor das bandas $\beta(26.0 ; 0.9), 3(26.0 ; 2.0)$, e $\beta(26.0 ; 4.0) \ldots \ldots \ldots \ldots$. . . . . . 76

5.2 Comparação entre o sistema inicial e o final quando é isolado o sexto autovalor das bandas $\beta(120.0 ; 3.4), \beta(120.0 ; 6.0)$, e $\beta(120.0 ; 12.0) . \ldots \ldots \ldots 77$

5.3 Comparação entre o sistema inicial e o final quando é isolado o nono autovalor das bandas $\beta(204.5 ; 1.1), \beta(204.5 ; 4.0)$, e $\beta(204.5 ; 12.0)$. . . . . . . . . 78

5.4 Comparação entre o sistema inicial e o final quando são isolados o segundo e terceiro autovalor das bandas $\beta(37.55 ; 12.4), \beta(37.55 ; 14.0)$, e $\beta(37.55 ; 15.0) . \ldots .80$

5.5 Comparação entre o sistema inicial e o final quando são isolados o quinto e sexto autovalor das bandas $\beta(111.0 ; 14.0), \beta(111.0 ; 20.0)$, e $\beta(111.0 ; 25.0) \ldots \ldots \ldots$. . . 81

5.6 Comparação entre o sistema inicial e o final quando são isolados o oitavo e nono autovalor das bandas $\beta(189.8 ; 15.8), \beta(189.8 ; 22.0)$, e $\beta(189.8 ; 26.0) \ldots \ldots$. . . . 82

5.7 Comparação entre o sistema inicial e o final quando são isolados o sétimo, oitavo e nono autovalor das bandas $\beta(148.7 ; 25.5), \beta(148.7 ; 29.0)$, e $\beta(148.7 ; 32.0) . \quad \ldots \quad 83$ 


\section{Capítulo 1}

\section{Introdução}

Os problemas inversos aparecem numa variedade importante de aplicações em física e engenharia. Esta lista inclui, entre outros, teoria de circuitos, projeto de controle, e teoria de vibração. Um problema é chamado problema inverso devido a sua relação com outro problema, chamado problema direto, e porque as incógnitas, no inverso, são os dados, conhecidos no direto, e viceversa.

O estudo da vibração livre infinitesimal de sistemas elásticos sem amortecimento é de grande interesse na teoria clássica de vibração. Um dos problemas diretos clássicos consiste em determinar as respostas de um sistema (frequências naturais, modos de vibração, etc) para alguma distribução de forças, onde os dados são as propriedades do sistema (distribuição de massas, coeficientes de elasticidade, etc). Enquanto que, no correspondente problema inverso, os dados são as respostas, e as incógnitas as propriedades do sistema. Assim os problemas inversos têm um valor prático para ser aplicados em mecânica e projeto estrutural e otimização [2], [10], [23], [24], [29], [38], [47], [48], [49], [50], [51], [19], [20], [21], [44], [8], [11]. Problemas de dimensão maior podem ser encontrados em [4], [39], [41], [42], [63], [64], [65].

Em geral, se alguma informação espectral (frequências naturais e/ou modos de vibração) é dada, pode existir ou não um sistema com as propriedades especificadas, se existe pode ser único ou vários sistemas. O principal interesse neste trabalho é uma classe de problemas inversos, a reconstrução de sistemas, os quais, num sentido geral, pertencem aos problemas de identificação de parâmetros. Os problemas inversos tratados aqui são exclusivamente aqueles que os dados espectrais proporcionam um e somente um sistema vibrante do tipo especificado com as propriedades espectrais dadas.

Gantmakher e Krein [18] consideraram o problema inverso de determinar as massas e posições de um conjunto de $n$ partículas aderidas a uma corda tensa, a partir das frequências de vibrações transversais da mesma. A corda é fixada nos extremos. Os dados consistem em dois espectros: 
o primeiro, $\left(\lambda_{i}\right)_{1}^{n}$, correspondente ao extremo direito fixo, como mostra a Fig. 1.1; e o segundo $\left(\mu_{i}\right)_{1}^{n}$, correspondente ao extremo direito deslizando-se livremente em uma varinha em ângulo reto à posição de equilibrio da corda, como na Fig. 1.2.

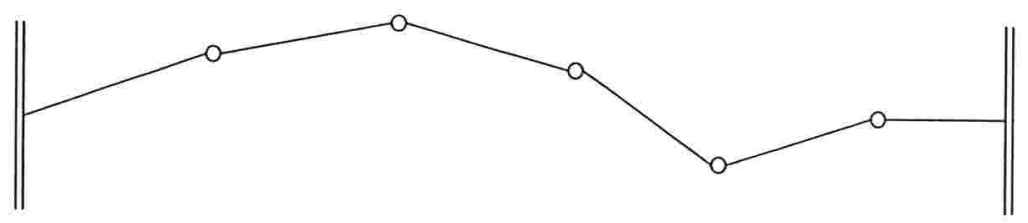

Figura 1.1: Vibração transversal das partículas aderidas a uma corda fixa-fixa.

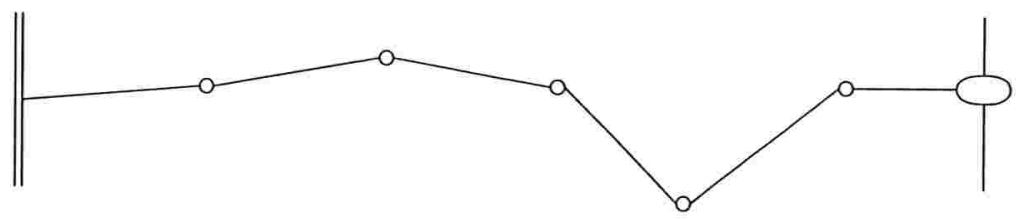

Figura 1.2: Vibração transversal das partículas aderidas a uma corda fixa-livre.

As equações que governam o problema da corda são matemáticamente semelhantes às que governam as vibrações de um sistema massa-mola com $n$ graus de liberdade com $n$ massas $m_{i}$ conectadas por molas lineares $\left(k_{i}\right)_{1}^{n}$, com extremo direito livre, como mostra a Fig. 1.3. Este sistema é caracterizado pela equação de autovalor generalizada

$$
(K-\lambda M) u=0,
$$

onde o autovetor $u$ está relacionado aos deslocamentos das massas. A matrizes $M$ e $K$ são diagonal e tridiagonal simétrica definida positiva, respectivamente. $M$ é chamada matriz de massa, e $K$ matriz de rigidez. A equação de autovalor generalizado 1.1 pode ser reduzida à forma clássica:

$$
(J-\lambda I) v=0,
$$

onde $v=M^{1 / 2} u$, e $J=M^{-1 / 2} K M^{-1 / 2}$ é uma matriz tridiagonal simétrica positiva definida com elementos co-diagonais negativos. Uma matriz tridiagonal simétrica será chamada matriz de Jacobi. Estas matrizes têm autovalores positivos distintos, o autovetor correspondente ao $i$-ésimo 
autovalor, em ordem ascendente, tem exatamente $i-1$ troca de sinais entre suas componentes (ver [26]).

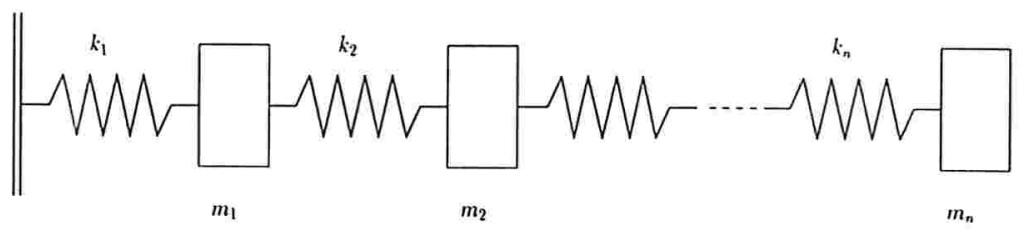

Figura 1.3: Sistema massa-mola fixo-livre.

A reconstrução de sistemas massa-mola pode ser realizada em duas partes; primeiro reconstruir a matriz $J$ de 1.2 , e a partir desta, reconstruir as matrizes $M$ e $K$. A teoria básica da existência e unicidade do problema inverso de reconstrução de uma matriz de Jacobi foi desenvolvida principalmente por Hoschstadt e Hald ([37], [35]). O primeiro demonstrou que existe no máximo uma matriz de Jacobi $J$ com a propriedade requerida, e tentou reconstruir esta única matriz, mas não consiguiu provar que seu método sempre leva a valores reais dos elementos codiagonais. Hald [35] apresentou outra construção e provou que, em teoria, este método sempre resultará em autovalores $\left(\lambda_{i}\right)_{1}^{n}$ de $J$, e $\left(\mu_{i}\right)_{1}^{n-1}$ correspondentes à submatriz principal superior $\tilde{J}$ de ordem $(n-1)$, obtida apagando a última linha e coluna de $J$, satisfazendo a propriedade de intercalamento:

$$
\lambda_{1}<\mu_{1}<\lambda_{2}<\cdots<\mu_{n-1}<\lambda_{n} .
$$

$\mathrm{Na}$ prática a construção tem uma falha devido a perda de precisão. Hald também provou que a construção de Hoschstadt levaria a valores reais dos elementos co-diagonais desde que a condição 1.3 seja satisfeita. Gray and Wilson [34] apresentaram uma construção indutiva da matriz $J$. Uma demonstração independente da unicidade do problema foi dada por Hald [35]. Posteriormente, foram desenvolvidos outros algoritmos numericamente estáveis e bem condicionados. Basicamente estes algoritmos são baseados em métodos que reduzem uma matriz real simétrica à forma tridiagonal tais como Householder, Given, e Lanczos (Ver [5], [6], [7], [14], [33]). Por exemplo, é possível calcular, a partir de $\left(\lambda_{i}\right)_{1}^{n}$ e $\left(\mu_{i}\right)_{1}^{n-1}$, as últimas componentes $v_{n}^{(i)}$ dos autovetores normalizados de $J$ na forma: 


$$
\left(v_{n}^{(i)}\right)^{2}=\frac{\prod_{j=1}^{n-1}\left(\mu_{j}-\lambda_{i}\right)}{\prod_{j=1, j \neq i}^{n-1}\left(\lambda_{j}-\lambda_{i}\right)}
$$

para $i=1,2, \ldots, n$ (Ver [6], [26], [32]). O conjunto de autovalores $\left(\mu_{i}\right)_{1}^{n-1}$ é conhecido como espectro intercalado, cujos valores são as raizes quadradas das frequências naturais de um sistema massa-mola modificado com a última massa fixa, como mostra a Fig. 1.4. O algoritmo de Lanczos pode ser aplicado adequadamente para reconstruir $J$ definindo a matriz diagonal $\Lambda=$ $\operatorname{diag}\left\{\lambda_{1}, \lambda_{2}, \ldots, \lambda_{n}\right\}$, e calculando um vetor unitário cujas componentes são dadas pela eq 1.4.

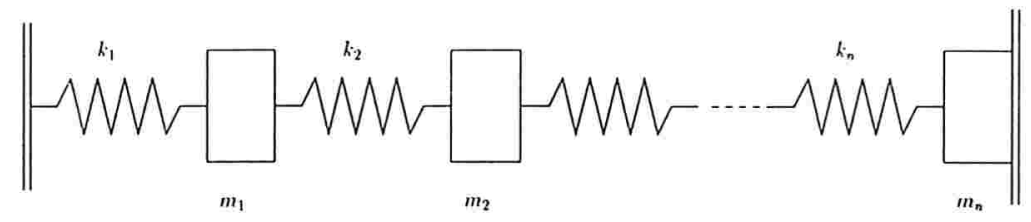

Figura 1.4: Sistema massa-mola modificado fixo-fixo.

A reconstrução de um sistema massa-mola também pode ser feita utilizando o espectro intercalado pertencente a um sistema modificado com outras condições de contorno. Por exemplo. fixar o extremo livre a um vínculo por meio de uma mola com rigidez $k_{n+1}$, como mostra a Fig. 1.5. Este sistema modificado está caracterizado por uma equação semelhante a 1.1, onde a matriz massa é a mesma, e a matriz rigidez difere no último elemento diagonal. Neste caso o espectro intercalado é $\left(\mu_{i}\right)_{1}^{n}$, onde a condição de intercalamento está dada por

$$
\lambda_{1}<\mu_{1}<\lambda_{2}<\cdots<\mu_{n-1}<\lambda_{n}<\mu_{n} .
$$

Outra condição de contorno foi imposta posteriormente por Ram [47]. Ele considerou o caso quando uma massa $m$ é aderida à massa $m_{n}$ e então é fixada a um vínculo por meio de uma mola de rigidez $k$ (Ver Fig. 1.6); a informação adicional da massa total é substituida pelos parâmetros osciladores $m$ e $k$, onde o sistema modificado terá autovalores $\left(\mu_{i}\right)_{1}^{n}$. As condições para obter um sistema fisicamente real são mais complexas que as 1.3 e 1.5, mas a condição de intercalamento dos espectros permanece (Ver [47]). A reconstrução de $M$ e $K$ é diferente às anteriores, embora a essência é a mesma, isto é, previamente à reconstrução das matrizes massa e rigidez uma matriz de Jacobi deve ser obtida a partir dos espectros $\left(\lambda_{i}\right)_{1}^{n},\left(\mu_{i}\right)_{1}^{n}$.

Todos os procedimentos descritos são relativos à reconstrução de uma matriz de Jacobi $J$. A reconstrução das matrizes massa e rigidez podem ser obtidas considerando a estrutura especial da matriz de rigidez. Os detalhes da reconstrução de $M$ e $K$ serão dados no Capítulo seguinte. 


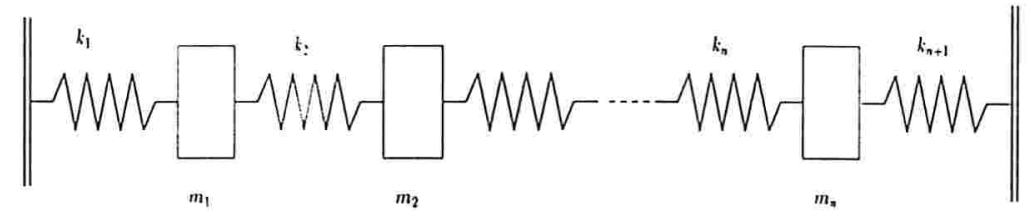

Figura 1.5: Sistema massa-mola modificado com uma mola adicional fixo-fixo.

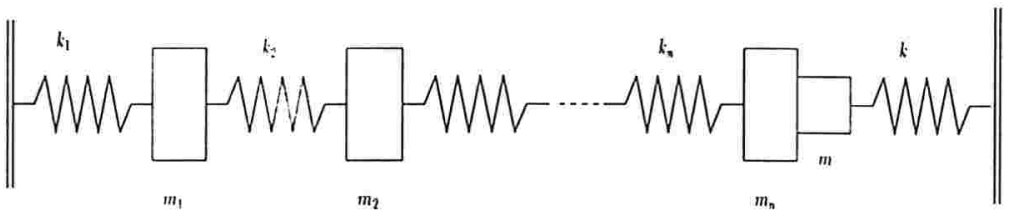

Figura 1.6: Sistema massa-mola modificado com parâmetros oscilatórios.

Uma aplicação dos problemas inversos em teoria estrutural estão relacionados aos problemas de detecção. Quando uma estrutura muda ou sofre algum dano, suas frequências também mudam. Este fato abre a possibilidade de encontrar a quantia e localização das mudanças numa estrutura a partir das mudanças nas frequências naturais.

Wu e Fricke [58], [60], [61] estimaram o tamanho e localização de um bloqueio num tubo usando frequências deslocamento. Wu e Fricke em [62] consideraram um problema semelhante para uma cavidade retangular. Shen e Taylor em [52] minimizaram um funcional definido como os quadrados das frequências e modos deslocamento para encontrar uma falha de uma viga. Wu em [59] reconstrói uma função falha integrada a partir das frequências deslocamento. Shepard e Milani [53], Hearn e Testa [36], Morassi [43], Davini, Gatti e Morassi [12], Davini, Morassi e Novore [13] consideraram a localização de dano em vigas e estruturas usando deslocamento de frequências.

Agora, um amplo e atual campo de pesquisa, pertencente aos problemas de detecção, são os problemas de identificação, e os problemas de projeto estrutural e otimização para vibração de estruturas. Toda máquina e estrutura, tais como pontes, edifícios, e aeronaves possuindo massa e rigidez experimentam vibração em algum grau, e seu projeto está diretamente relacionado com seu comportamento oscilatório. Quando um sistema oscila devido às forças inerentes ao sistema e sem qualquer força externa agindo sobre ele é dito que o sistema vibra livremente em uma ou mais de suas frequências naturais. A vibração forçada ocorre quando o sistema oscila pela ação de forças externas. Se tal excitação é oscilatória, o sistema é forçado a vibrar na frequência de excitação. Quando a frequência de excitação coincide ou fica perto de uma das frequências naturais do sistema, perigosamente podem ocorrer grandes oscilações encontrandose uma condição de ressonância. Esta condição é bem conhecida em problemas de vibração e 
devido à sua natureza catastrófica, especialmente quando esta ocorre nas frequências baixas, é analizada na primeira fase do projeto estrutrural.

Principalmente em construção civil, uma estrutura está sujeita a cargas periódicas as quais não foram préviamente planejadas. Por exemplo, máquinas rotativas ou dispositivos similares podem ser instalados posteriormente ao projeto original. Estes problemas usualmente são de baixa intensidade e diretamente relacionados à fadiga. O problema de isolamento de frequências consiste em remover as frequências naturais da estrutura de uma certa banda de ressonância , onde alguma fonte de vibração externa opera. Joseph em [38] apresenta um método para o projeto de uma estrutura cujas frequências baixas são especificadas. O método é baseado no método de Newton-Raphson e pode ser usado para isolar as frequências que ficam dentro de certas bandas, determinando algum sistema estrutural livre de ressonância. O método é limitado somente às frequências baixas do espectro. Para um número maior de frequências o método fica extremamente caro do ponto de vista computacional.

Um problema mais geral é isolar as frequências indesejadas de uma ou mais bandas, gerando um novo espectro de frequências, e a partir deste, reconstruir um novo sistema estrutural minimizando o impacto de re-projeto relativo ao sistema inicial. Neste trabalho é desenvolvido um método númerico inverso de autovalor para resolver o problema geral. A fim de minimizar o impacto de re-projeto a escolha entre as soluções inversas é aquela que minimiza as mudanças nas propriedades estruturais. Para tal um espectro auxiliar é necessário. Um modelo simples, um modelo massa-mola com $n$ graus de liberdade (Ver Fig. 1.3), é usado para demonstrar a viabilidade e potencial da técnica.

No Capítulo 2, descreve-se uma estratégia para remover as frequências de uma banda préviamente especificada, e também é proposto um algoritmo de busca unidimensional do espectro auxiliar para minimizar o impacto de re-projeto do sistema. Em sistemas massa-mola o espectro auxiliar é o espectro intercalado. A estratégia unidimensional é limitada somente ao isolamento de frequências centrais. Quando a banda contém frequências baixas, as soluções são altamente sensíveis. Além disso, a estratégia está restrita a valores pequenos de $n$, fazendo-se necessário o desenvolvimento de outras estratégias (Ver também [16]).

Uma outra tentativa para determinar uma solução do problema geral em sistemas massamola, é aplicar a chamada força bruta, isto é, minimizar uma função cujas incógnitas são as massas e constantes de rigidez de um sistema massa-mola. Esta técnica implica calcular, em cada passo do processo de minimização, os autovalores e autovetores do sistema, tornando-se um procedimento computacionalmente caro, embora seja seguro. Este fato, motiva o desenvolvimento de uma técnica mais sofisticada e eficiente do ponto de vista numérico.

Uma técnica novedosa e eficiente é uma generalização da estratégia unidimensional, isto é, definir uma função objetivo onde o espectro intercalado é a incógnita. Esta estratégia permite 
reconstruir, em cada passo do processo de minimização, um sistema $(M(\mu), K(\mu)$ com autovalores desejados até atingir o sistema objetivo. A nova metodologia reduz em $n+2$ o número de variáveis, com relacão à força bruta. O método de minimização usado é do tipo região de confiança o qual minimiza uma função de várias variáveis com restrição de canalização, como em 1.11. Na implementação, é necessário calcular, precisamente o vetor gradiente e a matriz Hessiana da função objetivo. Isto implica que, uma análise de sensibilidade das matrizes massa e rigidez com relação ao espectro intercalado é necessário. Como a reconstrução do sistema $(M(\mu), K(\mu))$ é precedida pela reconstrução de uma matriz de Jacobi $J(\mu)$, deve-se estudar a sensibilidade de $J(\mu)$. No Capítulo 3 é feito um estudo da sensibilidade de uma matriz de Jacobi com relação ao espectro intercalado. Aqui é desenvolvido um método numérico direto, baseado no algoritmo de Lanczos, que determina a sensibilidade de $J(\mu)$ com exatidão numérica. Este método é chamado método $\delta$-Lanczos (Ver também [15]). A mesma técnica é usada para estender o método $\delta$-Lanczos para derivadas de ordem mais alta. Posteriormente, no Capítulo 4 apresentamos um método numérico para determinar as sensibilidades das matrizes $M(\mu)$ e $K(\mu)$. Este método permite calcular as derivadas de primeira e segunda ordem determinando assim o vetor gradiente e a matriz Hessiana de $F(\mu)$, necessários para resolver o problema variacional $1.10,1.11$.

A unicidade da reconstrução inversa de um sistema massa-mola depende da especificação de um fator adicional, por exemplo, a massa total $m_{T}$ do sistema. Este fato, a princípio, sugere manter $m_{T}$ constante na busca do mínimo. Experimentos numéricos indicam que existem vários pontos mínimos locais da função objetivo entorno do mínimo, e provavelmente, os mínimos locais ficam presos em pontos estacionários, não permitindo encontrar uma direção de descida ao mínimo. Portanto, uma reformulação do problema, onde a massa total é considerada uma variável independente, é considerado depois, permitindo relaxar o processo de minimização na busca do mínimo. Os resultados numéricos obtidos da estratégia multidimensional são apresentados no Cap 5. Todos os exemplos considerados confirmam que a estratégia multidimensional é eficiente, a qual permite identificar as mudanças que devem ser feitas nas propriedades físicas de um sistema massa-mola sujeito a ressonância. Finalmente, no Cap 6 são estabelecidas algumas conclusões sugerindo linhas de pesquisa no futuro. 


\section{Capítulo 2}

\section{Estrategia Unidimensional para o Isolamento de Frequências Naturais em Sistemas Discretos Massa-Mola}

\subsection{Formulação do Problema}

O principal objetivo deste trabalho é desenvolver uma tecnologia eficiente e robusta para determinar uma solução numérica do seguinte problema:

PROBLEMA IFSMM: Isolamento de Frequências em Sistemas Massa-Mola

Considerar um sistema massa-mola vibrando longitudinalmente com $n$ massas $\bar{m}_{i}$ conectadas por molas lineares de rigidez $\left(\bar{k}_{i}\right)_{1}^{n}$. Este sistema será denotado pelo par $(\bar{M}, \bar{K})$, onde suas frequências naturais (ou simplesmente autovalores) $\left(\bar{\lambda}_{i}\right)_{1}^{n}$ são arranjados em ordem ascendente. Define-se a função diferenciável $H: \Re^{2 n} \longrightarrow \Re$ por

$$
H\left(m_{1}, m_{2}, \ldots, m_{n}, k_{1}, k_{2}, \ldots, k_{n}\right)=\sum_{j=1}^{n}\left(m_{j}-\bar{m}_{j}\right)^{2}+\sum_{j=1}^{n}\left(k_{j}-\bar{k}_{j}\right)^{2}
$$

onde as variáveis $\left(m_{j}, k_{j}\right)_{1}^{n}$ sao os parâmetros físicos de um sistema massa-mola $(M, K)$.

Dada uma banda de ressonância $\beta(c ; r) \subset \Re$ com centro $c$ e raio $r$ (Ver Fig 1.1) contendo um ou mais autovalores $\bar{\lambda}_{i}$, suponha-se que $\left(m_{j}^{*}, k_{j}^{*}\right)_{1}^{n}$ correspondem as massas e constantes de rigidez do sistema $\left(M^{*}, K^{*}\right)$, o problema de isolamento de frequências consiste em determinar um espectro $\left(\lambda_{i}^{*}\right)_{1}^{n}$, com $\lambda_{i}^{*}<\lambda_{i+1}^{*}$, correspondente a $\left(M^{*}, K^{*}\right)$ tal que as seguintes condições sejam satisfeita : 
(i) $\quad \lambda_{i}^{*} \notin \beta(c ; r), \forall i, i=1,2, \ldots, n, \mathrm{e}$

(2i) $\left(m_{1}^{*}, m_{2}^{*}, \ldots, m_{n}^{*}, k_{1}^{*}, k_{2}^{*}, \ldots, k_{n}^{*}\right)$ é um ponto mínimo de $H$.

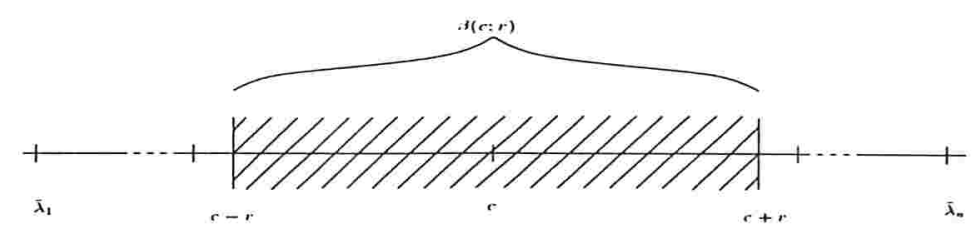

Figura 2.1: Banda de ressonância com centro $c$ e raio $r$

Este problema surgiu com a necessidade de identificar um sistema objetivo a partir dos autovalores, tendo uma motivação especial na aplicação em projeto estrutural. Isto é, tentar identificar quais são as mudanças nas propriedades estruturais que devem ser feitas numa estrutura sujeita a vibração. Trabalhos relacionados com este problema, mas aplicando tecnologias diferentes foram desenvolvidos por Udwadia, Garba, e Ghodsi [56], Torkamani e Ahmadi [55], e Joseph [38].

\subsection{Reconstrução de uma Matriz de Jacobi}

Neste Capítulo será descrito uma estratégia unidimensional para a solução do problema IFSMM. A técnica é baseada numa método inverso de autovalor para reconstruir um sistema massa-mola a partir dos autovalores. A reconstrução de um sistema massa-mola passa pela reconstrução prévia de uma matriz de Jacobi $J$. Isto será descrito com detalhes na Seção 2.3, mas um algoritmo de reconstrução de $J$ desenvolvido por Golub e Boley [31], e de Boor e Golub [14] é apresentado primeiro. O método está baseado no algoritmo de Lanczos que reduz qualquer matriz simétrica à forma tridiagonal, começando com a própria matriz e um certo vetor unitário. O método é brevemente discutido. Um tratamento completo do algoritmo de Lanczos é apresentado em [14], [26], [31], [32], [40], [45].

Supondo que $V=\left[v^{(1)}, v^{(2)}, \ldots, v^{(n)}\right]$ é a matriz de autovetores de $J$, onde as colunas de $V$ satisfazem a propriedade de ortogonalidade

$$
\left(v^{(i)}\right)^{T} v^{(j)}=\delta_{i j}
$$

A matriz $V$ satisfaz 


$$
J V=V \Lambda
$$

ou

$$
Q J=\Lambda Q
$$

onde $\Lambda=\operatorname{diag}\left\{\lambda_{1}, \lambda_{2}, \ldots, \lambda_{n}\right\}$, e $Q=\left[q^{(1)}, q^{(2)}, \ldots, q^{(n)}\right]=V^{T}$. Claramente, a eq 2.2 leva

$$
\left(q^{(i)}\right)^{T} q^{(j)}=\delta_{i j}
$$

onde o vetor $q^{(i)}$ é a $i$-ésima linha da matriz de autovetores de $J$. A matriz diagonal $\Lambda$ e a primeira (ou última) linha de $V$ formam os dados para o seguinte

ALGORITMO 2.1: (algoritmo de Lanczos) Dada a matriz diagonal $\Lambda=\operatorname{diag}\left\{\lambda_{1}, \lambda_{2}, \ldots, \lambda_{n}\right\}$ e $q^{(1)}$ (ou $q^{(n)}$ ) correspondente à primeira (ou última) linha da matriz de autovetores, este produz uma matriz $J$ e, ao mesmo tempo, constrói os autovetores ortogonais de $J$ :

Definir $b_{0}:=1, q^{(0)}:=0$

$$
\begin{aligned}
& a_{1}:=\left(q^{(1)}\right)^{T} \Lambda q^{(1)} \\
& \text { Para } j=1, \ldots, n-1 \\
& \quad r^{(j+1)}:=\left(\Lambda-a_{j} I\right) q^{(j)}-b_{j-1} q^{(j-1)} \\
& \quad b_{j}:=\left\|r^{(j+1)}\right\|_{2} \\
& q^{(j+1)}:=\frac{r^{(j+1)}}{b_{j}} \\
& \quad a_{j+1}:=\left(q^{(j+1)}\right)^{T} \Lambda q^{(j+1)}
\end{aligned}
$$

Quando a última linha da matriz de autovetores é especificada, o algoritmo 2.1 produz a matriz $J$ no sentido oposto, isto é, $a_{n}, b_{n-1}, a_{n-1}, b_{n-2}, \ldots, b_{2}, a_{2}, b_{1}, a_{1}$.

Com uma implementação adequada o algoritmo 2.1 tem um custo computacional de $O\left(n^{2}\right)$ operaçōes. Quando algum $b_{j}=0$ o algoritmo falha, mas devido à influência de erros de arredondamento, isso raramente ocorre na prática. Em teoria, o algoritmo gerará $q_{j}$ ortogonais, mas os vetores calculados podem ficar cada vez menos ortogonais quando $j$ é incrementado. Estes defeitos podem ser corrigidos re-ortogonalizando o novo vetor calculado $q^{(j+1)}$ em relação 
a todos os vetores prévios $q^{(j)}$, produzindo os vetores ortogonais com precisão de máquina. Este procedimento leva ao algoritmo chamado Lanczos com ortogonalização completa:

ALGORITMO 2.2:

Definir $b_{0}:=1, q^{(0)}:=0$

Determinar $P_{0}=I-2 z^{(0)}\left(z^{(0)}\right)^{T} /\left(z^{(0)}\right)^{T} z^{(0)} \quad$ tal que $\quad P_{0} r^{(1)}=e^{(1)}$

$a_{1}:=\left(q^{(1)}\right)^{T} \Lambda q^{(1)}$

Para $j=1, \ldots, n-1$

$$
\begin{aligned}
& r^{(j+1)}:=\left(\Lambda-a_{j} I\right) q^{(j)}-b_{j-1} q^{(j-1)} \\
& w:=\left(P_{j-1} \cdots P_{0}\right) r^{(j)}
\end{aligned}
$$

Determinar $P_{j}=I-2 z^{(j)}\left(z^{(j)}\right)^{T} /\left(z^{(j)}\right)^{T} z^{(j)}$ tal que

$$
\begin{aligned}
& \quad P_{j} w=\left(w_{1}, \ldots, w_{j}, b_{j}, 0, \ldots, 0\right)^{T} \\
& q^{(j+1)}:=\left(P_{0} \cdots P_{j}\right) e^{(j+1)} \\
& a_{j+1}:=\left(q^{(j+1)}\right)^{T} \Lambda q^{(j+1)}
\end{aligned}
$$

As matrizes $P_{j}$ são as matrizes de Householder do vetor $w$. O processo de ortogonalização completa eleva a complexidade a $O(n)$ operações. Portanto, o algoritmo 2.2 tem uma complexidade total de $O\left(n^{3}\right)$ operacões.

Certamente que os algoritmos 2.1 e 2.2 podem ser aplicados sempre que os vetores $q^{(1)}$ ou $q^{(n)}$ sejam conhecidos. Golub e Boley [31] mostraram que, uma vez conhecidos os autovalores $\left(\lambda_{i}\right)_{1}^{n}$ e $\left(\mu_{i}\right)_{1}^{n-1}$ satisfazendo a condição de intercalamento 1.3 , as componentes de $q^{(n)}$ podem ser construídas considerando os valores estacionários de

$$
\begin{gathered}
f=v^{T} J v, \\
\text { sujeito } a: v^{T} v=1 ; v^{T} c=0
\end{gathered}
$$

onde $c$ é algum vetor tal que $c^{T} c=1$. Eles provaram que se os valores estacionários de 2.5, 2.6 são prescritos como sendo os $\mu_{i}$, então as componentes do vetor $d=Q c$, satisfazem 


$$
d_{i}^{2}=\frac{\prod_{j=1}^{n-1}\left(\mu_{j}-\lambda_{i}\right)}{\prod_{j=1, j \neq i}^{n-1}\left(\lambda_{j}-\lambda_{i}\right)} .
$$

Se $c=e_{n} \equiv(0,0, \ldots, 1)^{T}$ então os valores estacionários de 2.5- 2.6 são precisamente os autovalores de $\tilde{J}$, e o vetor $d$ é a última linha de $V$ (ou última coluna de $Q$ ). Assim a fórmula para as últimas componentes dos autovetores está dada por

$$
\left(v_{n}^{(i)}\right)^{2} \equiv\left(q_{i}^{(n)}\right)^{2}=\frac{\prod_{j=1}^{n-1}\left(\mu_{j}-\lambda_{i}\right)}{\prod_{j=1, j \neq i}^{n-1}\left(\lambda_{j}-\lambda_{i}\right)}
$$

para $i=1,2, \ldots, n$.

\subsection{Reconstrução de Sistemas Massa-Mola}

Para descrever a estratégia unidimensional que determina uma solução numérica do problema IFSMM, formulado na Seção 2.1, será delineado um método de reconstrução de sistemas massamola a partir dos autovalores. Este método é considerado inverso desde que, na teoria clássica em vibração, o método direto consiste em determinar os autovalores e/ou autovetores do sistema dado.

Considerar um sistema massa-mola unidimensional consistindo de $n$ massas $m_{i}$ conectadas por molas lineares de rigidez $\left(k_{i}\right)_{1}^{n}$, onde a primeira mola é fixada e a última massa é livre (Ver Fig. 1.3). Este sistema é caracterizado pela equação de autovalor generalizada

$$
\left(K-\lambda_{i} M\right) u^{(i)}=0,
$$

onde os autovetores $u^{(i)}$ estão relacionados aos deslocamentos das massas. A matriz de rigidez tem a forma

$$
K=\left(\begin{array}{ccccc}
k_{1}+k_{2} & -k_{2} & & & \\
-k_{2} & k_{2}+k_{3} & -k_{3} & & \\
& \ddots & \ddots & \ddots & \\
& & -k_{n-1} & k_{n-1}+k_{n} & -k_{n} \\
& & & -k_{n} & k_{n}
\end{array}\right)
$$


e a matriz de massa $M=\operatorname{diag}\left\{m_{1}, m_{2}, \ldots, m_{n}\right\}$. Este sistema é fisicamente realizável se consiste de massas e constantes de rigidez positivas, isto é, $m_{i}>0$ e $k_{i}>0$ para $i=1,2, \ldots, n$. Estas propriedades implicam que $M$ e $K$ são matrizes definida positivas. Além disso, como $K$ é também tridiagonal simétrica, os autovalores são reais, positivos, e distintos (Ver [26]).

Antes de descrever o método inverso para a reconstrução do sistema $(M, K)$ a partir dos autovalores, a equação 2.9 será reduzida à forma clássica do problema de autovalor. Seja

$$
M=B B^{T}
$$

onde

$$
B=B^{T}=\operatorname{diag}\left\{m_{1}^{1 / 2}, m_{2}^{1 / 2}, \ldots, m_{n}^{1 / 2}\right\},
$$

introduz-se o vetor $v^{(i)}$ relacionado a $u^{(i)}$ por

$$
v^{(i)}=B^{T} u^{(i)}, \quad u^{(i)}=\left(B^{T}\right)^{-1} v^{(i)} .
$$

Se a equação 2.9 é pre-multiplicada por $B^{-1}$, obtém-se

$$
\left(J-\lambda_{i} I\right) v^{(i)}=0
$$

onde

$$
J=B^{-1} K\left(B^{T}\right)^{-1} .
$$

Assim, a matriz $J$ é tridiagonal simétrica definida positiva com os mesmos autovalores do par $(M, K)$. Claramente, a relação 2.15 implica que os elementos diagonais e co-diagonais de $J$ são positivos e estritamente negativos, respectivamente. Por outro lado, os polinôminos característicos das submatrizes principais de $J$ ficam invariantes sob transformações diagonalmente similares $D J D$, onde $D=\operatorname{diag}\left\{(-1)^{i}, i=1,2, \ldots, n\right\}$. Segue-se, portanto que, sem perda de geralidade, os elemento diagonais e co-diagonais serão assumidos estritamente positivos.

Gantmakher e Krein [18] provaram que as massas e constantes de rigidez do sistema $(M, K)$ podem ser reconstruídas unicamente se a seguinte informação é dada :

(1) os autovalores $\left(\lambda_{i}\right)_{1}^{n}$ de 2.9 ,

(2) os autovalores $\left(\mu_{i}\right)_{1}^{n-1}$ correspondentes ao sistema auxiliar com extremo direito fixo, como mostra a Fig. 1.4, 
(3) um fator adicional, por exemplo, a massa total do sistema $m_{T}=\sum_{i=1}^{n}\left(m_{i}\right)$.

A equação que caracteriza o sistema com extremo direito fixo é dada por

$$
\left(\widetilde{K}-\mu_{i} \widetilde{M}\right) \tilde{u}^{(i)}=0,
$$

onde as matrizes $\widetilde{M}$ e $\widetilde{K}$ são as submatrizes principais superiores que resultam ao eliminar a última linha e coluna das matrizes $M$ e $K$, respectivamente. Como na equação 2.9 , a equação anterior pode ser reduzida à equação de autovalor

$$
\left(\tilde{J}-\mu_{i} I\right) \tilde{v}^{i}=0
$$

onde $\tilde{J}$ é a submatriz principal superior de $J$.

A propriedade de intercalamento 1.3 , isto é,

$$
\lambda_{1}<\mu_{1}<\lambda_{2}<\cdots<\mu_{n-1}<\lambda_{n} .
$$

é condição necessária e suficiente para a existência de um sistema físicamente real, onde os autovalores $\left(\mu_{i}\right)^{n-1}$ são chamados espectro intercalado. A reconstrução do sistema é realizada em duas partes; primeiro a matriz $J$ de 2.14 é reconstruida usando o algoritmo de Lanczos 2.1 ou 2.2. Uma vez que $J$ é obtida, e considerando a relação 2.15 , a segunda parte consiste em separar as massas e constantes de rigidez de $J$.

Para descrever o procedimento de reconstrução das matrizes $M$ e $K$, considera-se uma forma particular da última, isto é,

$$
K(1,1, \ldots, 1)^{T}=\left(k_{1}, 0, \ldots, 0\right)^{T} .
$$

Reescrevendo 2.19 na forma

$$
B J B(1,1, \ldots, 1)^{T}=\left(k_{1}, 0, \ldots, 0\right)^{T},
$$

ou, equivalentemente,

$$
J\left(m_{1}^{1 / 2}, m_{2}^{1 / 2}, \ldots, m_{n}^{1 / 2}\right)^{T}=\left(k_{1} m_{1}^{-1 / 2}, 0, \ldots, 0\right)^{T},
$$

segue-se que, se $y=\left(y_{1}, y_{2}, \ldots, y_{n}\right)^{T} \neq 0$, é solução do sistema

$$
J y=e_{1},
$$


onde $e_{1}=(1,0, \ldots, 0)^{T}$, então

$$
\left(m_{1}^{1 / 2}, m_{2}^{1 / 2}, \ldots, m_{n}^{1 / 2}\right)^{T}=k_{1} m_{1}^{-1 / 2} y
$$

Isto implica que

$$
k_{1}^{2}=\frac{m_{i} m_{1}}{y_{i}^{2}}, i=1,2, \ldots, n .
$$

Assim,

$$
\frac{m_{1}}{m_{i}} y_{i}^{2}=\frac{m_{1}}{m_{n}} y_{n}^{2}, i=1,2, \ldots, n-1
$$

ou, equivalentemente,

$$
m_{i}=m_{n}\left(\frac{y_{i}}{y_{n}}\right)^{2}, i=1,2, \ldots, n-1
$$

Por outro lado, tem-se

$$
m_{T}=\sum_{i=1}^{n} m_{i} .
$$

Se $m_{T}$ é conhecida, então, pela relação 2.26 , a última massa do sistema é dada por

$$
m_{n}=\frac{m_{T}}{1+\sum_{i=1}^{n-1}\left(\frac{y_{i}}{y_{n}}\right)^{2}},
$$

e por tanto, a matriz de massa é totalmente determinada. Para determinar a matriz de rigidez pode usar-se a relação 2.15 , isto é,

$$
K=\left(B^{T}\right)^{-1} J B^{-1} .
$$

Resumindo, a reconstrução do sistema pode ser obtida através do seguinte

\section{ALGORITMO 2.3:}

Dados os espectros $\left(\lambda_{i}\right)_{1}^{n},\left(\mu_{i}\right)_{1}^{n-1}$, e a massa total $m_{T}$ do sistema:

1. Reconstruir a matriz de Jacobi $J$ a partir de $\left(\lambda_{i}\right)_{1}^{n}$ e $\left(\mu_{i}\right)_{1}^{n-1}$ (Ver os algoritmos 2.1 e 2.2 da Sação 2.2). 
2. Resolvendo o sistema 2.22 , determinar $\left(y_{i}\right)_{1}^{n}$.

3. Calcular $m_{n}$ de 2.28 .

4. Usando 2.26, encontrar $\left(m_{i}\right)_{1}^{n-1}$.

5. $M=\operatorname{diag}\left\{m_{1}, m_{2}, \ldots, m_{n}\right\}$ e $K=\left(B^{T}\right)^{-1} J B^{-1}$, onde $B$ é dada por 2.12 .

A reconstrução de um sistema massa-mola $(M, K)$ é baseado essencialmente na informação auxiliar do espectro intercalado $\mu=\left(\mu_{1}, \mu_{2}, \ldots, \mu_{n-1}\right)$. Isto implica que a reconstrução de $(M, K)$ pode considerar-se em função de $\mu$, isto é, fixado os autovalores $\left(\lambda_{i}\right)_{1}^{n}$ é possível obter diferentes sistemas $(M, K)$, para cada espectro intercalado $\mu$. A função $H$ de 2.1 considera as massas e constantes de rigidez como as incógnitas, então uma estratégia imediata para resolver o problema IFSMM é aplicar a chamada força bruta, isto é, determinar um sistema massa-mola e seus autovalores em cada passo de um processo de otimização até obter o sistema objetivo. Esta técnica é segura, mas tem uma eficiência computacional muito pobre, já que na avaliação da função objetivo e seu correspondente vetor gradiante é necessário o cálculo dos autovalores e autovetores de $(M, K)$. Estes fatos são discutidos em mais detalhe no Cap 5.

Uma estratégia diferente, de maioir eficiência computacional, é considerar uma função objetivo onde o espectro intercalado é incognita. Isto implica que, usando o método de reconstrução inversa em cada passo do processo de otimização usado, um sistema com os autovalores desejados será determinado até atingir o sistema objetivo. Esta nova técnica evidentemente diminue o número de variáveis da função objetivo em $n-1$, e não necessita o cálculo dos autovalores e autovetores em cada passo do processo de minimização. Na Seção seguinte descreve-se uma estrategia de busca unidimensional do espectro intercalado para avaliar o potencial da técnica. Os resultados numéricos confirmam a eficiência da nova técnica utilizada neste trabalho.

\subsection{Estratégia Unidimensional para Solução Numérica do Pro- blema IFSMM}

Dado um sistema massa-mola, nesta Seção, apresenta-se uma estratégia para isolar os autovalores que estão contidos numa banda préviamente estabelecida minimizando o impacto no re-projeto do sistema. A estrategia é baseada numa técnica inversa de autovalor e um conjunto de decisões empíricas dirigidas a minimizar o tempo computacional (Ver [16]). Primeiro, descreve-se um procedimento para isolar os autovalores indesejados, isto é, os que ficam dentro da banda, e posteriormente a estratégia que minimiza o impacto de re-projeto.

Sejam $(\bar{M}, \bar{K})$ um sistema massa-mola inicial com autovalores $\left(\bar{\lambda}_{i}\right)_{1}^{n}, \bar{\lambda}_{i}<\bar{\lambda}_{i+1}$, e $\beta(c ; r)$ uma banda de ressonância. Se alguns dos autovalores estão dentro de $\beta(c ; r)$, deve especificar-se novos 
valores para eles. Uma estratégia para determinar o novo espectro livre de ressonância, isto é, fora de $\beta(c ; r)$, consiste em re-definir apenas os autovalores que estão em $\beta(c ; r)$ mantendo o resto inalterado. Isto justifica-se porque grandes mudanças no espectro implicam grandes mudanças nos parâmetros físicos do sistema.

A fim de descrever o procedimento, primeiro é assumido que os autovalores que estão contidos na banda são distribuídos da seguinte forma:

$$
\bar{\lambda}_{i} \in\left\{\begin{array}{ll}
(c-r, c] & , i=k, k+1, \ldots, l \\
(c, c+r) & , i=l+1, l+2, \ldots, m
\end{array},\right.
$$

onde os índices $k$ e $m$ satisfazem $1<k \leq m<n$ (Ver Fig. 2.2).

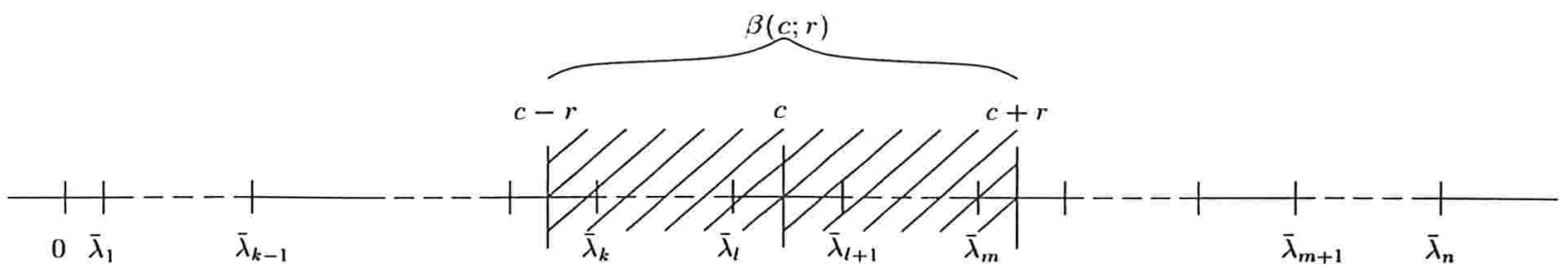

Figura 2.2: Distribuição dos autovalores dentro da banda.

Tendo em conta a ordem ascendente, uma possibilidade para isolar os autovalores indesejados é, remover os autovalores que estão dentro do intervalo $(c-r, c]$ pela relação

$$
\lambda_{i}^{*}=\bar{\lambda}_{k-1}+\frac{\left(\bar{\lambda}_{i}-c+r\right)\left(c-r-\bar{\lambda}_{k-1}\right)}{r},
$$

com $i=k, k+1, \ldots, l$. Enquanto que, para $i=l+1, l+2, \ldots, m$, a relação

$$
\lambda_{i}^{*}=c+r+\frac{\left(\bar{\lambda}_{m+1}-c-r\right)\left(\bar{\lambda}_{i}-c\right)}{r},
$$

é a que remove os autovalores que estão em $(c, c+r)$. Por outro lado, a fim de não provocar grandes mudanças no re-projeto do sistema, os autovalores que estão fora da banda são mantidos, isto é,

$$
\lambda_{i}^{*}=\bar{\lambda}_{i}, \forall i \neq j, j=k, k+1, \ldots, m .
$$


Assim, as eqs. 2.31, 2.32, 2.33 definem um procedimento para determinar o novo espectro $\left(\lambda_{i}^{*}\right)_{1}^{n}$ livre de ressonância, como mostra a Fig.2.3.

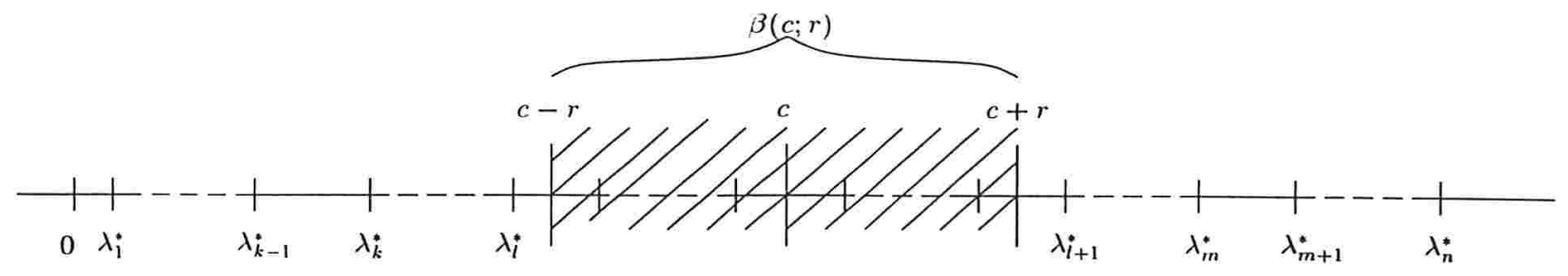

Figura 2.3: Distribuição dos autovalores isolados da banda.

Uma vez removido o espectro indesejável, é usada uma estratégia inversa para reconstruir um sistema $(M, K)$ com autovalores $\left(\lambda_{i}^{*}\right)_{1}^{n}$ minimizando o erro $L_{2}$ da reconstrução das massas e constantes de rigidez relativo ao sistema original $(\bar{M}, \bar{K})$. A fim de usar o algoritmo 2.1 , o espectro intercalado $\left(\mu_{i}\right)_{1}^{n}$ é calculado através de um algoritmo de busca unidimensional, isto é, para $i=1,2, \ldots, n-1$

$$
\mu_{i}=\lambda_{i}^{*}+s\left(\lambda_{i+1}^{*}-\lambda_{i}^{*}\right),
$$

com valores discretos $s=1 / t$, onde $t \in \Re$ é escolhido para satisfazer a condição (2i) do Problema IFSMM. Evidências numéricas mostram que, em certos casos, somente a última massa e primeira rigidez mudam significativamente, assim, como uma estratégia natural, a última massa e primeira rigidez são igualadas às originais uma vez que o processo de busca é terminado. No exemplo numérico discutido na Seção seguinte, mostra-se que os novos autovalores estão fora da banda indesejada.

\subsection{Resultados Computacionais}

Para mostrar a efetividade da estratégia descrita na Seção anterior um exemplo numérico simples é considerado. Seja $(\bar{M}, \bar{K})$ um sistema massa-mola com 10 graus de liberdade, com relação a vibrações longitudinais. Cada massa $\bar{m}_{i}$ será originalmente igual a 1.0, e cada mola com rigidez $\bar{k}_{i}$ igual a 2.0. Esta escolha proporciona um espectro inicial $\left(\bar{\lambda}_{i}\right)_{1}^{n}$, o qual é listado na primeira coluna das Tabelas 2.1, 2.2, 2.3, e 2.4 .

A eficiência do algoritmo é avaliada com três testes diferentes; primeiro, é considerada uma 
banda que não contém nenhum autovalor. Logo depois, uma contendo um autovalor, e finalmente uma com dois.

São definidos os erros

$$
E_{m}=\frac{\sum_{i=1}^{n}\left(m_{i}-\bar{m}_{i}\right)^{2}}{n}, E_{k}=\frac{\sum_{i=1}^{n}\left(k_{i}-\bar{k}_{i}\right)^{2}}{n},
$$

onde $\left(m_{i}, k_{i}\right)_{1}^{n}$ são os valores de massa e rigidez do sistema obtido depois que o procedimento é aplicado. Para o primeiro teste, o resultado ideal ocorre se não existe erro. A Fig. 2.4a mostra os erros $E_{m}$ e $E_{k}$ como funções de $t$, com os valores mínimos $E_{m^{*}}=2.09 \bar{\imath} \epsilon-02$ e $E_{k^{*}}=3.802 e-02$. $\mathrm{O}$ método inverso garante que o espectro do sistema recuperado é igual ao espectro objetivo, neste caso, o próprio $\bar{\lambda}_{i}$, pois nenhum autovalor foi isolado (Ver Tab. 2.1).

Experimentos numéricos mostram que quando são impostas as restrições $m_{10}=\bar{m}_{10}$ e $k_{1}=$ $\bar{k}_{1}$, encontram-se melhores valores mínimos $E_{m^{*}}=1.246 e-02$ e $E_{k^{*}}=1.4000 e-02$, ambos da ordem $1 \%$ (Ver Fig. 2.4b). Uma vez que, as restrições das massas e molas são aplicadas e o espectro do novo sistema é re-calculado, pode verificar-se que - comparando as últimas duas colunas da Tabela 2.2 com a primeira - o procedimento é aproximado.

Agora, suponha-se que é especificada uma banda com centro $c=4.5$ e raio $r=0.3$. Neste caso o autovalor $\bar{\lambda}_{6}$ deve ser removido. Usando as equações $2.31,2.32,2.33$, um novo espectro livre de ressonância é listado na segunda coluna da Tabela 2.3. Com este novo espectro objetivo e impondo as mesmas restrições anteriores sob a massa $m_{10}$ e a mola de rigidez $k_{1}$, obtém-se um valor mínimo para $E_{m^{*}}$ do ordem $10 \%$, e do $1 \%$ para $E_{k^{*}}$ (Fig. 2.5a).

Observa-se da Fig. $2.5 b$ que os erros $E_{m}$ e $E_{k}$ crescem quando o número de autovalores dentro da banda é melhor, neste caso $\bar{\lambda}_{5}$ e $\bar{\lambda}_{6}$ pertencem à banda $\beta(3.5 ; 0.9)$. Uma vez re-calculado o espectro pode verificar-se (Tabelas 2.3 e 2.4) que o procedimento assegura que o novo espectro está fora da banda.

Esta estratégia é limitada somente ao isolamento de frequências centrais. Quando $\beta(c ; r)$ contém autovalores extremos, as soluções são altamente sensíveis. Além disso, a estratégia está restrita para valores pequenos de $n$, fazendo-se necessário o desenvolvimento de outras estratégias. 


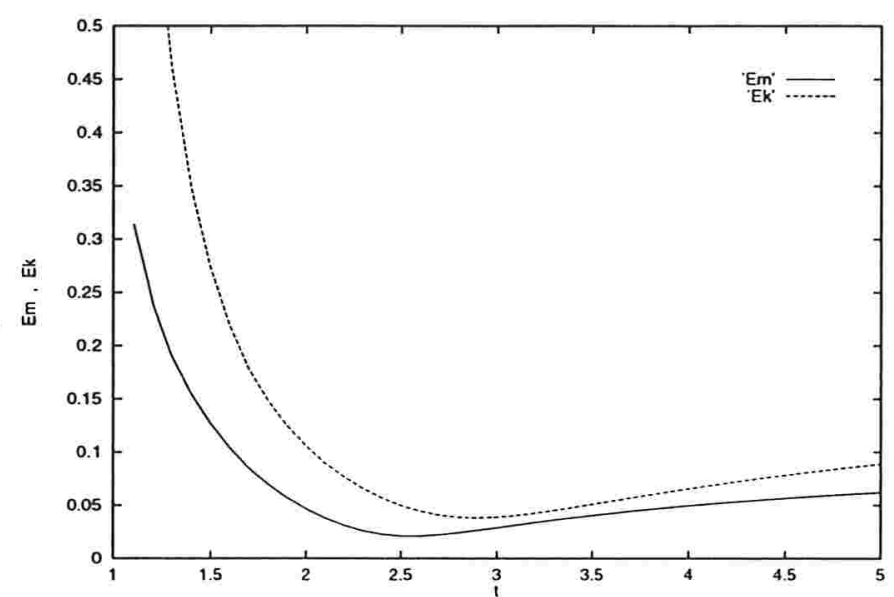

(a)

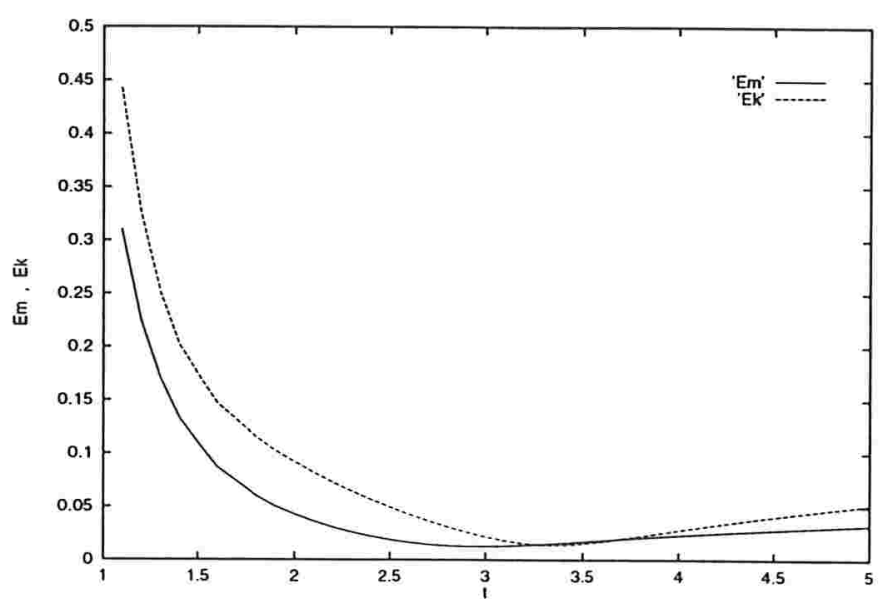

(b)

Figura 2.4: Comparação entre os sistemas original e inverso. Com frequências não isoladas; (a) sem restrição, (b) com restrição.

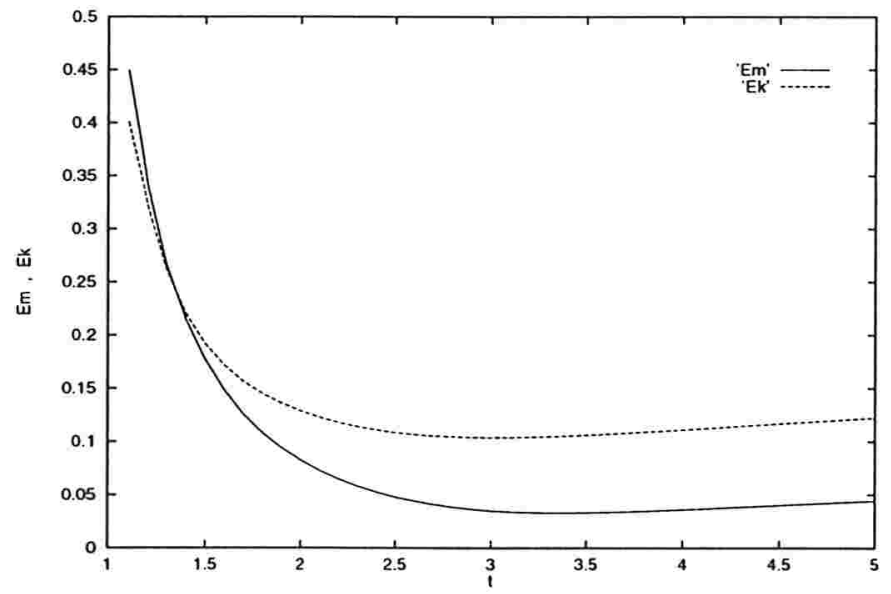

(a)

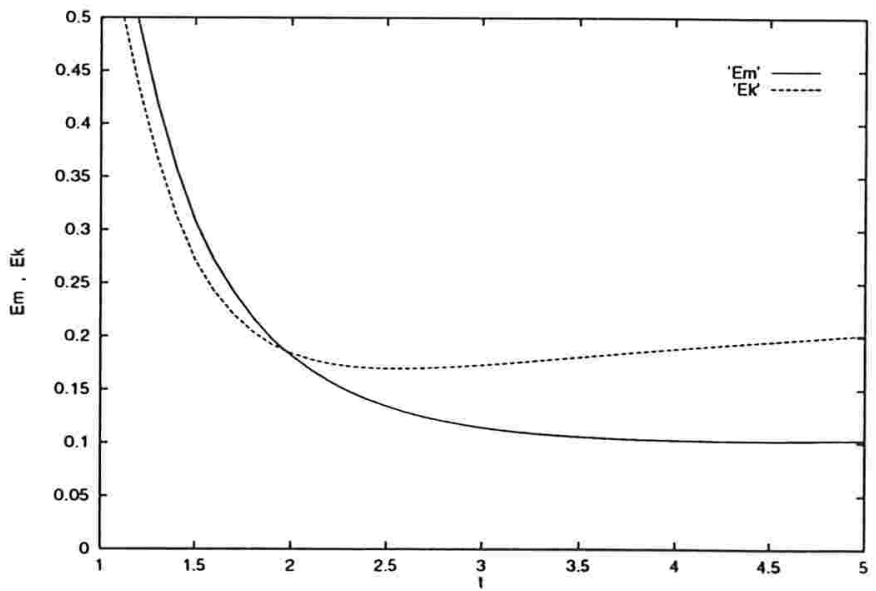

(b)

Figura 2.5: Comparação entre os sistemas original e inverso, impondo a restrição sob $m_{10}$ e $k_{1}$; (a) $\lambda_{6} \in B(4.5 ; 0.3)$, (a) $\lambda_{5}, \lambda \in B(3.5 ; 0.9)$. 
Tabela 2.1:

\begin{tabular}{cccc}
\hline \hline$i$ & $\bar{\lambda}_{i}$ & $\begin{array}{c}\lambda_{i}^{*}, t_{m^{*}}=2.5 \\
s r\end{array}$ & $\begin{array}{c}\lambda_{i}^{*}, t_{k^{*}}=2.9 \\
s r\end{array}$ \\
\hline \hline 1 & $4.467 \mathrm{e}-02$ & $4.467 \mathrm{e}-02$ & $4.467 \mathrm{e}-02$ \\
2 & 0.3961 & 0.3961 & 0.3961 \\
3 & 1.0677 & 1.0677 & 1.0677 \\
4 & 2.0000 & 2.0000 & 2.0000 \\
5 & 3.1099 & 3.1099 & 3.1099 \\
6 & 4.2989 & 4.2989 & 4.2989 \\
7 & 5.4613 & 5.4613 & 5.4613 \\
8 & 6.4939 & 6.4939 & 6.4939 \\
9 & 7.3049 & 7.3049 & 7.3049 \\
10 & 7.8222 & 7.8222 & 7.8222 \\
\hline \hline
\end{tabular}

Tabela 2.2:

\begin{tabular}{cccc}
\hline \hline$i$ & $\bar{\lambda}_{i}$ & $\begin{array}{c}\lambda_{i}^{*}, t_{m^{*}}=2.9 \\
c r\end{array}$ & $\begin{array}{c}\lambda_{i}^{*}, t_{k^{*}}=3.3 \\
c r\end{array}$ \\
\hline \hline 1 & $4.467 \mathrm{e}-02$ & $4.409 \mathrm{e}-02$ & $4.432 \mathrm{e}-02$ \\
2 & 0.3961 & 0.3929 & 0.3921 \\
3 & 1.0677 & 1.0538 & 1.0464 \\
4 & 2.0000 & 1.9703 & 1.9547 \\
5 & 3.1099 & 3.0652 & 3.0438 \\
6 & 4.2989 & 4.2434 & 4.2195 \\
7 & 5.4613 & 5.4005 & 5.3758 \\
8 & 6.4939 & 6.4327 & 6.4071 \\
9 & 7.3049 & 7.2455 & 7.2184 \\
10 & 7.8222 & 7.7409 & 7.7052 \\
\hline \hline
\end{tabular}


Tabela 2.3:

\begin{tabular}{ccccc}
\hline \hline$i$ & $\bar{\lambda}_{i}$ & $\lambda_{i}^{*}$ & $\begin{array}{c}\lambda_{i}^{*}, t_{m^{*}}=3.4 \\
c r\end{array}$ & $\begin{array}{c}\lambda_{i}^{*}, t_{k^{*}}=3.0 \\
c r\end{array}$ \\
\hline \hline 1 & $4.467 \mathrm{e}-02$ & $4.467 \mathrm{e}-02$ & $4.090 \mathrm{e}-02$ & $4.092 \mathrm{e}-02$ \\
2 & 0.3961 & 0.3961 & 0.3630 & 0.3643 \\
3 & 1.0677 & 1.0677 & 0.9792 & 0.9861 \\
4 & 2.0000 & 2.0000 & 1.8468 & 1.8566 \\
5 & 3.1099 & 3.1099 & 2.8879 & 2.8474 \\
6 & 4.2989 & 3.4693 & 3.4396 & 3.4538 \\
7 & 5.4613 & 5.4613 & 5.2574 & 5.3083 \\
8 & 6.4939 & 6.4939 & 6.3444 & 6.3795 \\
9 & 7.3049 & 7.3049 & 7.1887 & 7.2175 \\
10 & 7.8222 & 7.8222 & 7.6908 & 7.7229 \\
\hline \hline
\end{tabular}

Tabela 2.4:

\begin{tabular}{ccccc}
\hline \hline$i$ & $\bar{\lambda}_{i}$ & $\lambda_{i}^{*}$ & $\begin{array}{c}\lambda_{i}^{*}, t_{m^{*}}=4.4 \\
c r\end{array}$ & $\begin{array}{c}\lambda_{i}^{*}, t_{k^{*}}=2.6 \\
c r\end{array}$ \\
\hline \hline 1 & $4.467 \mathrm{e}-02$ & $4.467 \mathrm{e}-02$ & $3.821 \mathrm{e}-02$ & $3.920 \mathrm{e}-02$ \\
2 & 0.3961 & 0.3961 & 0.3357 & 0.3433 \\
3 & 1.0677 & 1.0677 & 0.8961 & 0.8965 \\
4 & 2.0000 & 2.0000 & 1.5606 & 1.4065 \\
5 & $\mathbf{3 . 1 0 9 9}$ & 2.3399 & 1.9417 & 2.1157 \\
6 & 4.2989 & 5.3421 & 4.5866 & 4.7828 \\
7 & 5.4613 & 5.4613 & 5.2012 & 5.2865 \\
8 & 6.4939 & 6.4939 & 6.2646 & 6.3927 \\
9 & 7.3049 & 7.3049 & 7.1283 & 7.2358 \\
10 & 7.8222 & 7.8222 & 7.6335 & 7.7505 \\
\hline \hline
\end{tabular}




\section{Capítulo 3}

\section{Análise de Sensibilidade de Matrizes de Jacobi}

Uma generalização do método apresentado no Capítulo anterior, o qual manipula outras frequências do espectro, consiste em formular um problema de otimização baseado num algoritmo de busca multidimensional para o espectro intercalado desconhecido. Para esta finalidade, define-se uma função objetivo

$$
F(\mu)=\sum_{j=1}^{n}\left(m_{j}(\mu)-\bar{m}_{j}\right)^{2}+\sum_{j=1}^{n}\left(k_{j}(\mu)-\bar{k}_{j}\right)^{2},
$$

onde $\mu=\left(\mu_{1}, \mu_{2}, \ldots, \mu_{n-1}\right)$ é o espectro intercalado relativo ao espectro objetivo $\left(\lambda_{i}^{*}\right)_{1}^{n},\left(m_{i}(\mu), k_{i}(\mu)\right)_{1}^{n}$ são as massas e constantes de rigidez reconstruídas a partir de $\left(\mu_{i}\right)_{1}^{n-1}$ e $\left(\lambda_{i}^{*}\right)_{1}^{n}$, e $\left(\bar{m}_{i}, \bar{k}_{i}\right)_{1}^{n}$ são as massas e constantes de rigidez originais. Uma vez definida a função objetivo, o problema é formulado como:

$$
\begin{aligned}
\text { Minimizar } & F(\mu) \\
\text { sujeito a : } & \lambda_{j}^{*}<\mu_{j}<\lambda_{j+1}^{*}, j=1,2, \ldots, n-1 .
\end{aligned}
$$

Como a reconstrução de um sistema $(M, K)$ é precedida pela reconstrução de uma matriz de Jacobi $J$, para resolver 3.2- 3.3, é necessário estudar a sensibilidade de $J$ com relação ao espectro intercalado $\mu$, o qual é o objetivo deste Capítulo. A solução de 3.2, 3.3 será tratada no Cap 5 . A análise de sensibilidade da matriz de Jacobi 


$$
J(\mu)=\left(\begin{array}{ccccc}
a_{1}(\mu) & b_{1}(\mu) & & & \\
b_{1}(\mu) & a_{2}(\mu) & b_{3}(\mu) & & \\
& \ddots & \ddots & \ddots & \\
& & b_{n-2}(\mu) & a_{n-1}(\mu) & b_{n-1}(\mu) \\
& & & b_{n-1}(\mu) & a_{n}(\mu)
\end{array}\right),
$$

analiticamente dependente nas $(n-1)$-variáveis $\mu_{1}, \mu_{2}, \ldots, \mu_{n-1}$, é o estudo de como os elementos da matriz são afetados pelas mudanças nas variáveis. Tal informação é muito importante para resolver o problema variacional $3.2,3.3$, e em geral para projeto estrutural e otimização.

Nosso objetivo neste Capítulo é calcular a derivada exata da matriz $J(\mu)$, em relação a cada variável $\mu_{k}, k=1,2, \ldots, n-1$. Neste trabalho uma derivada é considerada exata exceto por erros de arredondamento. A derivada parcial da matriz $J(\mu)$ com relação a uma variável $\mu_{k}$ é denotada por $\delta_{k} J(\mu)$, onde os elementos diagonais e co-diagonais de $\delta_{k} J(\mu)$ são $\delta_{k} a_{j}(\mu) \equiv \frac{\partial a_{j}(\mu)}{\partial \mu_{k}}$ e $\delta_{k} b_{j}(\mu) \equiv \frac{\partial b_{j}(\mu)}{\partial \mu_{k}}$, respectivamente.

Nesta Seção é discutido como obter as derivadas exatas e aproximadas de $J(\mu)$. A aproximação por diferenças finitas e suas implicações são descritas primeiro. Logo depois, uma extensão do método de Lanczos para calcular a matriz $\delta_{k} J(\mu)$, chamado método $\delta$-Lanczos é discutido (Ver também [15]).

\subsection{Aproximação por diferenças finitas}

A aproximação por diferenças centradas de segunda ordem é usada para aproximar $\delta_{k} J(\mu)$. Se cada $a_{j}(\mu), b_{j}(\mu)$, calculado pelo algoritmo de Lanczos com ou sem ortogonalização, são denotados por uma função $f_{j}(\mu)$ das variáveis $\mu_{k}$, a aproximação por diferenças centradas $\frac{\Delta f_{j}(\mu)}{\Delta \mu_{k}}$ para a sensibilidade $\delta_{k} f_{j}(\mu) \equiv \frac{\partial f_{j}(\mu)}{\partial \mu_{k}}$ é dada por

$$
\delta_{k} f_{j}(\mu) \simeq \frac{f_{j}\left(\mu_{1}, \ldots, \mu_{k}+\Delta \mu_{k}, \ldots, \mu_{n-1}\right)-f_{j}\left(\mu_{1}, \ldots, \mu_{k}-\Delta \mu_{k}, \ldots, \mu_{n-1}\right)}{2 \Delta \mu_{k}} .
$$

A matriz $\delta_{k} J(\mu)$ aproximada pelo esquema por diferenças finitas centradas usando o algoritmo 2.1 (sem ortogonalização) requer aproximadamente $O\left(n^{2}\right)$ operaçòes, enquanto o custo computacional é incrementado a $O\left(n^{3}\right)$ operações se a ortogonalização é usada. Por outro lado, se a derivada da matriz $J(\mu)$ é procurada para as $(n-1)$ variáveis, este método requer $(n-1)$ iterações adicionais do algoritmo 2.1 ou 2.2. Portanto, o trabalho computacional total é da ordem $O\left(n^{4}\right)$ operações. O trabalho computacional seria menor se a matriz $\delta_{k} J(\mu)$ fosse approximada por um esquema diferenças finitas de primeira ordem. 
Uma dificuldade da aproximação por diferenças finitas é a escolha de $\Delta \mu_{k}$. Devido à restrição de canalização 3.3 , em cada passo iterativo de um método de minimização para resolver 3.2, 3.3 deve ser determinado o valor ótimo $\Delta \mu_{k}$. Resultados numéricos da aproximação por diferenças finitas são apresentados na Seção 3.2, e estes resultados são usados como referências para comparação com o método da Seção seguinte.

\section{$3.2 \mathrm{O}$ método $\delta$-Lanczos}

A extensão do método de Lanczos para calcular as derivadas da matriz de Jacobi $J(\mu)$ em relação a $\mu_{k}, k=1,2, \ldots, n-1$ será chamado método $\delta$-Lanczos. Este método recupera as matrizes $\delta_{k} J(\mu)$ quando são especificados a matriz diagonal $\Lambda=\operatorname{diag}\left\{\lambda_{1}, \lambda_{2}, \ldots, \lambda_{n}\right\}$ e $q^{(1)}(\mu)$ (ou $q^{(n)}(\mu)$ ) a primeira (ou última) linha da matriz de autovetores.

Supondo que a primeira coluna da eq 2.3 é conhecida, onde $Q$ depende de $\mu$, tem-se

$$
a_{1}(\mu) q^{(1)}(\mu)+b_{1}(\mu) q^{(2)}(\mu)=\Lambda q^{(1)}(\mu)
$$

de maneira que

$$
a_{1}(\mu)=\left(q^{(1)}(\mu)\right)^{T} \Lambda q^{(1)}(\mu)
$$

já que $\left(q^{(1)}(\mu)\right)^{T} q^{(2)}(\mu)=0$.

Diferenciando a eq 3.7 com relação a $\mu_{k}$ resulta em

$$
\delta_{k} a_{1}(\mu)=\left(\delta_{k} q^{(1)}(\mu)\right)^{T} \Lambda q^{(1)}(\mu)+\left(q^{(1)}(\mu)\right)^{T} \Lambda \delta_{k} q^{(1)}(\mu)
$$

onde $\delta_{k} q^{(1)}(\mu)$ é obtida diferenciando-se o vetor $q^{(1)}(\mu)$, o qual é conhecido. Agora, como $\Lambda$ é uma matriz diagonal, a equação anterior pode ser escrita como

$$
\delta_{k} a_{1}(\mu)=2\left(\delta_{k} q^{(1)}(\mu)\right)^{T} \Lambda q^{(1)}(\mu)
$$

Por outro lado, definindo

$$
\begin{aligned}
r^{(2)}(\mu) & =\Lambda q^{(1)}(\mu)-a_{1}(\mu) q^{(1)}(\mu) \\
& =\left(\Lambda-a_{1}(\mu) I\right) q^{(1)}(\mu),
\end{aligned}
$$

e fazendo uso da eq $3.6, b_{1}(\mu)$ deve ser escolhido de maneira 


$$
b_{1}(\mu)=\left\|r^{(2)}(\mu)\right\|_{2},
$$

e então

$$
q^{(2)}(\mu)=\frac{r^{(2)}(\mu)}{b_{1}(\mu)}
$$

onde $\|\cdot\|_{2}$ denota a norma Euclidiana. Portanto, diferenciando as equações $3.9,3.10$, e 3.11, obtém-se

$$
\begin{aligned}
& \delta_{k} r^{(2)}(\mu)=\left(\Lambda-a_{1}(\mu) I\right) \delta_{k} q^{(1)}(\mu)-\delta_{k} a_{1}(\mu) q^{(1)}(\mu), \\
\delta_{k} b_{1}(\mu)= & \frac{r_{1}^{(2)}(\mu) \delta_{k} r_{1}^{(2)}(\mu)+r_{2}^{(2)}(\mu) \delta_{k} r_{2}^{(2)}(\mu)+\cdots+r_{n}^{(2)}(\mu) \delta_{k} r_{n}^{(2)}(\mu)}{\left(\left(r_{1}^{(2)}(\mu)\right)^{2}+\left(r_{2}^{(2)}(\mu)\right)^{2}+\cdots+\left(r_{n}^{(2)}(\mu)\right)^{2}\right)^{1 / 2}} \\
= & \frac{\left(r^{(2)}(\mu)\right)^{T} \delta_{k} r^{(2)}(\mu)}{\left\|r^{(2)}(\mu)\right\|_{2}} \\
= & \frac{\left(r^{(2)}(\mu)\right)^{T} \delta_{k} r^{(2)}(\mu)}{b_{1}(\mu)},
\end{aligned}
$$

e

$$
\delta_{k} q^{(2)}(\mu)=\frac{b_{1}(\mu) \delta_{k} r^{(2)}(\mu)-\delta_{k} b_{1}(\mu) r^{(2)}(\mu)}{\left(b_{1}(\mu)\right)^{2}}
$$

Uma vez que são achados $\delta_{k} a_{1}(\mu), \delta_{k} b_{1}(\mu)$, o resto dos elementos da matriz $\delta_{k} J(\mu)$ são determinados. Isto é, a $j$-ésima coluna da eq 2.3 é:

$$
b_{j-1}(\mu) q^{(j-1)}(\mu)+a_{j}(\mu) q^{(j)}(\mu)+b_{j}(\mu) q^{(j+1)}(\mu)=\Lambda q^{(j)}(\mu)
$$

onde $q^{(j)}(\mu)$ foi determinado no passo prévio de maneira que $\left(q^{(j)}(\mu)\right)^{T} q^{(j-1)}(\mu)=0$. Isto implica que

$$
a_{j}(\mu)=\left(q^{(j)}(\mu)\right)^{T} \Lambda q^{(j)}(\mu)
$$

e portanto

$$
\delta_{k} a_{j}(\mu)=2\left(\delta_{k} q^{(j)}(\mu)\right)^{T} \Lambda q^{(j)}(\mu)
$$

Além disso, definindo 


$$
\begin{aligned}
r^{(j+1)}(\mu) & =\Lambda q^{(j)}(\mu)-b_{j-1}(\mu) q^{(j-1)}(\mu)-a_{j}(\mu) q^{(j)}(\mu) \\
& =\left(\Lambda-a_{1}(\mu) I\right) q^{(j)}(\mu)-b_{j-1}(\mu) q^{(j-1)}(\mu),
\end{aligned}
$$

o elemento $b_{j}(\mu)$ é igual a

$$
b_{j}(\mu)=\left\|r^{(j+1)}(\mu)\right\|_{2},
$$

e

$$
q^{(j+1)}(\mu)=\frac{r^{(j+1)}(\mu)}{b_{j}(\mu)}
$$

Finalmente, diferenciando as três últimas equações, obtém-se

$$
\begin{gathered}
\delta_{k} r^{(j+1)}(\mu)=\left(\Lambda-a_{j}(\mu) I\right) \delta_{k} q^{(j)}(\mu)-\delta_{k} a_{j}(\mu) q^{(j)}(\mu) \\
-\delta_{k} b_{j-1}(\mu) q^{(j-1)}(\mu)-b_{j-1}(\mu) \delta_{k} q^{(j-1)}(\mu), \\
\delta_{k} b_{j}(\mu)=\frac{\left(r^{(j+1)}(\mu)\right)^{T} \delta_{k} r^{(j+1)}(\mu)}{b_{j}(\mu)},
\end{gathered}
$$

$\mathrm{e}$

$$
\delta_{k} q^{(j+1)}(\mu)=\frac{b_{j}(\mu) \delta_{k} r^{(j+1)}(\mu)-\delta_{k} b_{j}(\mu) r^{(j+1)}(\mu)}{\left(b_{j}(\mu)\right)^{2}} .
$$

Este procedimento, levado a cabo para $j=2,3, \ldots, n-1$ é chamado método $\delta$-Lanczos e pode resumir-se no seguinte

ALGORITMO 3.1: Dada uma matriz diagonal $\Lambda=\operatorname{diag}\left\{\lambda_{1}, \lambda_{2}, \ldots, \lambda_{n}\right\}$ de ordem $n$ e a primeira (ou última) linha da matriz de autovetores, este produz as matrizes $\delta_{k} J(\mu)$ e $\delta_{k} Q(\mu)$ tal que $\delta_{k} J(\mu)=\left(\delta_{k} Q(\mu)\right)^{T} \Lambda Q(\mu)+(Q(\mu))^{T} \Lambda \delta_{k} Q(\mu)$ :

Calcular $q^{1}(\mu), a_{1}(\mu)$, e $b_{j}(\mu), r^{j+1}(\mu), q^{j+1}(\mu), a_{j+1}(\mu), j=1,2, \ldots, n-1$ usando o algoritmo de Lanczos 2.1

$$
\text { Para } k=1, \ldots, n-1
$$

Calcular $\delta_{k} q^{(1)}(\mu)$ 


$$
\begin{aligned}
& \begin{array}{l}
\delta_{k} a_{1}(\mu):=2\left(\delta_{k} q^{(1)}(\mu)\right)^{T} \Lambda q^{(1)}(\mu) \\
\text { Para } j=1, \ldots, n-1 \\
\delta_{k} r^{(j+1)}(\mu):=\left(\Lambda-a_{j}(\mu) I\right) \delta_{k} q^{(j)}(\mu)-\delta_{k} a_{j}(\mu) q^{(j)}(\mu)-\delta_{k} b_{j-1}(\mu) q^{(j-1)}(\mu) \\
\quad-b_{j-1}(\mu) \delta_{k} q^{(j-1)}(\mu) \\
\delta_{k} b_{j}(\mu):=\frac{\left(r^{(j+1)}(\mu)\right)^{T} \delta_{k} r^{(j+1)}(\mu)}{b_{j}(\mu)} \\
\delta_{k} q^{(j+1)}(\mu):=\frac{b_{j}(\mu) \delta_{k} r^{(j+1)}(\mu)-\delta_{k} b_{j}(\mu) r^{(j+1)}(\mu)}{\left(b_{j}(\mu)\right)^{2}} \\
\delta_{k} a_{j+1}(\mu):=2\left(\delta_{k} q^{(j+1)}(\mu)\right)^{T} \Lambda q^{(j+1)}(\mu)
\end{array}
\end{aligned}
$$

Este algoritmo primeiro constrói os elementos da matriz $J(\mu)$, com um custo computacional de $O\left(n^{2}\right)$ operações. Uma vez construída a matriz $J(\mu)$, o algoritmo recupera cada matriz $\delta_{k} J(\mu)$ com aproximadamente $O\left(n^{2}\right)$ operações. Isto implica que são requeridas aproximadamente $O\left(n^{2}(n-1)\right)$ operações para calcular as matrizes $\delta_{k} J(\mu)$, para cada $k=1,2, \ldots, n-1$. Assim o trabalho total é $O\left(n^{3}\right)$. Se a última linha da matriz de autovetores é calculada, o procedimento recupera os elementos de $\delta_{k} J(\mu)$ no sentido oposto.

Como a reconstrução de $J(\mu)$ é baseada no algoritmo 2.1 , a perda de ortogonalidade dos vetores $q^{(j)}(\mu)$ podem levar a uma análise de sensibilidade incorreta. A dificuldade na diferenciação do algoritmo de Lanczos com ortogonalização é calcular $\delta_{k} b_{j}(\mu)$ e $\delta_{k} q^{(j)}(\mu)$, porque isto eleva o trabalho computacional, já que $\delta_{k} b_{j}(\mu)$ é a $(j+1)$-ésima componente do vetor $\delta_{k} P_{j}(\mu) w(\mu)+P_{j}(\mu) \delta_{k} w(\mu)$, onde

$$
\begin{aligned}
\delta_{k} w(\mu)= & \left(\delta_{k} P_{j-1}(\mu) P_{j-2}(\mu) \cdots P_{0}(\mu) r_{j}(\mu)\right) \\
& +\left(P_{j-1}(\mu) \delta_{k} P_{j-2}(\mu) \cdots P_{0}(\mu) r_{j}(\mu)\right) \\
& +\cdots+\left(P_{j-1}(\mu) P_{j-2}(\mu) \cdots P_{0}(\mu) \delta_{k} r_{j}(\mu)\right) .
\end{aligned}
$$

Da mesma forma,

$$
\begin{aligned}
\delta_{k} q^{(j)}(\mu)= & \left(\delta_{k} P_{0}(\mu) P_{1}(\mu) \cdots P_{j}(\mu) e_{j+1}\right)+\left(P_{0}(\mu) \delta_{k} P_{1}(\mu) \cdots P_{j}(\mu) e_{j+1}\right) \\
& +\left(P_{0}(\mu) P_{1}(\mu) \cdots \delta_{k} P_{j}(\mu) e_{j+1}\right),
\end{aligned}
$$

onde $P_{j}(\mu)$ são as matrizes de Householder do vetor $w(\mu)$. 
Para evitar a possível perda de ortogonalidade dos vetores $q^{(j)}(\mu)$ e calcular a matriz $\delta_{k} J(\mu)$ com um custo computacional razoável, $J(\mu)$ é calculada pelo algoritmo de Lanczos 2.2 (com ortogonalização) e $\delta_{k} J(\mu)$ é obtida como no algoritmo 3.1. Isto leva ao algoritmo $\delta$-Lanczos com ortogonalização completa:

\section{ALGORITMO 3.2:}

Calcular $q^{1}(\mu)$, e $a_{1}(\mu), b_{j}(\mu), r^{j+1}(\mu), q^{j+1}(\mu), a_{j+1}(\mu), j=1,2, \ldots, n-1$ usando o algoritmo de Lanczos 2.2

Para $k=1, \ldots, n-1$

Calcular $\delta_{k} q^{(1)}(\mu)$

$$
\delta_{k} a_{1}(\mu):=2\left(\delta_{k} q^{(1)}(\mu)\right)^{T} \Lambda q^{(1)}(\mu)
$$

For $j=1, \ldots, n-1$

$$
\begin{aligned}
& \delta_{k} r^{(j+1)}(\mu):=\left(\Lambda-a_{j}(\mu) I\right) \delta_{k} q^{(j)}(\mu)-\delta_{k} a_{j}(\mu) \delta_{k} q^{(j)}(\mu)-\delta_{k} b_{j-1}(\mu) q^{(j-1)}(\mu) \\
& -b_{j-1}(\mu) \delta_{k} q^{(j-1)}(\mu) \\
& \delta_{k} b_{j}(\mu):=\frac{\left(r^{(j+1)}(\mu)\right)^{T} \delta_{k} r^{(j+1)}(\mu)}{b_{j}(\mu)} \\
& \delta_{k} q^{(j+1)}(\mu):=\frac{b_{j}(\mu) \delta_{k} r^{(j+1)}(\mu)-\delta_{k} b_{j}(\mu) r^{(j+1)}(\mu)}{\left(b_{j}(\mu)\right)^{2}} \\
& \delta_{k} a_{j+1}(\mu):=2\left(\delta_{k} q^{(j+1)}(\mu)\right)^{T} \Lambda q^{(j+1)}(\mu)
\end{aligned}
$$

O algoritmo $\delta$-Lanczos com ortogonalização completa requer $O\left(n^{3}\right)$ operações para calcular a matriz $J(\mu)$ e, como o algoritmo $3.1, O\left(n^{3}-n^{2}\right)$ operações para as matrizes $\delta_{k} J(\mu), k=$ $1,2, \ldots, n-1$. Assim o trabalho total é $O\left(2 n^{3}\right)$.

Observação: A última linha da matriz de autovetores $q^{(n)}(\mu)$ pode ser obtida a partir dos autovalores $\left(\lambda_{i}\right)_{1}^{n}$ e $\left(\mu_{i}\right)_{1}^{n-1}$ usando a Fórmula 2.8. Neste caso, as componentes do vetor $\delta_{k} q^{(n)}(\mu)$ são dadas por 


$$
\delta_{k} q_{i}^{(n)}(\mu)=\frac{1}{2} \frac{\prod_{j=1, j \neq k}^{n-1}\left(\mu_{j}-\lambda_{i}\right)}{\prod_{j=1, j \neq i}^{n-1}\left(\lambda_{j}-\lambda_{i}\right)}\left(\frac{\prod_{j=1, j \neq i}^{n-1}\left(\lambda_{j}-\lambda_{i}\right)}{\prod_{j=1}^{n-1}\left(\mu_{j}-\lambda_{i}\right)}\right)^{1 / 2} .
$$

para $i=1,2, \ldots n$, e $k=1,2, \ldots, n-1$.

\section{$3.3 \quad$ O método $\delta^{2}$-Lanczos}

$\mathrm{Na}$ Seção 3.4 são apresentados alguns resultados computacionais para demonstrar a eficiência, consistência, e precisão do método $\delta$-Lanczos. A precisão do método é confirmada numericamente usando o método de Newton para determinar um mínimo da função $G(\mu)$ de 3.39 , definida na próxima Seção. O uso de algum método de otimização pode requer o vetor gradiente e a matriz Hessiana da função. As derivadas de segunda ordem da matriz de Jacobi $J$ são calculadas usando uma versão de segunda ordem do método $\delta$-Lanczos. Este calcula as derivadas parciais da matriz $\delta_{k} J(\mu)$ com relação a cada variável $\mu_{l}, l=1,2, \ldots, n-1$, as quais são denotadas por $\delta_{l} \delta_{k} J(\mu), l=1,2, \ldots, n-1$.

Para cada $l=1,2, \ldots, n-1$, o vetor $\delta_{l} \delta_{k} q^{(1)}(\mu), k=1,2, \ldots, n-1$ é calculado primeiro, o qual denota a derivada parcial do vetor $\delta_{k} q^{(1)}(\mu)$ com relação à variavél $\mu_{l}$. Diferenciando a eq 3.8 com relação a $\mu_{l}$, obtém-se

$$
\delta_{l} \delta_{k} a_{1}(\mu)=2\left(\left(\delta_{l} \delta_{k} q^{(1)}(\mu)\right)^{T} \Lambda q^{(1)}(\mu)+\left(\delta_{k} q^{(1)}(\mu)\right)^{T} \Lambda \delta_{l} q^{(1)}(\mu)\right)
$$

Uma vez obtida $\delta_{l} \delta_{k} a_{1}(\mu)$, pode calcular-se $\delta_{l} \delta_{k} r^{(2)}(\mu)$ diferenciando a eq 3.12. Isto é,

$$
\begin{aligned}
\delta_{l} \delta_{k} r^{(2)}(\mu)= & \left(\Lambda-a_{1}(\mu) I\right) \delta_{l} \delta_{k} q^{(1)}(\mu)-\delta_{l} a_{1}(\mu) \delta_{k} q^{(1)}(\mu) \\
& -\delta_{l} \delta_{k} a_{1}(\mu) q^{(1)}(\mu)-\delta_{k} a_{1}(\mu) \delta_{l} q^{(1)}(\mu) .
\end{aligned}
$$

Agora, diferenciando as eqs 3.13 e 3.14 obtém-se:

$$
\begin{aligned}
\delta_{l} \delta_{k} b_{1}(\mu)= & \left\{b_{1}(\mu)\left(\left(\delta_{l} r^{(2)}(\mu)\right)^{T} \delta_{k} r^{(2)}(\mu)+\left(r^{(2)}(\mu)\right)^{T} \delta_{l} \delta_{k} r^{(2)}(\mu)\right)\right. \\
& \left.-\delta_{l} b_{1}(\mu)\left(r^{(2)}(\mu)\right)^{T} \delta_{k} r^{(2)}(\mu)\right\} /\left(b_{1}(\mu)\right)^{2}
\end{aligned}
$$




$$
\begin{aligned}
\delta_{l} \delta_{k} q^{(2)}(\mu)= & \left\{( b _ { 1 } ( \mu ) ) ^ { 2 } \left(\delta_{l} b_{1}(\mu) \delta_{k} r^{(2)}(\mu)+b_{1}(\mu) \delta_{l} \delta_{k} r^{(2)}(\mu)\right.\right. \\
& \left.-\delta_{l} \delta_{k} b_{1}(\mu) r^{(2)}(\mu)+\delta_{k} b_{1}(\mu) \delta_{l} r^{(2)}(\mu)\right) \\
& -2 b_{1}(\mu) \delta_{l} b_{1}(\mu)\left(b_{1}(\mu) \delta_{k} r^{(2)}(\mu)\right. \\
& \left.\left.-\delta_{k} b_{1}(\mu) r^{(2)}(\mu)\right)\right\} /\left(b_{1}(\mu)\right)^{4}
\end{aligned}
$$

Depois que $\delta_{l} \delta_{k} a_{1}(\mu), \delta_{l} \delta_{k} b_{1}(\mu)$ foram determinadas, a diferenciação, com relação a $\mu_{l}$, das eqs $3.17,3.21,3.22,3.23$ permite calcular, para cada $j=2,3, \ldots, n-1$ :

$$
\begin{aligned}
& \delta_{l} \delta_{k} a_{j}(\mu)=2\left(\left(\delta_{l} \delta_{k} q^{(j)}(\mu)\right)^{T} \Lambda q^{(j)}(\mu)+\left(\delta_{k} q^{(j)}(\mu)\right)^{T} \Lambda \delta_{l} q^{(j)}(\mu)\right) \\
& \delta_{l} \delta_{k} r^{(j+1)}(\mu)=\left(\Lambda-a_{j}(\mu) I\right) \delta_{l} \delta_{k} q^{(j)}(\mu)-\delta_{l} a_{j}(\mu) \delta_{k} q^{(j)}(\mu) \\
&-\delta_{l} \delta_{k} a_{j}(\mu) q^{(j)}(\mu)-\delta_{k} a_{j}(\mu) \delta_{l} q^{(j)}(\mu) \\
&-\delta_{l} \delta_{k} b_{j-1}(\mu) q^{(j-1)}(\mu)-\delta_{k} b_{j-1}(\mu) \delta_{l} q^{(j-1)}(\mu) \\
&-\delta_{l} b_{j-1}(\mu) \delta_{k} q^{(j-1)}(\mu)-b_{j-1}(\mu) \delta_{l} \delta_{k} q^{(j-1)}(\mu), \\
& \delta_{l} \delta_{k} b_{j}(\mu)=\left\{b _ { j } ( \mu ) \left(\left(\delta_{l} r^{(j+1)}(\mu)\right)^{T} \delta_{k} r^{(j+1)}(\mu)\right.\right. \\
&\left.+\left(r^{(j+1)}(\mu)\right)^{T} \delta_{l} \delta_{k} r^{(j+1)}(\mu)\right) \\
&\left.-\delta_{l} b_{j}(\mu)\left(r^{(j+1)}(\mu)\right)^{T} \delta_{k} r^{(j+1)}(\mu)\right\} /\left(b_{j}(\mu)\right)^{2} \\
& \\
& \\
& \delta_{l} \delta_{k} q^{(j+1)}(\mu)=\left\{( b _ { j } ( \mu ) ) ^ { 2 } \left(\delta_{l} b_{j}(\mu) \delta_{k} r^{(j+1)}(\mu)+b_{j}(\mu) \delta_{l} \delta_{k} r^{(j+1)}(\mu)\right.\right. \\
&\left.-\delta_{l} \delta_{k} b_{j}(\mu) r^{(j+1)}(\mu)-\delta_{k} b_{j}(\mu) \delta_{l} r^{(j+1)}(\mu)\right) \\
&-2 b_{j}(\mu) \delta_{l} b_{j}(\mu)\left(b_{j}(\mu) \delta_{k} r^{(j+1)}(\mu)\right. \\
&\left.\left.-\delta_{k} b_{j}(\mu) r^{(j+1)}(\mu)\right)\right\} /\left(b_{j}(\mu)\right)^{4} .
\end{aligned}
$$

As relações anteriores podem ser resumidas no algoritmo $\delta^{2}$-Lanczos:

ALGORITMO 3.3:

Calcular $q^{1}(\mu), a_{1}(\mu), e b_{j}(\mu), r^{j+1}(\mu), q^{j+1}(\mu), a_{j+1}(\mu), j=1,2, \ldots, n-1$ usando 
o algoritmo de Lanczos 2.1

Calcular $\delta_{k} q^{1}(\mu), \delta_{k} a_{1}(\mu), e \delta_{k} b_{j}(\mu), \delta_{k} r^{j+1}(\mu), \delta_{k} q^{j+1}(\mu), \delta_{k} a_{j+1}(\mu)$, $j=1,2, \ldots, n-1$ usando o algoritmo $\delta$-Lanczos 3.2

Para cada $l=1, \ldots, n-1$

Para cada $k=1, \ldots, n-1$

Calcular $\delta_{l} \delta_{k} q^{(1)}(\mu), \delta_{l} \delta_{k} a_{1}(\mu):=2\left(\left(\delta_{l} \delta_{k} q^{(1)}(\mu)\right)^{T} \Lambda q^{(1)}(\mu)+\delta_{k} q^{(1)}(\mu)\right)^{T} \Lambda \delta_{l} q^{(1)}(\mu)$

Para cada $j=1, \ldots, n-1$

$$
\begin{aligned}
\delta_{l} \delta_{k} r^{(j+1)}(\mu)= & \left(\Lambda-a_{j}(\mu) I\right) \delta_{l} \delta_{k} q^{(j)}(\mu)-\delta_{l} a_{j}(\mu) \delta_{k} q^{(j)}(\mu) \\
& -\delta_{l} \delta_{k} a_{j}(\mu) q^{(j)}(\mu)-\delta_{k} a_{j}(\mu) \delta_{l} q^{(j)}(\mu) \\
& -\delta_{l} \delta_{k} b_{j-1}(\mu) q^{(j-1)}(\mu)-\delta_{k} b_{j-1}(\mu) \delta_{l} q^{(j-1)}(\mu) \\
& -\delta_{l} b_{j-1}(\mu) \delta_{k} q^{(j-1)}(\mu)-b_{j-1}(\mu) \delta_{l} \delta_{k} q^{(j-1)}(\mu) \\
\delta_{l} \delta_{k} b_{j}(\mu)=\left\{b _ { j } ( \mu ) \left(\left(\delta_{l} r^{(j+1)}(\mu)\right)^{T} \delta_{k} r^{(j+1)}(\mu)\right.\right. & \\
+ & \left.\left(r^{(j+1)}(\mu)\right)^{T} \delta_{l} \delta_{k} r^{(j+1)}(\mu)\right) \\
- & \left.\delta_{l} b_{j}(\mu)\left(r^{(j+1)}(\mu)\right)^{T} \delta_{k} r^{(j+1)}(\mu)\right\} /\left(b_{j}(\mu)\right)^{2} \\
\delta_{l} \delta_{k} q^{(j+1)}(\mu)= & \left\{( b _ { j } ( \mu ) ) ^ { 2 } \left(\delta_{l} b_{j}(\mu) \delta_{k} r^{(j+1)}(\mu)+b_{j}(\mu) \delta_{l} \delta_{k} r^{(j+1)}(\mu)\right.\right. \\
& \left.-\delta_{l} \delta_{k} b_{j}(\mu) r^{(j+1)}(\mu)-\delta_{k} b_{j}(\mu) \delta_{l} r^{(j+1)}(\mu)\right) \\
& -2 b_{j}(\mu) \delta_{l} b_{j}(\mu)\left(b_{j}(\mu) \delta_{k} r^{(j+1)}(\mu)\right. \\
& \left.\left.-\delta_{k} b_{j}(\mu) r^{(j+1)}(\mu)\right)\right\} /\left(b_{j}(\mu)\right)^{4} \\
\delta_{l} \delta_{k} a_{j+1}(\mu)= & 2\left(\left(\delta_{l} \delta_{k} q^{(j+1)}(\mu)\right)^{T} \Lambda q^{(j+1)}(\mu)\right. \\
+ & \left.\left(\delta_{k} q^{(j+1)}(\mu)\right)^{T} \Lambda \delta_{l} q^{(j+1)}(\mu)\right)
\end{aligned}
$$

Observação:As derivadas de segunda ordem do vetor $q^{(n)}(\mu)$ são obtidas diferenciando as eqs 3.26 com relação as variáveis $\mu_{l}, l=1,2, \ldots, n-1$ : 
Para $i=1,2, \ldots n$,

$$
\begin{aligned}
\delta_{l} \delta_{k} q_{i}^{(n)}(\mu)= & \frac{1}{2} \prod_{j=1, j \neq l, k}^{n-1}\left(\mu_{j}-\lambda_{i}\right)\left\{\frac{1}{\prod_{j=1, j \neq i}^{n}\left(\lambda_{j}-\lambda_{i}\right)}\left(\frac{\prod_{j=1, j \neq i}^{n}\left(\lambda_{j}-\lambda_{i}\right)}{\prod_{j=1}^{n-1}\left(\mu_{j}-\lambda_{i}\right)}\right)^{1 / 2}-\right. \\
& \left.\frac{1}{2} \frac{1}{\prod_{j=1}^{n-1}\left(\mu_{j}-\lambda_{i}\right)}\left(\frac{\prod_{j=1}^{n-1}\left(\mu_{j}-\lambda_{i}\right)}{\prod_{j=1, j \neq i}^{n}\left(\lambda_{j}-\lambda_{i}\right)}\right)^{1 / 2}\right\}
\end{aligned}
$$

$k=1,2, \ldots, n-1$, com $l \neq k, \mathrm{e}$

$$
\delta_{l} \delta_{k} q_{i}^{(n)}(\mu)=-\frac{1}{4}\left(\frac{1}{\left(\mu_{l}-\lambda_{i}\right)}\right)^{2}\left(\frac{\prod_{j=1}^{n-1}\left(\mu_{j}-\lambda_{i}\right)}{\prod_{j=1, j \neq i}^{n}\left(\lambda_{j}-\lambda_{i}\right)}\right)^{1 / 2}
$$

$k=l=1,2, \ldots, n-1$.

\subsection{Resultados Computacionais}

Nesta Seção são apresentados alguns exemplos numéricos para demonstrar a eficiência e precisão do método $\delta$-Lanczos. No primeiro exemplo são calculadas e apresentadas graficamente as derivadas da matriz de Jacobi de ordem $n=10$, cujos elementos diagonais e co-diagonais são $a_{i}=10.0, b_{i}=3.0$, respectivamente. No segundo exemplo, é mostrado que em alguns casos o algoritmo de Lanczos com e sem ortogonalização são equivalentes. Isto mostra que, em certos casos, pode usar-se indistintamente ambos algoritmos. Logo depois, é demonstrada a consistência do método de $\delta$-Lanczos para as matrizes de ordem $n=20$ e 160 com os mesmos elementos da matriz do primeiro exemplo. $\mathrm{O}$ método é considerado consistente se os elementos da matriz $\delta_{k} J(\mu)$ calculados desde $\delta_{k} a_{1}, \delta_{k} b_{1}$ até os últimos elementos $\delta_{k} a_{n}, \delta_{k} b_{n-1}$ são iguais (em precisão de máquina) quando são calculados no sentido oposto. No seguinte exemplo, é usado o método de Newton para determinar um mínimo da função $G(\mu)$ de 3.39 , onde o vetor gradiente e a matriz Hessiana de $G(\mu)$ são calculados usando o método $\delta^{2}$-Lanczos (Ver Cap 5). A solução converge quadraticamente demonstrando que o método $\delta$-Lanczos calcula a derivada exata de $J(\mu)$. Finalmente, são usadas as matrizes de ordem $n=20$ e 160 com um elemento co-diagonal 
pequeno para mostrar a robustez do algoritmo $\delta$-Lanczos quando é comparado com o método de diferenças finitas.

\subsubsection{Gráficos das derivadas calculadas por $\delta$-Lanczos}

Considerar uma matriz de Jacobi de ordem $n=10$. Cada $a_{i}$ é igual a 10.0 e cada $b_{i}$ é igual a 3.0. Esta escolha proporciona o espectro $\left(\lambda_{i}\right)_{1}^{n}$ e $\left(\mu_{i}\right)_{1}^{n-1}$ correspondente aos autovalores da matriz de Jacobi e da submatriz principal superior, respectivamente. As componentes dos vetores $q^{(n)}(\mu)$ são calculadas das eqs 2.8 , e as dos vetores $\delta_{k} q^{(n)}(\mu), k=1,2, \ldots, n-1$ pelas eqs 3.26. Então o algoritmo $\delta$-Lanczos 3.2 (com ortogonalização) é aplicado para recuperar as matrizes $\delta_{k} J(\mu)$, $k=1,2, \ldots, n-1$.

Os erros relativos dos elementos diagonais e co-diagonais entre a matriz exata e a obtida depois de aplicar o algoritmo $\delta$-Lanczos 3.2 (com ortogonalização) são $e_{a}=1.200817 e-13, e_{b}=$ $7.776495 e-14$, respectivamente.

A Figura 3.1 apresenta os gráficos das derivadas dos elementos diagonais $\left(\delta_{k} a_{i}\right)_{i=1}^{n}$ e codiagonais $\left(\delta_{k} b_{i}\right)_{i=1}^{n-1}$, para cada $k=1,2, \ldots, n-1$. Nesta aplicação, os gráficos mostram que

$$
\delta_{1} a_{i}=\delta_{9} a_{i}, i=1,2, \ldots, n,
$$

e

$$
\delta_{1} b_{i}=-\delta_{9} b_{i}, i=1,2, \ldots, n-1 .
$$

São obtidos resultados semelhantes com o resto dos gráficos. Além disso, todas as figuras mostram, para cada $k$, que $\delta_{k} a_{i}=-\delta_{k} a_{n-(i-1)}, i=1,2, \ldots, n / 2$, e $\delta_{k} b_{i}=-\delta_{k} b_{n-i}, i=$ $1,2, \ldots,(n / 2)-1$. Os gráficos sugerem que as derivadas, para o tipo de matriz de Jacobi considerada, tem um comportamento de autovetor.

\subsubsection{Comparação dos algoritmos com e sem ortogonalização}

Nesta Seção são ilustrados alguns resultados numéricos, os quais mostram que, em certos casos, a re-ortogonalização do método $\delta$-Lanczos não é necessária. Primeiro é considerada uma matriz de ordem $n=20$ com $a_{i}=10.0, b_{i}=3.0$ exceto $b_{5}=1.0 e-05$. Para comparar os algoritmos são definidos os erros $\hat{e}_{a}=\frac{\left\|a^{r}-a\right\|_{\infty}}{\left\|a^{r}\right\|_{\infty}}, \hat{e}_{b}=\frac{\left\|b^{r}-b\right\|_{\infty}}{\left\|b^{r}\right\|_{\infty}}$. Aqui, as componentes dos vetores $a^{r}, b^{r}$ são os elementos da matriz obtida pelo algoritmo de Lanczos com ortogonalização e $a, b$ são sem ortogonalização. Para a matriz anterior tem-se, $\hat{e}_{a}=1.517101 e-10$, e $\hat{e}_{b}=2.901548 e-10$. 

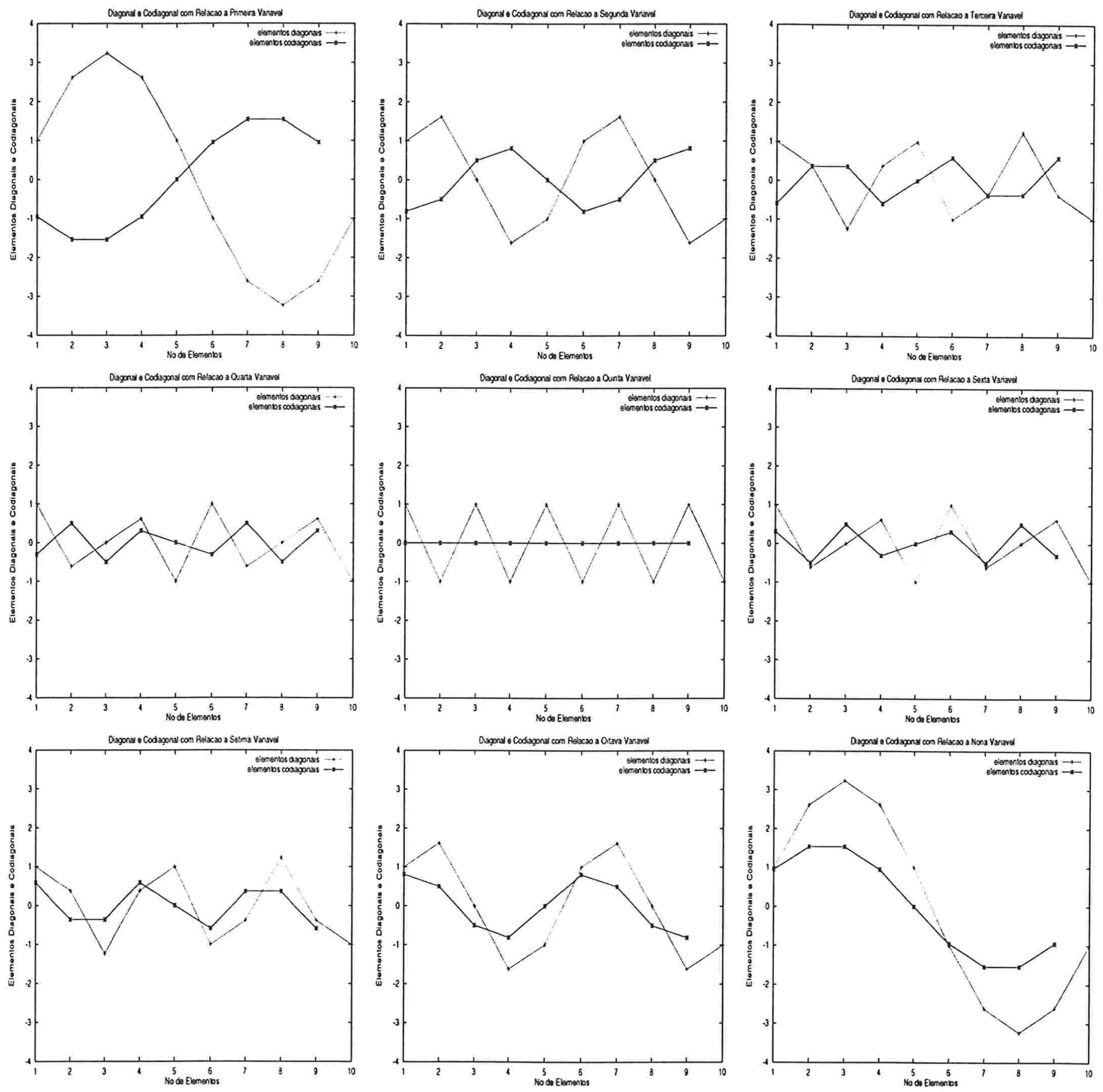

Figura 3.1: Gráficos das derivadas dos elementos diagonais e co-diagonais de uma matriz de Jacobi ordem $n=10$ com relação à primeira variável até a nona. 
Os algoritmos $\delta$-Lanczos (com e sem ortogonalização) são comparados definindo os erros $\delta_{k} \hat{e}_{a}=\frac{\left\|\delta_{k} a^{r}-\delta_{k} a\right\|_{\infty}}{\left\|\delta_{k} a^{r}\right\|_{\infty}}, \delta_{k} \hat{e}_{b}=\frac{\left\|\delta_{k} b^{r}-\delta_{k} b\right\|_{\infty}}{\left\|\delta_{k} b^{r}\right\|_{\infty}}$. Estes erros são ilustrados na Tabela 3.1, quando as derivadas da matriz anterior são calculadas com relação a $\mu_{k}, k=1,2,10,11,12$. Obtém-se resultados semelhantes para o resto das variáveis.

Se a dimensão da matriz é maior, os resultados são semelhantes ao caso de uma matriz de ordem $n=20$. Os resultados na Tabela 3.2, correspondente aos erros de uma matriz de ordem $n=160$, com $a_{i}=10.0, b_{i}=3.0$ exceto $b_{15}=1.0 e-04$. Para este exemplo os algoritmos levam aos erros $\hat{e}_{a}=2.287917 e-11$, e $\hat{e}_{b}=3.057232 e-11$.

Os resultados ilustrados nas Tabelas 3.1 e 3.2 mostram que ambos algoritmos levam a resultados semelhantes, embora algum elemento co-diagonal seja pequeno. Assim, a partir daqui os resultados apresentados são obtidos sem ortogonalização.

Tabela 3.1:

\begin{tabular}{lcc}
\hline \hline$k$ & $\delta_{k} \hat{e}_{a}$ & $\delta_{k} \hat{e}_{b}$ \\
\hline \hline 1 & $9.116094 \mathrm{e}-10$ & $6.473721 \mathrm{e}-10$ \\
2 & $1.757715 \mathrm{e}-09$ & $1.030581 \mathrm{e}-09$ \\
10 & $1.589851 \mathrm{e}-11$ & $5.244601 \mathrm{e}-09$ \\
11 & $2.955277 \mathrm{e}-10$ & $1.182479 \mathrm{e}-09$ \\
12 & $3.520908 \mathrm{e}-10$ & $9.681891 \mathrm{e}-10$ \\
\hline \hline
\end{tabular}

Tabela 3.2:

\begin{tabular}{ccc}
\hline \hline$k$ & $\delta_{k} \hat{e}_{a}$ & $\delta_{k} \hat{e}_{b}$ \\
\hline \hline 1 & $9.338647 \mathrm{e}-09$ & $9.894219 \mathrm{e}-09$ \\
2 & $2.143753 \mathrm{e}-09$ & $2.134142 \mathrm{e}-09$ \\
80 & $3.404998 \mathrm{e}-11$ & $1.659217 \mathrm{e}-08$ \\
81 & $9.115002 \mathrm{e}-11$ & $1.420093 \mathrm{e}-10$ \\
82 & $8.401080 \mathrm{e}-11$ & $1.852816 \mathrm{e}-10$ \\
\hline \hline
\end{tabular}

\subsubsection{Consistência do algoritmo $\delta$-Lanczos}

Para mostrar a consistência do algoritmo $\delta$-Lanczos, são consideradas as matrizes de ordem $n=$ 20 e 160 com $a_{i}=10.0, b_{i}=3.0$. Aqui, $\delta_{k} \hat{e}_{a}, \delta_{k} \hat{e}_{b}$ são definidos por $\delta_{k} \hat{e}_{a}=\frac{\left\|\delta_{k} a^{\uparrow}(\mu)-\delta_{k} a^{\downarrow}(\mu)\right\|_{\infty}}{\left\|\delta_{k} a^{\dagger}(\mu)\right\|_{\infty}}, \delta_{k} \hat{e}_{b}=$ 
$\frac{\left\|\delta_{k} b^{\uparrow}(\mu)-\delta_{k} b^{\downarrow}(\mu)\right\|_{\infty}}{\left\|\delta_{k} b^{\uparrow}(\mu)\right\|_{\infty}}$, onde as componentes de $\delta_{k} a^{\downarrow}$ e $\delta_{k} b^{\downarrow}$ são os elementos da matriz $\delta_{k} J(\mu)$ para ser calculados pelo algoritmo $\delta$-Lanczos a partir dos primeiros elementos $\delta_{k} a_{1}^{\downarrow}, \delta_{k} b_{1}^{\downarrow}$ até os últimos $\delta_{k} a_{n}^{\downarrow}, \delta_{k} b_{n-1}^{\downarrow}$, e $\delta_{k} a^{\uparrow}$ e $\delta_{k} b^{\uparrow}$ são no sentido oposto.

Na Tabela 3.3 são apresentados os erros $\delta_{k} \hat{e}_{a}, \delta_{k} \hat{e}_{b}$ para a matriz de ordem $n=20$. Neste caso, são calculadas as derivadas com relação a $\mu_{k}, k=1,2,10,11,12$. Estes resultados são semelhantes se a ordem da matriz é incrementada, embora os erros para os elementos extremos sejam maiores. Por exemplo, a Tabela 3.4 ilustra os erros da matriz de ordem $n=160$.

Tabela 3.3:

\begin{tabular}{lcc}
\hline \hline$k$ & $\delta_{k} \hat{e}_{a}$ & $\delta_{k} \hat{e}_{b}$ \\
\hline \hline 1 & $1.300071 \mathrm{e}-12$ & $6.100675 \mathrm{e}-13$ \\
2 & $4.001243 \mathrm{e}-13$ & $1.341043 \mathrm{e}-13$ \\
10 & $9.992007 \mathrm{e}-14$ & $2.164934 \mathrm{e}-14$ \\
11 & $5.007105 \mathrm{e}-14$ & $1.998401 \mathrm{e}-14$ \\
12 & $1.001421 \mathrm{e}-13$ & $2.003952 \mathrm{e}-14$ \\
\hline \hline
\end{tabular}

Tabela 3.4:

\begin{tabular}{ccc}
\hline \hline$k$ & $\delta_{k} \hat{e}_{a}$ & $\delta_{k} \hat{e}_{b}$ \\
\hline \hline 1 & $5.469971 \mathrm{e}-10$ & $2.810018 \mathrm{e}-10$ \\
2 & $2.589999 \mathrm{e}-10$ & $1.301003 \mathrm{e}-10$ \\
80 & $4.996003 \mathrm{e}-14$ & $1.088417 \mathrm{e}-13$ \\
81 & $2.000066 \mathrm{e}-13$ & $8.998357 \mathrm{e}-14$ \\
82 & $1.399991 \mathrm{e}-13$ & $6.99956 \mathrm{e}-14$ \\
\hline \hline
\end{tabular}




\subsubsection{Derivada numérica exata obtida pelo algoritmo $\delta$-Lanczos}

Aqui é usado o método de Newton para confirmar numericamente a precisão das derivadas calculadas pelo algoritmo $\delta$-Lanczos. O problema modelo é baseado na minimização da função

$$
G(\mu)=\sum_{j=1}^{n}\left(a_{j}(\mu)-\bar{a}_{j}\right)^{2}+\sum_{j=1}^{n-1}\left(b_{j}(\mu)-\bar{b}_{j}\right)^{2},
$$

sujeito a $\bar{\lambda}_{i}<\mu_{i}<\bar{\lambda}_{i+1}, i=1,2, \ldots, n-1$, onde $\bar{\lambda}_{i}$ são os autovalores da matriz de Jacobi $\bar{J}$ com elementos $\left(\bar{a}_{i}\right)_{1}^{n},\left(\bar{b}_{i}\right)_{1}^{n-1}$ dados na Tabela 3.5 , e $\left(a(\mu)_{i}\right)_{1}^{n},\left(b(\mu)_{i}\right)_{1}^{n-1}$ são os elementos da matriz de Jacobi $J(\mu)$ reconstruída a partir dos $\bar{\lambda}_{i}$ e $\mu_{i}$. A solução deste problema leva a um espectro intercalado $\bar{\mu}_{i}$ associado com $\bar{J}$, e neste ponto o gradiente de $G$ é zero. Aplicando o método de Newton para achar um zero de $G$, e se as derivadas são calculadas exatamente, a convergência a $\overline{\mu_{i}}$ será quadrática. Isto é confirmado pelos gráficos nas Figuras 3.2(a)-3.2(c).

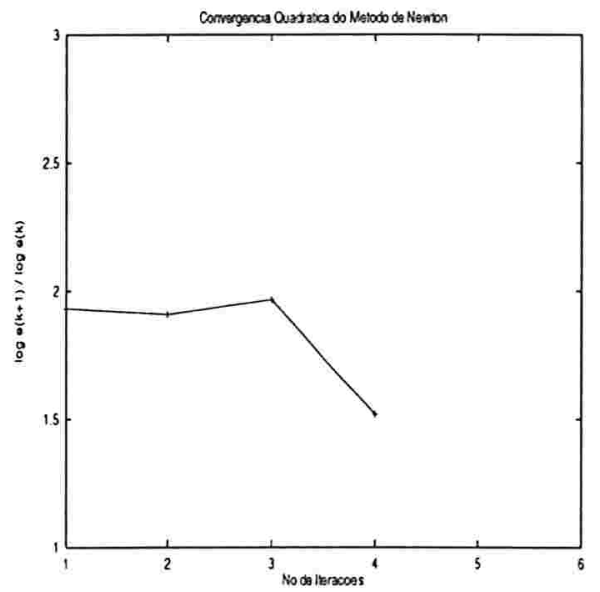

(a)

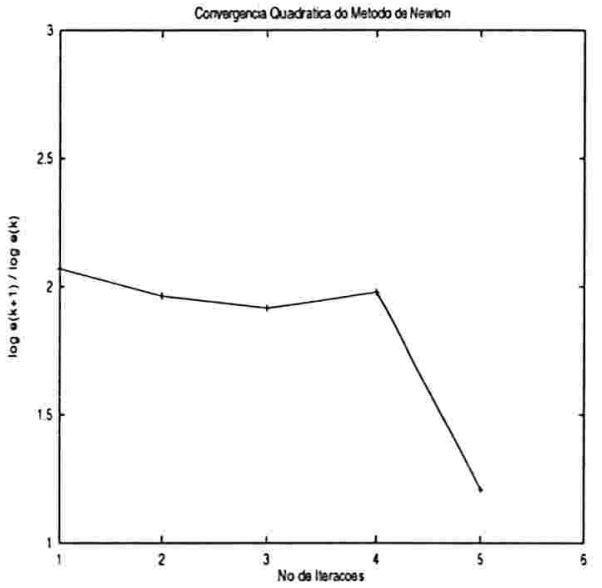

(b)

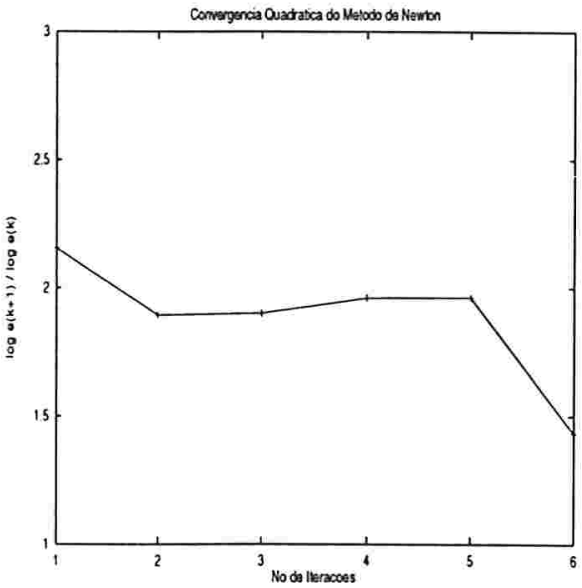

(c)

Figura 3.2: Gráficos dos $c_{j}=\log \left(e_{j+1}\right) / \log \left(e_{j}\right)$, onde $e_{j}$ denota o erro absoluto entre a solução exata e aproximada na j-ésima iteração do método de Newton para três valores iniciais diferentes.

Estes Figuras mostram os gráficos dos $c_{j}=\log \left(e_{j+1}\right) / \log \left(e_{j}\right)$, onde $e_{j}$ denota o erro absoluto entre a solução exata e aproximada na $j$-ésima iteração para três valores iniciais diferentes. Para o primeiro valor inicial, especificado na Tabela 3.5, a convergência é quadrática em 5 iterações, como mostra a Figura 3.2(a). Neste caso, para todo $j, c_{j} \simeq 2.0$ exceto $c_{4}$, já que $e_{5}$ alcança a precisão de máquina. Os valores iniciais listados nas últimas dos colunas da Tabela 3.5 levam confirmar numericamente que o algoritmo $\delta$-Lanczos calcula as derivadas exatas de uma matriz de Jacobi. 
Tabela 3.5:

\begin{tabular}{cccccccc}
\hline \hline$i$ & $\bar{\lambda}_{i}$ & $\mu_{i}^{\text {exato }}$ & $\bar{a}_{i}$ & $\bar{b}_{i}$ & $1^{\circ} \mu_{i}^{v i}$ & $2^{\circ} \mu_{i}^{v i}$ & $3^{\circ} \mu_{i}^{v i}$ \\
\hline \hline 1 & 2.300 & 4.100 & 16.919 & 3.986 & 4.200 & 4.000 & 4.200 \\
2 & 5.700 & 7.300 & 20.258 & 6.735 & 7.300 & 7.200 & 7.100 \\
3 & 9.500 & 10.90 & 18.743 & 6.981 & 10.900 & 10.800 & 10.700 \\
4 & 13.400 & 15.000 & 19.960 & 7.496 & 15.000 & 15.100 & 15.000 \\
5 & 16.700 & 18.100 & 18.093 & 8.164 & 18.100 & 18.000 & 18.000 \\
6 & 21.100 & 23.300 & 20.742 & 9.060 & 23.300 & 23.300 & 23.200 \\
7 & 25.400 & 27.700 & 20.711 & 8.709 & 27.700 & 27.600 & 27.700 \\
8 & 30.000 & 31.300 & 19.866 & 9.286 & 31.500 & 31.300 & 31.200 \\
9 & 33.300 & 35.800 & 18.208 & 12.412 & 35.800 & 35.800 & 35.700 \\
10 & 37.100 & & 21.000 & & & & \\
\hline \hline
\end{tabular}

\subsubsection{Comparação dos métodos $\delta$-Lanczos e diferenças finitas}

São considerados dois exemplos para comparar o método diferenças finitas com o método $\delta$ Lanczos. Primeiro, é considerada uma matriz de ordem $n=20$, cujos elementos são $a_{i}=10.0$, $b_{i}=3.0$ exceto $b_{5}=0.2$. Logo depois, a ordem da matriz é incrementada a $n=160$. Os valores $\delta_{3} \hat{e}_{a}, \delta_{3} \hat{e}_{b}$ indicam os erros dos elementos diagonais e co-diagonais da matriz $\delta_{3} J(\mu)$ calculada por $\delta$-Lanczos e diferenças finitas. Neste caso, é usado a aproximação por diferenças centradas de segunda ordem .

A dificuldade do esquema de diferenças finitas é a escolha do passo $\Delta \mu_{k}$. Por exemplo, as Tabelas 3.6 e 3.7 mostram os passos para determinar a derivada da matriz de Jacobi mencionada anteriormente, com relação a $\mu_{3}$. Quando $n=20$ o passo ótimo é $\Delta \mu_{3}=1.0 e-08$, enquanto para $n=160$ é $\Delta \mu_{3}=1.0 e-07$. Isto mostra que o passo $\Delta \mu_{k}$ depende da dimensão de $J$, e quando a ordem da matriz é maior os erros são maiores.

Por outro lado, se é desejado minimizar a função $G(\mu)$ de 3.39 computando as derivadas do vetor gradiente usando diferenças finitas, a escolha está restrita pela propriedade de intercalamento

$$
\lambda_{k}<\mu_{k}-\Delta \mu_{k}, \quad \mu_{k}+\Delta \mu_{k}<\lambda_{k+1} .
$$

Isto implica que para cada passo iterativo deve ser determinado o valor ótimo de $\Delta \mu_{k}$. Estas dificuldades ressaltam as vantagens do método $\delta$-Lanczos para o análise de sensibilidade das matrizes de Jacobi. 
Tabela 3.6:

\begin{tabular}{ccc}
\hline \hline$\Delta \mu_{3}$ & $\delta_{3} \hat{e}_{a}$ & $\delta_{3} \hat{e}_{b}$ \\
\hline \hline $1.0 \mathrm{e}-04$ & $8.922547 \mathrm{e}-02$ & $5.630937 \mathrm{e}-02$ \\
$1.0 \mathrm{e}-05$ & $8.196562 \mathrm{e}-04$ & $5.382393 \mathrm{e}-04$ \\
$1.0 \mathrm{e}-06$ & $8.190057 \mathrm{e}-06$ & $5.380117 \mathrm{e}-06$ \\
$1.0 \mathrm{e}-07$ & $8.457750 \mathrm{e}-08$ & $5.648974 \mathrm{e}-08$ \\
$1.0 \mathrm{e}-08$ & $9.100510 \mathrm{e}-09$ & $7.297199 \mathrm{e}-09$ \\
$1.0 \mathrm{e}-09$ & $9.515141 \mathrm{e}-08$ & $9.169585 \mathrm{e}-08$ \\
$1.0 \mathrm{e}-10$ & $1.705823 \mathrm{e}-07$ & $2.105213 \mathrm{e}-08$ \\
$1.0 \mathrm{e}-11$ & $1.937736 \mathrm{e}-06$ & $2.393996 \mathrm{e}-06$ \\
\hline \hline
\end{tabular}

Tabela 3.7:

\begin{tabular}{ccc}
\hline \hline$\Delta \mu_{3}$ & $\delta_{3} \hat{e}_{a}$ & $\delta_{3} \hat{e}_{b}$ \\
\hline \hline $1.0 \mathrm{e}-04$ & 0.796029 & 0.793665 \\
$1.0 \mathrm{e}-05$ & $4.381208 \mathrm{e}-03$ & $4.370754 \mathrm{e}-03$ \\
$1.0 \mathrm{e}-06$ & $4.361880 \mathrm{e}-05$ & $4.351758 \mathrm{e}-05$ \\
$1.0 \mathrm{e}-07$ & $4.389864 \mathrm{e}-07$ & $4.368720 \mathrm{e}-07$ \\
$1.0 \mathrm{e}-08$ & $1.453702 \mathrm{e}-06$ & $1.689290 \mathrm{e}-06$ \\
$1.0 \mathrm{e}-09$ & $4.166141 \mathrm{e}-06$ & $4.773031 \mathrm{e}-06$ \\
$1.0 \mathrm{e}-10$ & $5.595735 \mathrm{e}-05$ & $1.095942 \mathrm{e}-05$ \\
$1.0 \mathrm{e}-11$ & $6.262415 \mathrm{e}-03$ & $1.260549 \mathrm{e}-02$ \\
\hline \hline
\end{tabular}




\section{Capítulo 4}

\section{Análise de Sensibilidade de Sistemas Massa-Mola}

Para resolver o problema variacional 3.2- 3.3 é necessário fazer um estudo prévio da sensibilidade das matrizes massa e rigidez de um sistema $(M, K)$, o qual é precedida da análise de sensibildade de uma matriz de Jacobi, discutido no Capítulo anterior. O gradiente da função

$$
F(\mu)=\sum_{l=1}^{n}\left(m_{l}(\mu)-\bar{m}_{l}\right)^{2}+\sum_{l=1}^{n}\left(k_{l}(\mu)-\bar{k}_{l}\right)^{2}
$$

é dado por

$$
\nabla F(\mu)=\left(\frac{\partial F(\mu)}{\partial \mu_{1}}, \frac{\partial F(\mu)}{\partial \mu_{2}}, \ldots, \frac{\partial F(\mu)}{\partial \mu_{n-1}}\right)
$$

onde

$$
\frac{\partial F(\mu)}{\partial \mu_{j}}=2\left(\sum_{l=1}^{n}\left(m_{l}(\mu)-\bar{m}_{l}\right) \frac{\partial m_{l}(\mu)}{\partial \mu_{j}}+\sum_{l=1}^{n}\left(k_{l}(\mu)-\bar{k}_{l}\right) \frac{\partial k_{l}(\mu)}{\partial \mu_{j}}\right),
$$

com $j=1,2, \ldots, n-1$.

Consequentemente com a notação do Cap 3, as derivadas parciais $\frac{\partial m_{l}(\mu)}{\partial \mu_{j}} \mathrm{e} \frac{\partial k_{l}(\mu)}{\partial \mu_{j}}$ serão denotadas por $\delta_{j} m_{l}(\mu)$ e $\delta_{j} k_{l}(\mu)$, respectivamente. Estes elementos formam as respectivas matrizes $\delta_{j} M(\mu) \equiv \frac{\partial M(\mu)}{\partial \mu_{j}}$ e $\delta_{j} K(\mu) \equiv \frac{\partial K(\mu)}{\partial \mu_{j}}$.

Nesta Seção é apresentado um método de reconstrução das matrizes $\delta_{j} M(\mu)$ e $\delta_{j} K(\mu)$. As derivadas calculadas são numericamente exatas exceto por erros de arredondamento. A reconstrução é precedida do algoritmo 3.1 (ou 3.2) para obter as matrizes $\left(\delta_{j} J(\mu)\right)_{1}^{n-1}$. Isto significa 
que a mesma informação espectral é necessária para obter as sensibilidades das matrizes massa e rigidez. Além disso, são obtidas as derivadas de segunda ordem de $M(\mu)$ e $K(\mu)$, as quais serão necessárias na solução do problema variacional. Após a análise de sensibilidade são apresentados exemplos numéricos para demonstrar a precisão e eficiência do método. O método de diferenças finitas, apesar de suas limitações, é usado como referência para nosso método.

\subsection{Análise de sensibilidade das matrizes $M(\mu)$ e $K(\mu)$}

Nesta Seção será descrito um método para calcular as sensibilidades das matrizes $M(\mu)$ e $K(\mu)$ com relação ao espectro intercalado $\mu=\left(\mu_{1}, \mu_{2}, \ldots, \mu_{n-1}\right)$. Este método permite calcular as derivadas de primeira e segunda ordem numericamente exatas com relação a cada $\mu_{j}$.

Os espectros $\left(\lambda_{l}\right)_{1}^{n},\left(\mu_{l}\right)_{1}^{n-1}$ e a massa total $m_{T}$ do sistema são os dados para calcular as matrizes $\delta_{j} M(\mu), \delta_{j} K(\mu)$, com $j=1,2, \ldots n-1$. No Cap 2 um algoritmo para calcular as matrizes $M(\mu)$ e $K(\mu)$ a partir dos autovalores e da massa total foi descrito. Este algoritmo reconstrói primeiro uma matriz de Jacobi $J(\mu)$, e depois as massas e constantes de rigidez. Para delinear o método que calcula as matrizes $\delta_{j} M(\mu), \delta_{j} K(\mu)$ suponha que a matriz $J(\mu)$ foi obtida pelo algoritmo 2.1 (ou 2.2). Assim, o vetor $y(\mu)=\left(y_{1}(\mu), y_{2}(\mu), \ldots, y_{n}(\mu)\right)^{T} \neq 0$ é a solução do sistema

$$
J(\mu) y(\mu)=e_{1}
$$

com $e_{1}=(1,0, \ldots, 0)^{T}$. Diferenciando a equação anterior com relação a $\mu_{j}$, obtém-se

$$
\delta_{j} J(\mu) y(\mu)+J(\mu) \delta_{j} y(\mu)=0
$$

ou, equivalentemente,

$$
J(\mu) \delta_{j} y(\mu)=-\delta_{j} J(\mu) y(\mu) .
$$

Resolvendo o sistema 4.2 conhece-se o vetor $\delta_{j} y(\mu)=\left(\delta_{j} y_{1}(\mu), \delta_{j} y_{2}(\mu), \ldots, \delta_{j} y_{n}(\mu)\right)^{T}$, onde a matriz $\delta_{j} J(\mu)$ é calculada pelo algoritmo 3.1 (ou 3.2).

Por outro lado, a última massa pode ser calculada pela relação

$$
m_{n}(\mu)=\frac{m_{T}}{1+\sum_{l=1}^{n-1}\left(\frac{y_{l}(\mu)}{y_{n}(\mu)}\right)^{2}}
$$


onde $m_{T}$, a princípio, é considerada constante. A derivada da $n$-ésima massa é calculada diferenciando a relação anterior, isto é,

$$
\delta_{j} m_{n}(\mu)=\frac{-2 m_{T} \sum_{l=1}^{n-1}\left(\frac{y_{l}(\mu)}{y_{n}^{3}(\mu)}\left(\delta_{j} y_{l}(\mu) y_{n}(\mu)-y_{l}(\mu) \delta_{j} y_{n}(\mu)\right)\right)}{\left[1+\sum_{l=1}^{n-1}\left(\frac{y_{l}(\mu)}{y_{n}(\mu)}\right)^{2}\right]^{2}} .
$$

Agora diferenciando o resto das massas, dadas pela relação

$$
m_{l}(\mu)=m_{n}(\mu)\left(\frac{y_{l}(\mu)}{y_{n}(\mu)}\right)^{2}, l=1,2, \ldots, n-1,
$$

obtém-se

$\delta_{j} m_{l}(\mu)=\delta_{j} m_{n}(\mu)\left(\frac{y_{l}(\mu)}{y_{n}(\mu)}\right)^{2}+2 m_{n}(\mu)\left(\frac{y_{l}(\mu)}{y_{n}^{3}(\mu)}\right)\left(\delta_{j} y_{l}(\mu) y_{n}(\mu)-y_{l}(\mu) \delta_{j} y_{n}(\mu)\right), l=1,2, \ldots, n-1$

Desta maneira, conhecidas todas as $\delta_{j} m_{l}(\mu)$, a sensibilidade da matriz massa é completamente determinada. Para obter $\delta_{j} K(\mu)$ considera-se a relação

$$
K(\mu)=\left(B^{T}(\mu)\right)^{-1} J(\mu) B^{-1}(\mu),
$$

onde $B(\mu)=B^{T}(\mu)=\operatorname{diag}\left\{m_{1}^{1 / 2}(\mu), m_{2}^{1 / 2}(\mu), \ldots, m_{n}^{1 / 2}(\mu)\right\}$. Daqui, pode-se verificar que

$$
k_{l}(\mu)= \begin{cases}m_{1}(\mu) a_{n}(\mu)-k_{2}(\mu) & , \text { se } l=1 \\ b_{n-l+1}(\mu) \sqrt{m_{l-1}(\mu) m_{l}(\mu)} & , \text { se } l=2,3, \ldots, n,\end{cases}
$$

onde $a_{n}(\mu), b_{n-l+1}(\mu)$ são elementos da matriz $J(\mu)$. Portanto, as sensibilidades das constantes de rigidez podem ser calculadas diferenciando a eq 4.5 , isto é,

$$
\delta_{j} k_{l}(\mu)= \begin{cases}\delta_{j} m_{1}(\mu) a_{n}(\mu)+m_{1}(\mu) \delta_{j} a_{n}(\mu)-\delta_{j} k_{2}(\mu) & , \text { se } l=1 \\ \delta_{j} b_{n-l+1}(\mu) \sqrt{m_{l-1}(\mu) m_{l}(\mu)}+\frac{b_{n-l+1}(\mu)}{2 \sqrt{m_{l-1}(\mu) m_{l}(\mu)}}\left(\delta_{j} m_{l-1}(\mu) m_{l}(\mu)\right. & \\ \left.+m_{l-1}(\mu) \delta_{j} m_{l}(\mu)\right) & \text {, se } l=2,3, \ldots, n .\end{cases}
$$


As eqs $4.3,4.4$, e 4.6 determinam as derivadas de primeira ordem das matrizes massa e rigidez. Isto é resumido no seguinte:

ALGORITMO 4.1: Dados os autovalores $\left(\lambda_{l}\right)_{1}^{n}$ e $\left(\mu_{l}\right)_{1}^{n-1}$ correspondentes aos sistemas $(M, K)$ e modificado $(\widetilde{M}, \widetilde{K})$, respectivamente, e a massa total $m_{T}$ de $(M, K)$, este produz as matrizes $\delta_{j} M(\mu)$ e $\delta_{j} K(\mu)$, para cada $j=1,2, \ldots n-1$ :

Calcular $\left(a_{l}(\mu)\right)_{l=1}^{n}$, e $\left(b_{l}(\mu)\right)_{l=1}^{n-1}$ da matriz $J(\mu)$ usando o algoritmo de Lanczos 2.1

Determinar $\left(y_{l}(\mu)\right)_{l=1}^{n}$ resolvendo o sistema $J(\mu) y(\mu)=e_{1}$

$$
\begin{aligned}
\text { Calcular } m_{n}(\mu) & =\frac{m_{T}}{1+\sum_{l=1}^{n-1}\left(\frac{y_{l}(\mu)}{y_{n}(\mu)}\right)^{2}} \\
m_{l}(\mu) & =m_{n}(\mu)\left(\frac{y_{l}(\mu)}{y_{n}(\mu)}\right)^{2}, l=1, \ldots, n-1 \\
k_{l}(\mu) & =b_{n-l+1}(\mu) \sqrt{m_{l-1}(\mu) m_{l}(\mu)}, l=2, \ldots, n \\
k_{1}(\mu) & =m_{1}(\mu) a_{n}(\mu)-k_{2}(\mu) \\
\text { Para } j & =1, \ldots, n-1
\end{aligned}
$$

Calcular $\left(\delta_{j} a_{l}(\mu)\right)_{l=1}^{n}$, e $\left(\delta_{j} b_{l}(\mu)\right)_{l=1}^{n-1}$ da matriz $\delta_{j} J(\mu)$ usando o algoritmo $\delta$-Lanczos 3.1

Determinar $\left(\delta_{j} y_{l}(\mu)\right)_{l=1}^{n}$ resolvendo o sistema $J(\mu) \delta_{j} y(\mu)=-\delta_{j} J(\mu) y(\mu)$

$$
\begin{gathered}
\text { Calcular } \delta_{j} m_{n}(\mu)=\frac{-2 m_{T} \sum_{l=1}^{n-1}\left(\frac{y_{l}(\mu)}{y_{n}^{3}(\mu)}\left(\delta_{j} y_{l}(\mu) y_{n}(\mu)-y_{l}(\mu) \delta_{j} y_{n}(\mu)\right)\right)}{\left[1+\sum_{l=1}^{n-1}\left(\frac{y_{l}(\mu)}{y_{n}(\mu)}\right)^{2}\right]^{2}} \\
\delta_{j} m_{l}(\mu)=\delta_{j} m_{n}(\mu)\left(\frac{y_{l}(\mu)}{y_{n}(\mu)}\right)^{2}+2 m_{n}(\mu)\left(\frac{y_{l}(\mu)}{y_{n}^{3}(\mu)}\right)\left(\delta_{j} y_{l}(\mu) y_{n}(\mu)-y_{l}(\mu) \delta_{j} y_{n}(\mu)\right), \\
l=1,2, \ldots, n-1 \\
\delta_{j} k_{l}(\mu)=\delta_{j} b_{n-l+1}(\mu) \sqrt{m_{l-1}(\mu) m_{l}(\mu)}+\frac{b_{n-l+1}(\mu)}{2 \sqrt{m_{l-1}(\mu) m_{l}(\mu)}}\left(\delta_{j} m_{l-1}(\mu) m_{l}(\mu)+\right.
\end{gathered}
$$




$$
\begin{aligned}
& \left.m_{l-1}(\mu) \delta_{j} m_{l}(\mu)\right), l=2, \ldots, n \\
& \delta_{j} k_{1}(\mu)=\delta_{j} m_{1}(\mu) a_{n}(\mu)+m_{1}(\mu) \delta_{j} a_{n(\mu)}-\delta_{j} k_{2}(\mu) .
\end{aligned}
$$

$\mathrm{O}$ algoritmo 4.1 pode ser dividido em duas partes; primeiro, determinar a matriz de Jacobi $J(\mu)$ e suas derivadas, e logo, as massas e constantes de rigidez relativas a $J(\mu)$ com suas correspondentes derivadas, o qual tem um custo computacional de $O\left(2 n^{3}\right)$ operações (usando o algoritmo de Lanczos com ortogonalização). Na segunda parte deve-se resolver uma vez o sistema tridiagonal $J(\mu) y(\mu)=e_{1}$ com um custo de $O(n)$ operaçòes, e determinar as massas e constantes de rigidez que requer um esforço computacional de ordem $O(n)$, incrementando o custo a $O\left(2\left(n^{3}+n\right)\right)$ operações. Além disso, para calcular as derivadas de $M(\mu)$ e $K(\mu)$ devem resolver-se $(n-1)$ vezes o sistema tridiagonal $J \delta_{j} y=-\delta_{j} J y$ e calcular as matrizes $\delta_{j} M(\mu)$ e $\delta_{j} K(\mu)$ com un custo de $O\left(n^{2}\right)$ operações. Assim, o trabalho total do algoritmo 4.1 é aproximadamente da $O\left(2 n^{3}+n^{2}+n\right)$ operações.

Uma vez determinadas as derivadas de primeira ordem das matrizes $M(\mu)$ e $K(\mu)$, as derivadas de segunda ordem são obtidas de uma maneira semelhante. As derivadas mistas $\frac{\partial M(\mu)}{\partial \mu_{i} \partial \mu_{j}} \mathrm{e}$ $\frac{\partial K(\mu)}{\partial \mu_{i} \partial \mu_{j}}$ serão denotadas por $\delta_{i} \delta_{j} M(\mu)$ e $\delta_{i} \delta_{j} K(\mu)$, respectivamente. Os elementos de $\delta_{i} \delta_{j} M(\mu)$ e $\delta_{i} \delta_{j} K(\mu)$ são $\delta_{i} \delta_{j} m_{l}(\mu)$ e $\delta_{i} \delta_{j} k_{l}(\mu)$, com $l=1,2, \ldots, n$. Se a eq 4.2 e diferenciada com relação a $\mu_{i}$ e os termos são reorganizados, obtém-se

$$
J(\mu) \delta_{i} \delta_{j} y(\mu)=-\left(\delta_{i} \delta_{j} J(\mu) y(\mu)+\delta_{i} J(\mu) \delta_{j} y(\mu)+\delta_{j} J(\mu) \delta_{i} y(\mu)\right),
$$

onde as matrizes $\delta_{j} J(\mu)$, e $\delta_{i} \delta_{j} J(\mu)$ podem ser obtidas do algoritmo 3.3. Assim, a solução do sistema 4.7 permite calcular as derivadas de segunda ordem das massas e constantes de rigidez, isto é, determinado o vetor $\delta_{i} \delta_{j} y(\mu)$ de 4.7 e diferenciando a eq 4.3 com relação a $\mu_{i}$, obtém-se

$$
\begin{aligned}
\delta_{i} \delta_{j} m_{n}(\mu)= & {\left[\left(1+\sum_{l=1}^{n-1}\left(\frac{y_{l}(\mu)}{y_{n}(\mu)}\right)^{2}\right)^{2}\right.} \\
& \left(-2 m_{T} \sum_{l=1}^{n-1}\left\{\frac{\left[\delta_{j} y_{l}(\mu) y_{n}^{3}(\mu)-3 y_{l}(\mu) y_{n}^{2}(\mu) \delta_{i} y_{n}(\mu)\right]\left[\delta_{j} y_{l}(\mu) y_{n}(\mu)-y_{l}(\mu) \delta_{j} y_{n}\right]}{y_{n}^{6}(\mu)}+\right.\right. \\
& \left.\left.+\left[\delta_{i} \delta_{j} y_{l}(\mu) y_{n}(\mu)+\delta_{j} y_{l}(\mu) \delta_{i} y_{n}(\mu)-\delta_{i} \delta_{j} y_{n}(\mu) y_{l}(\mu)-\delta_{j} y_{n}(\mu) \delta_{i} y_{l}(\mu)\right]\left(\frac{y_{l}(\mu)}{y_{n}^{3}(\mu)}\right)\right\}\right)+ \\
& +\left(8 m_{T} \sum_{l=1}^{n-1} \frac{y_{l}(\mu)}{y_{n}^{3}(\mu)}\left[\delta_{j} y_{l}(\mu) y_{n}(\mu)-y_{l}(\mu) \delta_{j} y_{n}(\mu)\right]\right)\left(1+\sum_{l=1}^{n-1}\left(\frac{y_{l}(\mu)}{y_{n}(\mu)}\right)^{2}\right)
\end{aligned}
$$




$$
\left.\left(\sum_{l=1}^{n-1} \frac{y_{l}(\mu)}{y_{n}^{3}(\mu)}\left[\delta_{i} y_{l}(\mu) y_{n}(\mu)-y_{l}(\mu) \delta_{i} y_{n}(\mu)\right]\right)\right] /\left[1+\sum_{l=1}^{n-1}\left(\frac{y_{l}(\mu)}{y_{n}(\mu)}\right)^{2}\right]^{4}
$$

Obtido $\delta_{i} \delta_{j} m_{n}(\mu)$, são calculadas $\delta_{i} \delta_{j} m_{l}(\mu), l=1,2, \ldots, n-1$ diferenciando a eq 4.4 , isto é,

$$
\begin{aligned}
\delta_{i} \delta_{j} m_{l}(\mu)= & \delta_{i} \delta_{j} m_{n}(\mu)\left(\frac{y_{l}(\mu)}{y_{n}(\mu)}\right)^{2}+2 \delta_{j} m_{n}(\mu)\left(\frac{y_{l}(\mu)}{y_{n}^{3}(\mu)}\right)\left[\delta_{i} y_{l}(\mu) y_{n}(\mu)-y_{l}(\mu) \delta_{i} y_{n}(\mu)\right]+ \\
& +2 \delta_{i} m_{n}(\mu)\left(\frac{y_{l}(\mu)}{y_{n}^{3}(\mu)}\right)\left[\delta_{j} y_{l}(\mu) y_{n}(\mu)-y_{l}(\mu) \delta_{j} y_{n}(\mu)\right]+ \\
& +2 m_{n}(\mu)\left(\frac{\left(\delta_{j} y_{l}(\mu) y_{n}^{3}(\mu)-3 y_{l}(\mu) y_{n}^{2}(\mu) \delta_{i} y_{n}(\mu)\right)}{y_{n}^{6}(\mu)}\right)\left[\delta_{j} y_{l}(\mu) y_{n}(\mu)-y_{l}(\mu) \delta_{j} y_{n}(\mu)\right]+ \\
& 2 m_{n}(\mu)\left(\frac{y_{l}(\mu)}{y_{n}^{3}(\mu)}\right)\left[\delta_{i} \delta_{j} y_{l}(\mu) y_{n}(\mu)+\delta_{j} y_{l}(\mu) \delta_{i} y_{n}(\mu)-\delta_{i} \delta_{j} y_{n}(\mu) y_{l}(\mu)-\delta_{j} y_{n}(\mu) \delta_{i} y_{l}(\mu)\right]
\end{aligned}
$$

para $l=1,2, \ldots, n-1$. Para calcular as derivadas mistas das constantes de rigidez são consideradas as eqs 4.6 para ser diferenciadas. Portanto,

$$
\begin{aligned}
\delta_{i} \delta_{j} k_{l}(\mu)= & {\left[2 \left\{\left[\delta_{i} \delta_{j} m_{l-1}(\mu) m_{l}(\mu)+\delta_{j} m_{l-1}(\mu) \delta_{i} m_{l}(\mu)+\delta_{i} \delta_{j} m_{l}(\mu) m_{l-1}(\mu)+\right.\right.\right.} \\
& \left.+\delta_{j} m_{l}(\mu) \delta_{i} m_{l-1}(\mu)\right] b_{n-l+1}(\mu)+\left[\delta_{j} m_{l-1}(\mu) m_{l}(\mu)+\right. \\
& \left.\left.+\delta_{j} m_{l}(\mu) m_{l-1}(\mu)\right] \delta_{i} b_{n-l+1}(\mu)\right\} \sqrt{m_{l-1}(\mu) m_{l}(\mu)}- \\
& -\frac{b_{n-l+1}(\mu)\left[\delta_{j} m_{l-1}(\mu) m_{l}(\mu)+\delta_{j} m_{l}(\mu) m_{l-1}(\mu)\right]}{\sqrt{m_{l-1}(\mu) m_{l}(\mu)}} \\
& {\left.\left[\delta_{i} m_{l-1}(\mu) m_{l}(\mu)+\delta_{i} m_{l}(\mu) m_{l-1}(\mu)\right]\right] / 4 m_{l-1}(\mu) m_{l}(\mu)+} \\
& +\frac{\delta_{j} b_{n-l+1}(\mu)\left[\delta_{i} m_{l-1}(\mu) m_{l}(\mu)+\delta_{i} m_{l}(\mu) m_{l-1}(\mu)\right]}{2 \sqrt{m_{l-1}(\mu) m_{l}(\mu)}}+ \\
& +\sqrt{m_{l-1}(\mu) m_{l}(\mu)} \delta_{i} \delta_{j} b_{n-l+1}(\mu)
\end{aligned}
$$

para $l=2,3, \ldots, n, \mathrm{e}$

$\delta_{i} \delta_{j} k_{1}(\mu)=\delta_{i} \delta_{j} m_{1}(\mu) a_{n}(\mu)+\delta_{j} m_{1}(\mu) \delta_{i} a_{n}(\mu)+\delta_{i} \delta_{j} a_{n}(\mu) m_{1}(\mu)+\delta_{j} a_{n}(\mu) \delta_{i} m_{1}(\mu)+\delta_{i} \delta_{j} k_{2}(\mu)$. 
Com a mesma informação do algoritmo 4.1 as eqs obtidas a cima podem resumir-se no seguinte:

\section{ALGORITMO 4.2:}

Calcular $\left(a_{l}(\mu)\right)_{l=1}^{n}$, e $\left(b_{l}(\mu)\right)_{l=1}^{n-1}$ da matriz $J(\mu)$ usando o algoritmo de Lanczos 2.1

Determinar $\left(y_{l}(\mu)\right)_{l=1}^{n}$ resolvendo o sistema $J(\mu) y(\mu)=e_{1}$

Para $j=1,2, \ldots, n-1$

Determinar $\left(\delta_{j} y_{l}(\mu)\right)_{l=1}^{n}$ resolvendo o sistema $J(\mu) \delta_{j} y(\mu)=-\delta_{j} J(\mu) y(\mu)$

Determinar $\left(m_{l}(\mu)\right)_{l=1}^{n},\left(k_{l}(\mu)\right)_{l=1}^{n},\left(\delta_{j} m_{l}(\mu)\right)_{l=1}^{n},\left(\delta_{j} k_{l}(\mu)\right)_{l=1}^{n}$, usando o algoritmo 4.1

Para $i=1,2, \ldots, n-1$

Determinar $\left(\delta_{i} \delta_{j} y_{l}(\mu)\right)_{l=1}^{n}$ resolvendo o sistema $J(\mu) \delta_{i} \delta_{j} y(\mu)=-\left(\delta_{i} \delta_{j} J(\mu) y(\mu)+\right.$

$$
\begin{aligned}
\left.\delta_{i} J(\mu) \delta_{j} y(\mu)+\delta_{j} J(\mu) \delta_{i} y(\mu)\right) & {\left[\left(1+\sum_{l=1}^{n-1}\left(\frac{y_{l}(\mu)}{y_{n}(\mu)}\right)^{2}\right)^{2}\right.} \\
\text { Calcular } \delta_{i} \delta_{j} k_{1}(\mu)= & \left(-2 m_{T} \sum_{l=1}^{n-1}\left\{\frac{\left[\delta_{j} y_{l}(\mu) y_{n}^{3}(\mu)-3 y_{l}(\mu) y_{n}^{2}(\mu) \delta_{i} y_{n}(\mu)\right]}{y_{n}^{6}(\mu)}\right.\right. \\
& {\left[\delta_{j} y_{l}(\mu) y_{n}(\mu)-y_{l}(\mu) \delta_{j} y_{n}\right]\left[\delta_{i} \delta_{j} y_{l}(\mu) y_{n}(\mu)+\delta_{j} y_{l}(\mu) \delta_{i} y_{n}(\mu)-\right.} \\
& \left.\left.\left.\delta_{i} \delta_{j} y_{n}(\mu) y_{l}(\mu)-\delta_{j} y_{n}(\mu) \delta_{i} y_{l}(\mu)\right]\left(\frac{y_{l}(\mu)}{y_{n}^{3}(\mu)}\right)\right\}\right) \\
& \left(8 m_{T} \sum_{l=1}^{n-1} \frac{y_{l}(\mu)}{y_{n}^{3}(\mu)}\left[\delta_{j} y_{l}(\mu) y_{n}(\mu)-y_{l}(\mu) \delta_{j} y_{n}(\mu)\right]\right)\left(1+\sum_{l=1}^{n-1}\left(\frac{y_{l}(\mu)}{y_{n}(\mu)}\right)^{2}\right) \\
& \left.\left(\sum_{l=1}^{n-1} \frac{y_{l}(\mu)}{y_{n}^{3}(\mu)}\left[\delta_{i} y_{l}(\mu) y_{n}(\mu)-y_{l}(\mu) \delta_{i} y_{n}(\mu)\right]\right)\right] /\left[1+\sum_{l=1}^{n-1}\left(\frac{y_{l}(\mu)}{y_{n}(\mu)}\right)^{2}\right]^{4} \\
\delta_{i} \delta_{j} m_{l}(\mu)= & \delta_{i} \delta_{j} m_{n}(\mu)\left(\frac{y_{l}(\mu)}{y_{n}(\mu)}\right)^{2}+2 \delta_{j} m_{n}(\mu)\left(\frac{y_{l}(\mu)}{y_{n}^{3}(\mu)}\right)\left[\delta_{i} y_{l}(\mu) y_{n}(\mu)-\right.
\end{aligned}
$$




$$
\begin{aligned}
& \left.y_{l}(\mu) \delta_{i} y_{n}(\mu)\right] 2 \delta_{i} m_{n}(\mu)\left(\frac{y_{l}(\mu)}{y_{n}^{3}(\mu)}\right)\left[\delta_{j} y_{l}(\mu) y_{n}(\mu)-y_{l}(\mu) \delta_{j} y_{n}(\mu)\right] 2 m_{n}(\mu) \\
& \left(\frac{\left(\delta_{j} y_{l}(\mu) y_{n}^{3}(\mu)-3 y_{l}(\mu) y_{n}^{2}(\mu) \delta_{i} y_{n}(\mu)\right)}{y_{n}^{6}(\mu)}\right)\left[\delta_{j} y_{l}(\mu) y_{n}(\mu)-y_{l}(\mu) \delta_{j} y_{n}(\mu)\right] \\
& 2 m_{n}(\mu)\left(\frac{y_{l}(\mu)}{y_{n}^{3}(\mu)}\right)\left[\delta_{i} \delta_{j} y_{l}(\mu) y_{n}(\mu)+\delta_{j} y_{l}(\mu) \delta_{i} y_{n}(\mu)-\delta_{i} \delta_{j} y_{n}(\mu) y_{l}(\mu)-\right. \\
& \left.\delta_{j} y_{n}(\mu) \delta_{i} y_{l}(\mu)\right], l=1,2, \ldots, n-1 \\
\delta_{i} \delta_{j} k_{l}(\mu)= & {\left[2 \left\{\left[\delta_{i} \delta_{j} m_{l-1}(\mu) m_{l}(\mu)+\delta_{j} m_{l-1}(\mu) \delta_{i} m_{l}(\mu)+\delta_{i} \delta_{j} m_{l}(\mu) m_{l-1}(\mu)+\right.\right.\right.} \\
& \left.\delta_{j} m_{l}(\mu) \delta_{i} m_{l-1}(\mu)\right] b_{n-l+1}(\mu)+\left[\delta_{j} m_{l-1}(\mu) m_{l}(\mu) \delta_{j} m_{l}(\mu) m_{l-1}(\mu)\right] \\
& \left.\delta_{i} b_{n-l+1}(\mu)\right\} \sqrt{m_{l-1}(\mu) m_{l}(\mu)} \frac{b_{n-l+1}(\mu)\left[\delta_{j} m_{l-1}(\mu) m_{l}(\mu)+\delta_{j} m_{l}(\mu) m_{l-1}(\mu)\right.}{\sqrt{m_{l-1}(\mu) m_{l}(\mu)}} \\
& {\left.\left[\delta_{i} m_{l-1}(\mu) m_{l}(\mu)+\delta_{i} m_{l}(\mu) m_{l-1}(\mu)\right]\right] / 4 m_{l-1}(\mu) m_{l}(\mu) } \\
& \frac{\delta_{j} b_{n-l+1}(\mu)\left[\delta_{i} m_{l-1}(\mu) m_{l}(\mu)+\delta_{i} m_{l}(\mu) m_{l-1}(\mu)\right]}{2 \sqrt{m_{l-1}(\mu) m_{l}(\mu)}} \\
& \sqrt{m_{l-1}(\mu) m_{l}(\mu) \delta_{i} \delta_{j} b_{n-l+1}(\mu), l=2,3, \ldots, n} \\
\delta_{i} \delta_{j} k_{1}(\mu)= & \delta_{i} \delta_{j} m_{1}(\mu) a_{n}(\mu)+\delta_{j} m_{1}(\mu) \delta_{i} a_{n}(\mu)+\delta_{i} \delta_{j} a_{n}(\mu) m_{1}(\mu)+\delta_{j} a_{n}(\mu) \delta_{i} m_{1}(\mu)+ \\
&
\end{aligned}
$$

O algoritmo anterior é fundamental para obter a matriz Hessiana da função $F(\mu)$ necessária no Cap 5. Como a matriz Hessiana é simétrica, apenas é necessário calcular as derivadas de segunda ordem $\delta_{i} \delta_{j} M(\mu)$ e $\delta_{i} \delta_{j} K(\mu)$, para $i, j=1,2 \ldots, n-1$, o qual reduz consideravelmente o custo computacional . Alguns exemplos numéricos para ilustrar a eficiência dos algoritmos 4.1 e 4.2 são considerados na próxima Seção.

\subsection{Resultados Computacionais}

Os exemplos numéricos apresentados nesta Seção são usados para mostrar a eficiência e precisão dos métodos que calculam as derivadas de primeira e segunda ordem das matrizes massa e rigidez 
com relação ao espectro intercalado.

O primeiro exemplo mostra o gráfico das derivadas $\left(\delta_{j} m_{l}, \delta_{j} k_{l}\right)_{l=1}^{n-1}, j=1,2, \ldots, n-1$, das matrizes $M(\mu)$ e $K(\mu)$ correspondentes a dois sistemas massa-mola diferentes con $n$ graus de liberdade cada um. Posteriormente é ilustrado um exemplo que confirma a extatidão numérica do método. Neste caso é determinado um mínimo da função $F(\mu)$ usando o método de Newton, onde a convergência deve ser quadrática. Finalmente, é feita uma comparação do algoritmo 4.1 com uma aproximação por diferenças finitas. Esta comparação mostra claramente as vantagem do algoritmo 4.1 em precisão e eficiência .

\subsubsection{Gráficos das derivadas calculadas pelo algoritmo 4.1}

As derivadas das massas e constantes de rigidez de um sistema massa-mola calculadas pelo algoritmo 4.1 são calculadas para dois sistemas diferentes. Primeiro, um sistema com 10 graus de liberdade onde todas as massas são iguais a 1.0 e todas as constantes de rigidez iguais a 2.0 . $\mathrm{Na}$ figura 4.1 pode-se observar que as derivadas $\delta_{j} m_{l}$ e $\delta_{j} k_{l}$ com relação as últimas variáveis são próximas a zero. $\mathrm{O}$ sistema considerado tem um espectro onde os autovalores ficam muito próximos um do outro, como mostra a segunda coluna da Tabela 4.1. O espectro intercalado é listado na terceira coluna. Agora, quando é considerado um sistema com autovalores mais separados um do outro, temos o mesmo comportamento das derivadas, como é mostrado na Tabela 4.2. Os autovalores associados ao sistema aparecem na segunda e terceira colunas. $\mathrm{O}$ comportamento particular das derivadas com as últimas derivadas tendendo a zero podem ser exploradas para reduzir o tempo computacional, isto é, as últimas derivadas podem ser assumidas iguais a zero, e somente as primeiras devem ser calculadas.

\subsubsection{Derivada numérica exata das massas e constantes de rigidez}

A mesma idéia usada no Cap 3 para confirmar numericamente a precisão das derivadas calculadas pelo alogritmo $\delta$-Lanczos será considerada para o algoritmo 4.1. Usando o método de Newton é determinado um mínimo da função

$$
F(\mu)=\sum_{l=1}^{n}\left(m_{l}(\mu)-\bar{m}_{l}\right)^{2}+\sum_{l=1}^{n}\left(k_{l}(\mu)-\bar{k}_{l}\right)^{2},
$$

sujeito a $\bar{\lambda}_{i}<\mu_{i}<\bar{\lambda}_{i+1}, i=1,2, \ldots, n-1$, onde $\bar{\lambda}_{i}$ são os autovalores do sistema $(\bar{M}, \bar{K})$ com massas $\bar{m}_{i}$ e constantes de rigidez $\bar{k}_{i}$. O mínimo da função objetivo é o espectro intercalado $\bar{\mu}_{i}$ associado ao sistema $(\bar{M}, \bar{K})$. Quando é aplicado o método de Newton para determinar o ponto mínimo a convergência a $\overline{\mu_{i}}$ deve ser quadrática. 
Tabela 4.1:

\begin{tabular}{ccccc}
\hline \hline$i$ & $\lambda_{i}$ & $\mu_{i}$ & $m_{i}$ & $k_{i}$ \\
\hline \hline 1 & $4.467669 \mathrm{e}-02$ & 0.195773 & 1.000000 & 2.000000 \\
2 & 0.396124 & 0.763932 & 1.000000 & 2.000000 \\
3 & 1.067792 & 1.648858 & 1.000000 & 2.000000 \\
4 & 2.000000 & 2.763932 & 1.000000 & 2.000000 \\
5 & 3.109916 & 4.000000 & 1.000000 & 2.000000 \\
6 & 4.298920 & 5.236067 & 1.000000 & 2.000000 \\
7 & 5.461364 & 6.351141 & 1.000000 & 2.000000 \\
8 & 6.493959 & 7.236067 & 1.000000 & 2.000000 \\
9 & 7.304955 & 7.804226 & 1.000000 & 2.000000 \\
10 & 7.82229 & & 1.000000 & 2.000000 \\
\hline \hline
\end{tabular}

Tabela 4.2:

\begin{tabular}{ccccc}
\hline \hline$i$ & $\lambda_{i}$ & $\mu_{i}$ & $m_{i}$ & $k_{i}$ \\
\hline \hline 1 & 2.000000 & 4.100000 & 6.580885 & 620.718873 \\
2 & 25.200000 & 31.300000 & 0.943997 & 84.485946 \\
3 & 49.900000 & 59.100000 & 0.267563 & 17.315852 \\
4 & 68.600000 & 78.400000 & 0.338231 & 11.988623 \\
5 & 99.900000 & 110.200000 & 0.410301 & 19.743740 \\
6 & 123.300000 & 138.100000 & 0.520156 & 23.200994 \\
7 & 151.600000 & 159.200000 & 0.446633 & 32.037761 \\
8 & 174.100000 & 180.200000 & 0.309431 & 23.479891 \\
9 & 205.500000 & 222.70000 & 0.146469 & 11.599404 \\
10 & 233.30000 & & $3.633107 \mathrm{e}-02$ & 5.453294 \\
\hline \hline
\end{tabular}



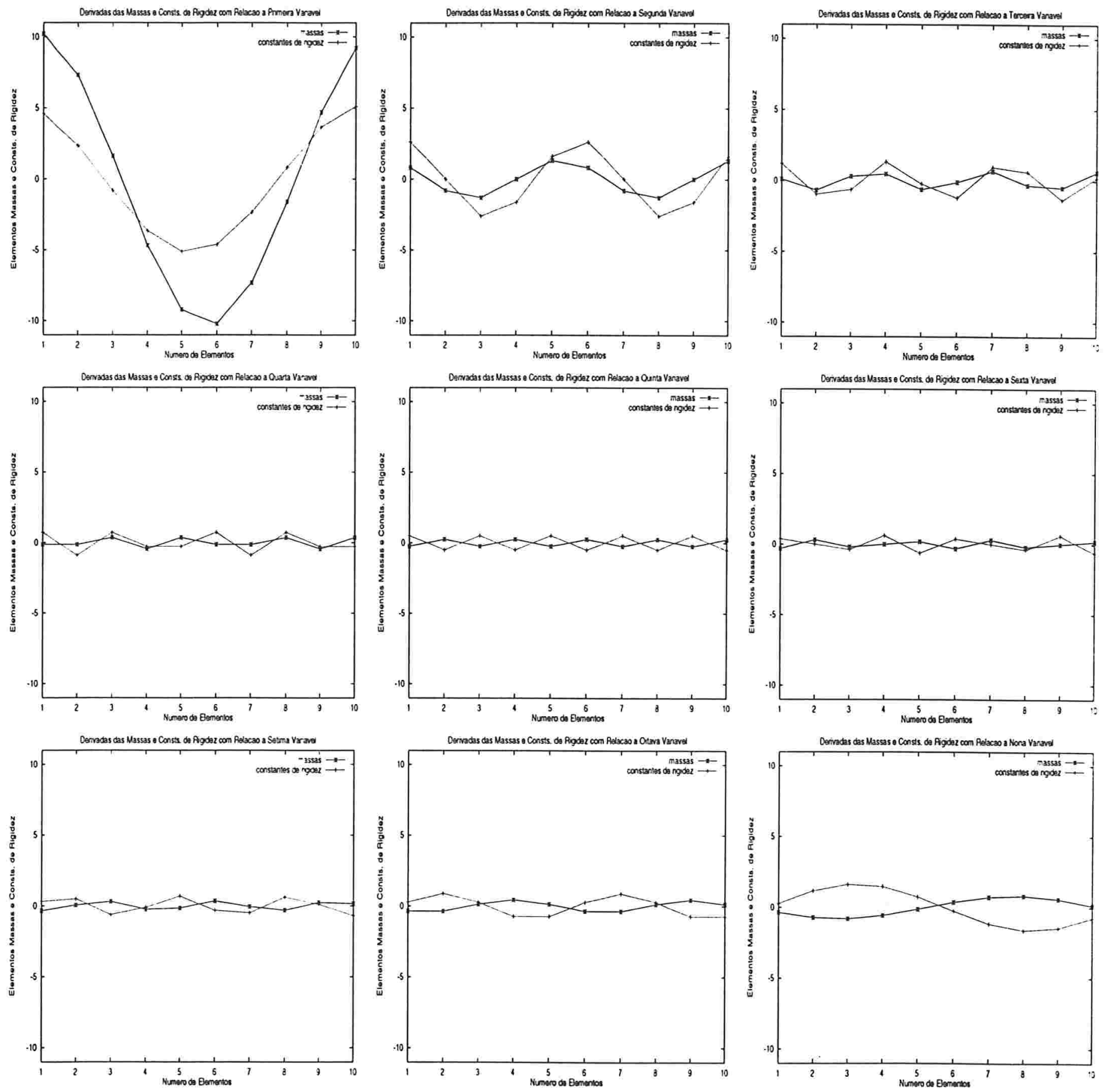

Figura 4.1: Gráficos das derivadas das massas e constantes de rigidez do sistema com 10 graus de liberdade, ilustrado na Tabela 4.1, com relação às nove variáveis. 

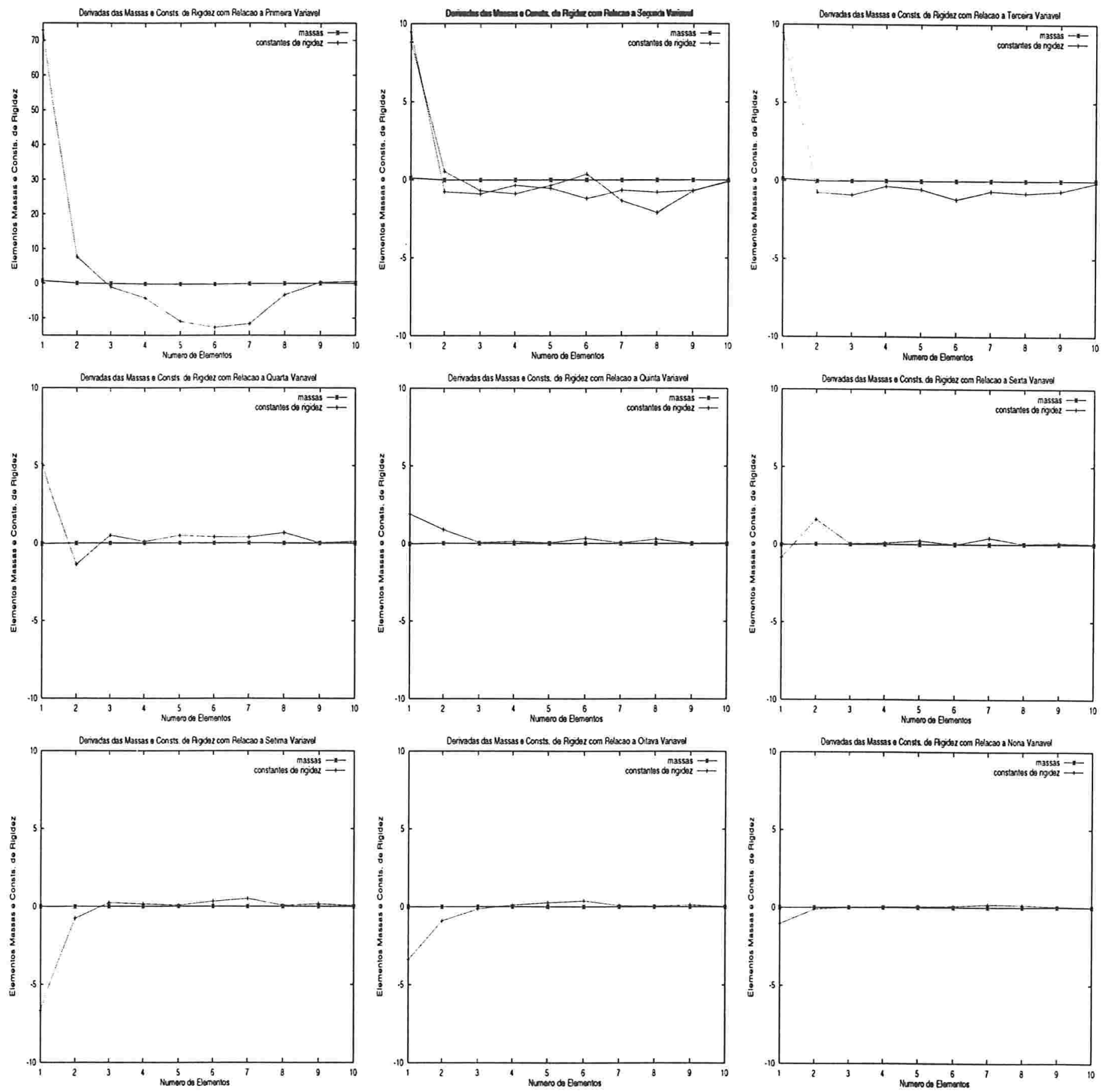

Figura 4.2: Gráficos das derivadas das massas e constantes de rigidez do sistema com 10 graus de liberdade, ilustrado na Tabela 4.2, com relação às nove variáveis. 


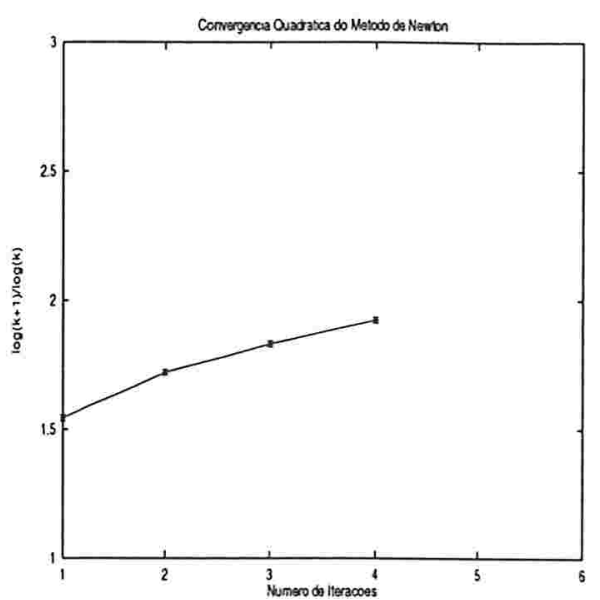

(a)

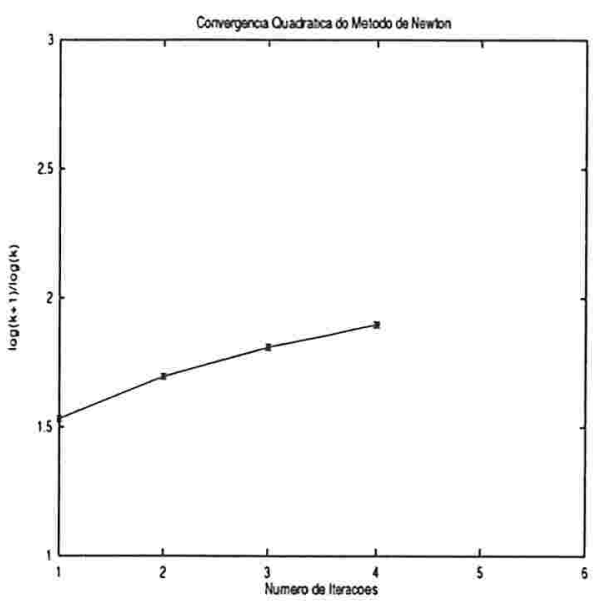

(b)

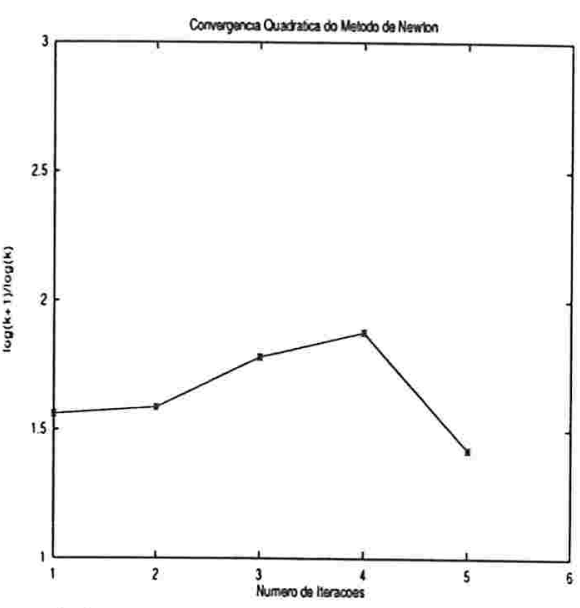

(c)

Figura 4.3: Gráficos dos $c_{j}=\log \left(e_{j+1}\right) / \log \left(e_{j}\right)$, onde $e_{j}$ denota o erro absoluto entre a solução exata e aproximada na j-ésima iteração do método de Newton para três valores inicias diferentes.

Tabela 4.3:

\begin{tabular}{cccccccc}
\hline \hline$i$ & $\bar{\lambda}_{i}$ & $\mu_{i}^{\text {exato }}$ & $\bar{m}_{i}$ & $\bar{k}_{i}$ & $1^{\circ} \mu_{i}^{v i}$ & $2^{\circ} \mu_{i}^{v i}$ & $3^{\circ} \mu_{i}^{v i}$ \\
\hline \hline 1 & 0.162028 & 0.763932 & 1.000000 & 2.000000 & 0.763932 & 0.863932 & 0.763932 \\
2 & 1.380557 & 2.763932 & 1.000000 & 2.000000 & 2.863932 & 2.763932 & 2.963932 \\
3 & 3.430740 & 5.236067 & 1.000000 & 2.000000 & 5.336067 & 5.336067 & 5.336067 \\
4 & 5.661660 & 7.236067 & 1.000000 & 2.000000 & 7.236067 & 7.236067 & 7.236067 \\
5 & 7.365014 & & 1.000000 & 2.000000 & & & \\
\hline \hline
\end{tabular}

As Figuras 4.3(a)-4.3(c) mostram os gráficos de $c_{j}=\log \left(e_{j+1}\right) / \log \left(e_{j}\right)$, onde $e_{j}$, como na Seção 3.4.4, denota o erro absoluto entre a solução exata e aproximada na $j$-ésima iteração para três valores iniciais diferentes. Neste caso é considerado um sistema com 5 graus de liberdades, onde as massas e constantes de rigidez são listadas na quarta e quinta coluna da Tabela 4.3, respectivamente. Para as três estimativas iniciais diferentes listadas nas três últimas colunas obtém-se valores de $c_{j}$ próximos a 2.0 . 


\subsubsection{Comparação do algoritmo 4.1 com diferenças finitas}

O esquema de diferenças centradas de segunda ordem é usado para comparação com o algoritmo 4.1, onde são calculadas as derivadas das matrizes massa e rigidez com relação a $\mu_{2}$. Um sistema com $m_{l}=1.0$ e $k_{l}=2.0, l=1,2, \ldots n$, para $n=10$ e $n=160$ é considerado. São definidos os erros $\hat{e}_{m}=\frac{\left\|m-m^{d f}\right\|_{\infty}}{\|m\|_{\infty}}, \hat{e}_{k}=\frac{\left\|k-k^{d f}\right\|_{\infty}}{\|k\|_{\infty}}$, onde $m, k$ são os vetores das massas e constantes de rigidez calculados pelo algoritmo 4.1 , e $m^{d f}, k^{d f}$ os calculados pelo esquema diferenças centradas.

As Tabelas 4.4 e 4.5 mostram os erros relativos para o sistema com 10 e 160 graus de liberdade, respectivamente. Claramente se observa a limitação da aproximação por diferenças finitas que depende da escolha de um valor ótimo de $\Delta \mu_{i}$. No caso do sistema com $n=10$ o passo ótimo é $\Delta \mu_{i}=1.0 e-07$, embora $\Delta \mu_{i}=1.0 e-09$ é o passo ótimo para um sistema maior com $n=160$. Além disso, evidentemente o custo computacional da aproximação por diferenças finitas não é competitiva com o algoritmo 4.1. mesmo assim o teste valida o algoritmo 4.1.

Tabela 4.4:

\begin{tabular}{ccc}
\hline \hline$\Delta \mu_{2}$ & $\delta_{2} \hat{e}_{m}$ & $\delta_{2} \hat{e}_{k}$ \\
\hline \hline $1.0 \mathrm{e}-03$ & $5.118003 \mathrm{e}-03$ & $5.464489 \mathrm{e}-03$ \\
$1.0 \mathrm{e}-04$ & $5.108939 \mathrm{e}-04$ & $5.453706 \mathrm{e}-04$ \\
$1.0 \mathrm{e}-05$ & $5.108047 \mathrm{e}-05$ & $5.456205 \mathrm{e}-05$ \\
$1.0 \mathrm{e}-06$ & $5.113293 \mathrm{e}-06$ & $5.450948 \mathrm{e}-06$ \\
$1.0 \mathrm{e}-07$ & $5.399376 \mathrm{e}-07$ & $5.261679 \mathrm{e}-07$ \\
$1.0 \mathrm{e}-08$ & $6.843436 \mathrm{e}-07$ & $7.248311 \mathrm{e}-07$ \\
$1.0 \mathrm{e}-09$ & $3.033677 \mathrm{e}-06$ & $9.348926 \mathrm{e}-06$ \\
$1.0 \mathrm{e}-10$ & $8.288555 \mathrm{e}-05$ & $8.700465 \mathrm{e}-05$ \\
$1.0 \mathrm{e}-11$ & $1.304199 \mathrm{e}-03$ & $1.397476 \mathrm{e}-03$ \\
\hline \hline
\end{tabular}


Tabela 4.5:

\begin{tabular}{ccc}
\hline \hline$\Delta \mu_{2}$ & $\delta_{2} \hat{e}_{m}$ & $\delta_{2} \hat{e}_{k}$ \\
\hline \hline $1.0 \mathrm{e}-06$ & $1.297757 \mathrm{e}-03$ & $1.298365 \mathrm{e}-03$ \\
$1.0 \mathrm{e}-07$ & $1.296742 \mathrm{e}-04$ & $1.297913 \mathrm{e}-04$ \\
$1.0 \mathrm{e}-08$ & $1.410730 \mathrm{e}-05$ & $1.413349 \mathrm{e}-05$ \\
$1.0 \mathrm{e}-09$ & $5.260449 \mathrm{e}-06$ & $5.261932 \mathrm{e}-06$ \\
$1.0 \mathrm{e}-10$ & $1.866978 \mathrm{e}-04$ & $1.867709 \mathrm{e}-04$ \\
$1.0 \mathrm{e}-11$ & $1.095633 \mathrm{e}-03$ & $1.099023 \mathrm{e}-03$ \\
\hline \hline
\end{tabular}




\section{Capítulo 5}

\section{Estratégia Multidimensional para o Isolamento de Frequências Naturais em Sistemas Discretos Massa-Mola}

O principal objetivo deste trabalho é investigar uma metodologia de solução eficiente e robusta do problema IFSMM. Se é considerado o sistema massa-mola inicial $(\bar{M}, \bar{K})$, onde um ou mais autovalores $\bar{\lambda}_{l}$ estão dentro de uma banda de ressonância $\beta(c ; r)$, então, usando as eqs $2.31,2.32$, e 2.33, deseja-se definir um novo espectro objetivo $\left(\lambda_{l}^{*}\right)_{1}^{n}$ livre de ressonância. Define-se a função $F: \Re^{2 n+1} \longrightarrow \Re$ por

$$
F(m, k, \alpha)=\sum_{l=1}^{n}\left(m_{l}-\bar{m}_{l}\right)^{2}+\sum_{l=1}^{n}\left(k_{l}-\bar{k}_{l}\right)^{2}+\alpha \sum_{l=1}^{n}\left(\lambda_{l}(m, k, \alpha)-\lambda_{l}^{*}\right)^{2},
$$

onde o parâmetro $\alpha$ é um multiplicador de Lagrange, e $m=\left(m_{1}, m_{2}, \ldots, m_{n}\right), k=\left(k_{1}, k_{2}, \ldots, k_{n}\right)$ são os vetores das massas e constantes de rigidez do sistema $(M, K)$, com autovalores $\left(\lambda_{l}(m, k, \alpha)\right)_{1}^{n}$. Uma primeira tentativa para determinar uma solução do problema IFSMM é aplicar a chamada força bruta, isto é,

$$
\begin{aligned}
\text { Minimizar } & F(m, k, \alpha) \\
\text { sujeito a : } & m_{l}, k_{l}>0, \quad l=1,2, \ldots, n
\end{aligned}
$$

Para resolver o problema 5.2 e- 5.3 , usando um processo de otimização convencional, é necessário calcular o vetor gradiente de $F$. De fato, o vetor gradiente é dado por 


$$
\begin{aligned}
\nabla F(m, k, \alpha)= & \left(\frac{\partial F(m, k, \alpha)}{\partial m_{1}}, \frac{\partial F(m, k, \alpha)}{\partial m_{2}}, \ldots, \frac{\partial F(m, k, \alpha)}{\partial m_{n}}, \frac{\partial F(m, k, \alpha)}{\partial k_{1}}, \frac{\partial F(m, k, \alpha)}{\partial k_{2}}, \ldots\right. \\
& \left.\ldots, \frac{\partial F(m, k, \alpha)}{\partial k_{n}}, \frac{\partial F(m, k, \alpha)}{\partial \alpha}\right),
\end{aligned}
$$

onde

$$
\begin{aligned}
\frac{\partial F(m, k, \alpha)}{\partial m_{j}}= & 2\left(m_{j}-\bar{m}_{j}\right)-2 \alpha \sum_{l=1}^{n} \lambda_{l}(m, k, \alpha)\left(\lambda_{l}(m, k, \alpha)-\lambda_{l}^{*}\right)\left(u j^{l}(m, k, \alpha)\right)^{2}, \\
\frac{\partial F(m, k, \alpha)}{\partial k_{j}}= & 2\left(k_{j}-\bar{k}_{j}\right)-2 \alpha \sum_{l=1}^{n} \lambda_{l}(m, k, \alpha)\left(\lambda_{l}(m, k, \alpha)-\lambda_{l}^{*}\right)\left(u_{j-1}^{l}(m, k, \alpha)-u_{j}^{l}(m, k, \alpha)\right)^{2}, \\
& u_{0}^{l}(m, k, \alpha)=0, j=1,2, \ldots, n, \\
\frac{\partial F(m, k, \alpha)}{\partial \alpha}= & \sum_{l=1}^{n} \lambda_{l}(m, k, \alpha)\left(\lambda_{l}(m, k, \alpha)-\lambda_{l}^{*}\right)^{2},
\end{aligned}
$$

com $u_{j}^{l}(m, k, \alpha)$ os autovetores associados aos autovalores $\lambda_{l}(m, k, \alpha)$. Então, em cada passo do processo de minimização, precisa-se calcular os autovalores e autovetores de $(M, K)$. Portanto, é evidente que o procedimento é computacionalmente caro, embora seja seguro. Este fato motiva o desenvolvimento de uma técnica mais sofisticada e eficiente do ponto de vista numérico.

No Cap 2 foi desenvolvida uma técnica inversa, onde um espectro intercalado $\mu$ é utilizado como control para minimizar o impacto de re-desenho. A busca unidimensional de $\mu$ foi a primeira estratégia usada, onde os resultados numéricos confirmam que a técnica inversa funciona. Mas, tudo indica que uma busca multidimensional do espectro intercalado deveria produzir melhores resultados. Neste caso, a função objetivo é redefinida por $F: \Re^{n-1} \longrightarrow \Re$ onde

$$
F(\mu)=\sum_{l=1}^{n}\left(m_{l}(\mu)-\bar{m}_{l}\right)^{2}+\sum_{l=1}^{n}\left(k_{l}(\mu)-\bar{k}_{l}\right)^{2} .
$$

O problema torna-se:

$$
\begin{array}{rc}
\text { Minimizar } & F(\mu) \\
\text { sujeito a : } & \lambda_{l}^{*}<\mu_{l}<\lambda_{l+1}^{*}
\end{array}
$$

A nova formulação do problema disminui o número de variáveis da função objetivo, e a reconstrução inversa garante que o sistema obtido tem os autovalores desejados. Neste Capítulo o problema 5.5, 5.6 é resolvido usando um método de minimização do tipo Lagrangiano aumentado com restrições de canalização desenvolvido por A. Friedlander, J.M. Martinez, e S.A. Santos [17], que será chamado método Box (Ver Apêndice A). 


\subsection{Estratégia multidimensional para matrizes de Jacobi}

Nesta Seção um estudo prévio da estratégia multidimensional para as matrizes de Jacobi é realizado. Aqui é formulado um problema para este tipo de matrizes, de maneira semelhante a 5.5 - 5.6, onde é resolvido usando o método de minimização Box. Os resultados numéricos obtidos mostram a eficiência e precisão da estrategia inversa, e servem como referência para a estratégia multidimensional aplicada em sistemas massa-mola. Na Seção seguinte são apresentados alguns resultados da estratégia aplicada ao problema 5.5 - 5.6, onde os autovalores não são isolados ainda.

Considerar a matriz de Jacobi $\bar{J}$ com elementos diagonais e co-diagonais $\left(\bar{a}_{l}\right)_{1}^{n}$ e $\left(\bar{b}_{l}\right)_{1}^{n-1}$, respectivamente. Os autovalores de $\bar{J}$ são denotados por $\left(\bar{\lambda}_{l}\right)_{1}^{n}$. Então, será resolvido o seguinte problema:

$$
\begin{array}{cc}
\text { Minimizar } & F(\mu) \\
\text { sujeito a : } & \bar{\lambda}_{l}<\mu_{l}<\bar{\lambda}_{l+1}, \quad l=1,2, \ldots, n-1
\end{array}
$$

onde a funçăo objetivo é dada por:

$$
F(\mu)=\sum_{l=1}^{n}\left(a_{l}(\mu)-\bar{a}_{l}\right)^{2}+\sum_{l=1}^{n-1}\left(b_{l}(\mu)-\bar{b}_{l}\right)^{2}
$$

Observe-se que neste caso não é considerado o isolamento de frequências, já que pretende-se comprovar a precisão e eficiência da estratégia.

Para resolver o problema 5.7 - 5.8 será usado o método de minimização Box. O método é do tipo região-de confiança que usa técnicas de Lagrangiano aumentado para minimizar uma função diferenciável de várias variáveis com restrições de canalização, como em 5.8. Em cada iteração o algoritmo, considera o problema de minimizar uma função quadrática (não necessariamente convexa) em uma caixa que consiste na interseção da caixa original $\bar{\lambda}_{l} \leq \mu_{l} \leq \bar{\lambda}_{l+1}$ com a regiãode confiança. $\mathrm{O}$ método resolve o subproblema em um número finito de passos, e a convergência do algoritmo principal é global. Uma descrição geral do método encontra-se no Apêndice $\mathrm{A}$. Na implementação, é necessário calcular, precisamente, o vetor gradiente e a matriz Hessiana da função objetivo.

$\mathrm{O}$ vetor gradiente de $F$ em 5.9 é dado por

$$
\nabla F(\mu)=\left(\frac{\partial F(\mu)}{\partial \mu_{1}}, \frac{\partial F(\mu)}{\partial \mu_{2}}, \ldots, \frac{\partial F(\mu)}{\partial \mu_{n-1}}\right)
$$


Tabela 5.1:

\begin{tabular}{ccccc}
\hline \hline$l$ & $\bar{\lambda}_{l}$ & $\bar{\mu}_{l}$ & $\bar{a}_{l}$ & $\bar{b}_{l}$ \\
\hline 1 & 2.000000 & 4.100000 & 107.159567 & 33.896662 \\
2 & 25.200000 & 31.300000 & 107.841199 & 34.454446 \\
3 & 49.900000 & 59.100000 & 109.523548 & 39.851889 \\
4 & 68.600000 & 78.400000 & 93.818409 & 52.999396 \\
5 & 99.900000 & 110.200000 & 104.666395 & 50.221355 \\
6 & 123.300000 & 138.100000 & 106.196499 & 66.469090 \\
7 & 151.600000 & 159.200000 & 124.302522 & 63.159464 \\
8 & 174.100000 & 180.200000 & 113.367018 & 54.485367 \\
9 & 205.500000 & 222.70000 & 116.424839 & 74.756003 \\
10 & 233.30000 & & 150.100000 & \\
\hline \hline
\end{tabular}

onde

$$
\frac{\partial F(\mu)}{\partial \mu_{j}}=2\left(\sum_{l=1}^{n}\left(a_{l}(\mu)-\bar{a}_{l}\right) \frac{\partial a_{l}(\mu)}{\partial \mu_{j}}+\sum_{l=1}^{n-1}\left(b_{l}(\mu)-\bar{b}_{l}\right) \frac{\partial b_{l}(\mu)}{\partial \mu_{j}}\right),
$$

com $j=1,2, \ldots, n-1$. A matriz Hessiana de ordem $(n-1)$ é denotada por $\mathcal{H}(F(\mu))=$ $\left[\frac{\partial^{2} F(\mu)}{\partial \mu_{i} \partial \mu_{j}}\right]_{i, j}$, para $i, j=1,2, \ldots, n-1$, cujos elementos são

$\frac{\partial^{2} F(\mu)}{\partial \mu_{i} \partial \mu_{j}}=2\left[\sum_{l=1}^{n}\left(\frac{\partial a_{l}(\mu)}{\partial \mu_{i}} \cdot \frac{\partial a_{l}(\mu)}{\partial \mu_{j}}+\left(a_{l}(\mu)-\bar{a}_{l}\right) \frac{\partial^{2} a_{l}(\mu)}{\partial \mu_{i} \partial \mu_{j}}\right)+\sum_{l=1}^{n-1}\left(\frac{\partial b_{l}(\mu)}{\partial \mu_{i}} \cdot \frac{\partial b_{l}(\mu)}{\partial \mu_{j}}+\left(b_{l}(\mu)-\bar{b}_{l}\right) \frac{\partial^{2} b_{l}(\mu)}{\partial \mu_{i} \partial \mu_{j}}\right)\right]$.

O vetor $\nabla F(\mu)$ e a matriz $\mathcal{H}(F(\mu))$ são calculados exatamente pelo algoritmo $\delta^{2}$-Lanczos discutido no Cap 3.

Agora são apresentados dois exemplos para mostrar a eficiência e precisão da estratégia multidimensional. Primeiro considera-se uma matriz de Jacobi $\bar{J}$ de ordem $n=10$, reconstruída a partir dos autovalores $\left(\bar{\lambda}_{l}\right)_{1}^{n}$ e do espectro intercalado $\left(\bar{\mu}_{l}\right)_{1}^{n-1}$, listados nas segunda e terceira colunas da Tabela 5.1. Os elementos diagonais e co-diagonais são listados nas últimas duas colunas da Tabela 5.1 . 
Tabela 5.2:

\begin{tabular}{c||cccc}
\hline \hline Teste & 1 & 2 & 3 & 4 \\
\hline Semente & -32 & -534 & -874 & -134 \\
\hline & 20.664984 & 3.939989 & 10.435355 & 17.266867 \\
& 25.587827 & 25.661028 & 31.269730 & 47.004189 \\
& 65.193392 & 55.182609 & 58.449697 & 56.322310 \\
& 77.377424 & 80.987565 & 99.797519 & 71.418712 \\
Valor Inicial & 105.037475 & 105.801215 & 118.048343 & 107.081715 \\
$\mu_{l}^{v i}$ & 138.353516 & 133.326691 & 147.340775 & 138.056062 \\
& 157.834363 & 163.528796 & 163.794645 & 157.196778 \\
& 185.502545 & 178.055610 & 205.392960 & 197.315508 \\
& 221.315283 & 208.461471 & 231.529263 & 222.587991 \\
\hline$F\left(\mu^{*}\right)$ & $\mathbf{5 . 0 2 6 3 4 9 e - 2 3}$ & $\mathbf{5 . 0 5 2 8 3 9 e - 2 3}$ & $\mathbf{5 . 0 3 8 4 7 1 \mathrm { e } - 2 3}$ & $\mathbf{5 . 0 4 0 6 1 1 \mathrm { e } - 2 3}$ \\
\hline$\left\|\nabla F\left(\mu^{*}\right)\right\|$ & $1.096885 \mathrm{e}-12$ & $7.598532 \mathrm{e}-13$ & $9.134648 \mathrm{e}-13$ & $6.694171 \mathrm{e}-13$ \\
\hline$N^{o}$ Iteraçöes & 18 & 17 & 30 & 16 \\
do Box & & & & \\
\hline \hline
\end{tabular}

O exemplo consiste em usar o método de minimização Box a partir de um valor inicial gerado aleatoriamente. Se $\mu^{*}$ é a solução obtida depois de aplicar o método Box, então o resultado ideal é que a função objetivo e o vetor gradiente anulam-se em $\mu^{*}$, isto é, $F\left(\mu^{*}\right)=\left\|\nabla F\left(\mu^{*}\right)\right\|=0$.

Para aplicar o algoritmo Box é necessário um valor inicial $\left(\mu_{l}^{v i}\right)_{1}^{n-1}$ tal que $\bar{\lambda}_{l}<\mu_{l}^{v i}<\bar{\lambda}_{l+1}$. Esses valores serão gerados de maneira aleatória. A sequência de números aleatórios $\left(r_{l}\right)_{1}^{n-1}$, com $0<r_{l}<1$, gerados a partir de um número inteiro negativo (semente), produzem os $\left(\mu_{l}\right)_{1}^{n-1}$. Uma vez gerados os $r_{l}$ (usando a função ran1(idum) (Ver Pág 271 [46])) os $\mu_{l}^{v i}$ são definidos tal que a propriedade de intercalamento seja satisfeita, isto é,

$$
\mu_{l}^{v i}=r_{l}\left(\bar{\lambda}_{l+1}-\bar{\lambda}_{l}\right)+\bar{\lambda}_{l}
$$

para $l=1,2, \ldots, n-1$.

Para a matriz especificada na Tabela 5.1 são considerados quatro valores iniciais diferentes gerados aleatoriamente, como se mostra na Tabela 5.2. Claramente, os resultados são excelentes e confirmam numericamente a eficiência e precisão da estratégia multidimensional para matrizes de Jacobi. Além disso, o número de iterações depende da precisão desejada.

Resultados semelhantes são obtidos quando é considerada uma matriz de ordem maior. A 
Tabela 5.3:

\begin{tabular}{ccccc}
\hline \hline$l$ & $\bar{\lambda}_{l}$ & $\bar{\mu}_{l}$ & $\bar{a}_{l}$ & $\bar{b}_{l}$ \\
\hline \hline 1 & 2.000000 & 4.100000 & 282.029006 & 34.587074 \\
2 & 25.200000 & 31.300000 & 306.464908 & 36.426390 \\
3 & 49.900000 & 59.100000 & 326.067187 & 69.587190 \\
4 & 68.600000 & 78.400000 & 390.287454 & 63.301072 \\
5 & 99.900000 & 110.200000 & 367.011759 & 96.169085 \\
6 & 123.300000 & 138.100000 & 379.924427 & 109.084714 \\
7 & 151.600000 & 159.200000 & 345.579715 & 105.377488 \\
8 & 174.100000 & 180.200000 & 331.163895 & 114.039434 \\
9 & 205.500000 & 222.70000 & 374.566907 & 108.208757 \\
10 & 233.30000 & 239.500000 & 347.969239 & 131.367745 \\
$\vdots$ & $\vdots$ & $\vdots$ & $\vdots$ & $\vdots$ \\
21 & 471.400000 & 488.800000 & 366.404336 & 172.092847 \\
22 & 499.900000 & 518.600000 & 372.180394 & 186.380674 \\
23 & 526.300000 & 538.700000 & 344.624625 & 177.687484 \\
24 & 546.600000 & 557.900000 & 360.263570 & 172.027022 \\
25 & 566.700000 & 577.700000 & 334.364440 & 189.394845 \\
26 & 584.500000 & 599.100000 & 363.853506 & 177.399042 \\
27 & 617.700000 & 633.400000 & 352.799364 & 179.046412 \\
28 & 649.100000 & 673.900000 & 357.379883 & 188.856109 \\
29 & 691.600000 & 702.900000 & 380.178400 & 234.019635 \\
30 & 715.800000 & & 373.600000 & \\
\hline \hline & & & &
\end{tabular}


Tabela 5.4:

\begin{tabular}{|c|c|c|c|c|}
\hline Teste & 1 & 2 & 3 & 4 \\
\hline Semente & -97 & -34 & -645 & -743 \\
\hline \multirow{10}{*}{$\begin{array}{l}\text { Valor Inicial } \\
\qquad\left(\mu_{l}^{v i}\right)_{1}^{10}\end{array}$} & 22.900777 & 3.977444 & 20.067230 & 15.854319 \\
\hline & 38.014544 & 36.660210 & 35.456568 & 29.231767 \\
\hline & 62.950479 & 56.977872 & 54.093007 & 66.737914 \\
\hline & 77.941897 & 93.472472 & 95.541667 & 74.712660 \\
\hline & 100.660531 & 122.316893 & 121.263270 & 104.861582 \\
\hline & 130.820885 & 130.584935 & 129.873926 & 146.811493 \\
\hline & 163.480189 & 160.886110 & 167.101698 & 157.017602 \\
\hline & 182.286443 & 174.736601 & 201.442076 & 189.040448 \\
\hline & 213.389595 & 205.845821 & 210.442076 & 218.426908 \\
\hline & 239.372340 & 241.596967 & 245.378757 & 237.519077 \\
\hline \multirow{11}{*}{$\begin{array}{l}\text { Valor Inicial } \\
\qquad\left(\mu_{l}^{v i}\right)_{21}^{30}\end{array}$} & $\vdots$ & $\vdots$ & $\vdots$ & $\vdots$ \\
\hline & 452.266785 & 457.869049 & 456.639647 & 469.724618 \\
\hline & 493.558632 & 478.351692 & 472.223221 & 478.575039 \\
\hline & 515.571818 & 510.678833 & 519.780418 & 511.030757 \\
\hline & 545.389932 & 526.970209 & 531.983793 & 530.857665 \\
\hline & 549.274538 & 558.580716 & 556.222213 & 547.583272 \\
\hline & 575.950524 & 571.462921 & 566.808746 & 583.384678 \\
\hline & 607.045893 & 592.781370 & 595.954566 & 610.149579 \\
\hline & 641.933566 & 646.572857 & 620.536234 & 623.503309 \\
\hline & 689.649098 & 681.897332 & 672.608771 & 653.639470 \\
\hline & 707.493907 & 696.379149 & 701.167576 & 704.789958 \\
\hline$F\left(\mu^{*}\right)$ & $2.373702 \mathrm{e}-22$ & $2.397967 \mathrm{e}-22$ & $2.386921 \mathrm{e}-22$ & $2.368195 \mathrm{e}-22$ \\
\hline$\left\|\nabla F\left(\mu^{*}\right)\right\|$ & $7.967346 \mathrm{e}-12$ & $1.014289 \mathrm{e}-11$ & $8.220153 \mathrm{e}-12$ & $9.466727 \mathrm{e}-12$ \\
\hline $\begin{array}{c}N^{\circ} \text { Iteraçoes } \\
\text { do Box }\end{array}$ & 18 & 30 & 22 & 22 \\
\hline
\end{tabular}


Tabela 5.3 ilustra os primeiros e últimos dez autovalores e elementos de uma matriz de ordem $n=30$. Para os valores iniciais listados na Tabela 5.4 os resultados novamente são excelentes.

Observe-se que as restrições de canalização 5.8 não permitem um bom desempenho do método Box quando o espaçamento dos autovalores é pequeno. Neste caso, é necessário usar métodos de otimização mais eficientes e robustos.

\subsection{Estratégia multidimensional para sistemas massa-mola}

Os resultados obtidos na Seção anterior sugerem que a estratégia multidimiensional aplicada a sistemas massa-mola também deve ser eficiente e exata.

\subsubsection{Massa total constante}

Primeiro é considerado o problema 5.5 - 5.6, isto é, deve-se achar um ponto mínimo da função

$$
F(\mu)=\sum_{l=1}^{n}\left(m_{l}(\mu)-\bar{m}_{l}\right)^{2}+\sum_{l=1}^{n}\left(k_{l}(\mu)-\bar{k}_{l}\right)^{2}
$$

onde $\mu=\left(\mu_{1}, \mu_{2}, \ldots, \mu_{n-1}\right)$ é sujeito à restrição de canalização

$$
\bar{\lambda}_{l}<\mu_{l}<\bar{\lambda}_{l+1}, \quad l=1,2, \ldots, n-1,
$$

onde os $\bar{\lambda}_{l}$ correspondem aos autovalores do sistema inicial $(\bar{M}, \bar{K})$. Numa primeira etapa os autovalores $\bar{\lambda}_{l}$ suponham-se livre de ressonância, isto é, dada uma banda de ressonância $\beta(c ; r)$ , não existe nenhum autovalor $\bar{\lambda}_{l}$ contido em $\beta(c ; r)$. Observa-se que, de acordo à formulação do problema, na reconstrução do sistema, a massa total $m_{T}$ é considerada constante.

Aqui, como na Seção anterior, será usado o algoritmo Box para determinar um ponto estacionário de $F$ com a restrição de canalização de acima. O vetor gradiente e a matriz Hessiana são obtidos do algoritmo 4.2, o qual calcula as derivadas de primeira e segunda ordem das massas e constantes de rigidez de um sistema massa-mola.

No primeiro exemplo é considerado um sistema com 10 graus de liberdade, cujas massas e constantes de rigidez são listadas na Tabela 5.5. Os autovalores e espectro intercalado do sistema correspondem aos mesmos utilizados no primeiro exemplo da Seção 5.1.

O valor inicial $\mu_{l}^{v i}$ é gerado por 5.12 , isto é, 
Tabela 5.5:

\begin{tabular}{ccccc}
\hline \hline$l$ & $\bar{\lambda}_{l}$ & $\bar{\mu}_{l}$ & $\bar{m}_{l}$ & $\bar{k}_{l}$ \\
\hline \hline 1 & 2.000000 & 4.100000 & 6.580885 & 620.718873 \\
2 & 25.200000 & 31.300000 & 0.943997 & 84.485946 \\
3 & 49.900000 & 59.100000 & 0.267563 & 17.315852 \\
4 & 68.600000 & 78.400000 & 0.338231 & 11.988623 \\
5 & 99.900000 & 110.200000 & 0.410301 & 19.743740 \\
6 & 123.300000 & 138.100000 & 0.520156 & 23.200994 \\
7 & 151.600000 & 159.200000 & 0.446633 & 32.037761 \\
8 & 174.100000 & 180.200000 & 0.309431 & 23.479891 \\
9 & 205.500000 & 222.70000 & 0.146469 & 11.599404 \\
10 & 233.30000 & & 0.036331 & 5.453294 \\
\hline \hline
\end{tabular}

$$
\mu_{l}^{v i}=r_{l}\left(\bar{\lambda}_{l+1}-\bar{\lambda}_{l}\right)+\bar{\lambda}_{l}, l=1,2, \ldots, n-1,
$$

garantindo a propriedade de intercalamento dos autovalores. Note-se que, para os exemplos ilustrados nesta Seção, são gerados aleatoriamente 10 sementes a partir da semente igual a -431. Se $\mu^{*}$ é o ponto mínimo de $F$, e os autovalores não foram isolados (não perturbados), o resultado ideal é $F\left(\mu^{*}\right)=\left\|\nabla F\left(\mu^{*}\right)\right\|=0$.

Para os valores iniciais listados na Tabela 5.6, o método só determina mínimos locais exceto em dois casos, e em outros não converge. Este fato, a princípio, foi atribuído a que o valor inicial fica muito próximo dos autovalores $\bar{\lambda}_{l}$, o que não permitiria encontrar uma direção de descida à $\mu^{*}$. Uma forma de evitar este problema é redefinir o valor inicial aleatório, de maneira de manter um mínimo de afastamento dos $\bar{\lambda}_{l}$. Se cada elemento $\mu_{l}^{v i}$ fica a uma distância $\varepsilon_{l}$ do correspondente $\bar{\lambda}_{l}$, então o valor inicial é gerado por

$$
\mu_{l}^{v i}=r_{l}\left(\bar{\lambda}_{l+1}-\bar{\lambda}_{l}-2 \varepsilon_{l}\right)+\bar{\lambda}_{l}+\varepsilon_{l}, l=1,2, \ldots, n-1,
$$

onde $\varepsilon_{l}=\frac{\bar{\lambda}_{l+1}-\bar{\lambda}_{l}}{d}$, com $d>2$..

A Tabela 5.7 mostra os novos resultados quando os chutes iniciais foram gerados por 5.14. Neste caso, os resultados melhoram, mas o ponto mínimo é atingido só em $40 \%$ dos casos.

Experimentos numéricos indicam que existem vários mínimos locais em torno de $\mu^{*}$, e provavelmente os mínimos locais ficam presos em pontos estacionários, nào permitindo encontrar uma 
Tabela 5.6:

\begin{tabular}{|c|c|c|c|c|c|}
\hline Teste & 1 & 2 & 3 & 4 & 5 \\
\hline Semente & -504 & -633 & -455 & -181 & -186 \\
\hline \multirow{9}{*}{$\begin{array}{c}\text { Valor Inicial } \\
\mu_{l}^{v i}\end{array}$} & 20.662851 & 3.719424 & 15.680315 & 6.927088 & 13.657698 \\
\hline & 40.763862 & 47.525689 & 27.557579 & 42.775738 & 44.027523 \\
\hline & 53.476818 & 59.129437 & 68.200008 & 66.969006 & 54.718536 \\
\hline & 94.417956 & 80.887076 & 85.429832 & 98.755699 & 84.506436 \\
\hline & 109.250164 & 111.880632 & 113.464484 & 100.280406 & 101.066478 \\
\hline & 132.075856 & 137.073669 & 125.585779 & 127.805034 & 144.289094 \\
\hline & 172.375311 & 159.735661 & 156.759909 & 158.531292 & 154.005053 \\
\hline & 188.468475 & 188.773248 & 196.560488 & 191.072328 & 205.190209 \\
\hline & 208.433486 & 207.687364 & 215.701386 & 210.998838 & 227.787323 \\
\hline$F\left(\mu^{*}\right)$ & $2.669187 \mathrm{e}-02$ & $6.637624 \mathrm{e}-02$ & 0.168722 & 2520.29 & 16554.89 \\
\hline$\left\|\nabla F\left(\mu^{*}\right)\right\|$ & $7.243332 \mathrm{e}-11$ & $4.281554 \mathrm{e}-12$ & $9.323253 \mathrm{e}-11$ & 51.623383 & $>1.0 \mathrm{e}+6$ \\
\hline $\begin{array}{c}N^{o} \text { Iterações } \\
\text { do Box }\end{array}$ & 91 & 39 & 143 & 78 & 37 \\
\hline Teste & 6 & 7 & 8 & 9 & 10 \\
\hline Semente & -458 & -634 & -901 & -727 & -565 \\
\hline \multirow{9}{*}{$\begin{array}{c}\text { Valor Inicial } \\
\mu_{l}^{v i}\end{array}$} & 4.049776 & 15.261962 & 2.245973 & 4.359547 & 8.381661 \\
\hline & 26.569971 & 44.619018 & 27.361948 & 31.337482 & 26.164114 \\
\hline & 63.441169 & 66.277270 & 67.995560 & 53.848205 & 56.901850 \\
\hline & 85.229397 & 94.884245 & 80.162132 & 75.815049 & 86.348819 \\
\hline & 107.405640 & 121.116196 & 111.313442 & 115.176130 & 117.409582 \\
\hline & 132.241843 & 143.695296 & 145.051031 & 133.435692 & 139.285143 \\
\hline & 157.991212 & 155.987345 & 162.605854 & 173.504946 & 167.294370 \\
\hline & 191.417028 & 188.809734 & 201.342231 & 191.507637 & 182.623442 \\
\hline & 226.392924 & 228.089196 & 211.753869 & 220.897028 & 207.041618 \\
\hline$F\left(\mu^{*}\right)$ & $1.521928 \mathrm{e}-24$ & 2535.73 & 0.168722 & $1.006438 \mathrm{e}-01$ & $5.814132 \mathrm{e}-26$ \\
\hline$\left\|\nabla F\left(\mu^{*}\right)\right\|$ & $1.834033 \mathrm{e}-10$ & 414.36 & $2.869545 \mathrm{e}-10$ & $9.447362 \mathrm{e}-10$ & $1.771964 \mathrm{e}-11$ \\
\hline $\begin{array}{c}N^{\circ} \text { Iterações } \\
\text { do Box }\end{array}$ & 95 & 74 & 50 & 61 & 145 \\
\hline
\end{tabular}


Tabela 5.7:

\begin{tabular}{|c|c|c|c|c|c|}
\hline Teste & 1 & 2 & 3 & 4 & 5 \\
\hline Semente & -504 & -633 & -455 & -181 & -186 \\
\hline \multirow{3}{*}{$d$} & 50.0 & 50.0 & 50.0 & 50.0 & 50.0 \\
\hline & 5.0 & 5.0 & & 5.0 & 5.0 \\
\hline & 2.5 & 2.5 & & 2.5 & 2.5 \\
\hline \multirow{3}{*}{$F\left(\mu^{*}\right)$} & 25080.55 & $6.637624 \mathrm{e}-02$ & $7.033769 \mathrm{e}-26$ & 2534.73 & 25080.5 .5 \\
\hline & 25080.55 & $2.669187 \mathrm{e}-02$ & & 0.168722 & 113.30 \\
\hline & $7.829929 \mathrm{e}-02$ & $6.637624 \mathrm{e}-02$ & & 0.168722 & 2736.11 \\
\hline \multirow{3}{*}{$\left\|\nabla F\left(\mu^{*}\right)\right\|$} & 43001.25 & $5.948187 \mathrm{e}-07$ & $1.388844 \mathrm{e}-11$ & $>1.0 \mathrm{e}+6$ & 26396.33 \\
\hline & 15474.44 & $2.816938 \mathrm{e}-10$ & 8.676728 & $7.570119 \mathrm{e}-11$ & 38382.94 \\
\hline & 5.864495 & $3.167124 \mathrm{e}-09$ & $5.333652 \mathrm{e}-11$ & $5.910829 \mathrm{e}-09$ & 65.02 \\
\hline \multirow{3}{*}{$\begin{array}{c}N^{\circ} \text { Iterações } \\
\text { do Box }\end{array}$} & 24 & 210 & 63 & 214 & 26 \\
\hline & 24 & 40 & & 86 & 66 \\
\hline & 53 & 52 & & 114 & 31 \\
\hline Teste & 6 & 7 & 8 & 9 & 10 \\
\hline Semente & -458 & -634 & -901 & -727 & -565 \\
\hline \multirow{3}{*}{$d$} & 50.0 & 50.0 & 50.0 & 50.0 & 50.0 \\
\hline & 5.0 & 5.0 & 5.0 & 5.0 & 5.0 \\
\hline & & 2.2 & 2.5 & & \\
\hline \multirow{3}{*}{$F\left(\mu^{*}\right)$} & $8.587594 \mathrm{e}-26$ & 2533.68 & $1.006438 \mathrm{e}-01$ & $1.006438 \mathrm{e}-01$ & $1.006438 \mathrm{e}-01$ \\
\hline & $7.864088 \mathrm{e}-26$ & 2238.42 & $1.006438 \mathrm{e}-01$ & $8.020049 \mathrm{e}-25$ & $6.753230 \mathrm{e}-26$ \\
\hline & & 0.168722 & $6.414607 \mathrm{e}-25$ & & \\
\hline \multirow{3}{*}{$\left\|\nabla F\left(\mu^{*}\right)\right\|$} & $1.009939 \mathrm{e}-11$ & 4134.78 & $9.573891 \mathrm{e}-10$ & $2.787080 \mathrm{e}-10$ & $3.011135 \mathrm{e}-07$ \\
\hline & $1.563983 \mathrm{e}-11$ & 8630.76 & $1.663860 \mathrm{e}-05$ & $1.275009 \mathrm{e}-10$ & $1.557475 \mathrm{e}-11$ \\
\hline & & $7.912420 \mathrm{e}-10$ & $1.152423 \mathrm{e}-10$ & & \\
\hline \multirow{3}{*}{$\begin{array}{c}N^{\circ} \text { Iterações } \\
\text { do Box }\end{array}$} & 88 & 36 & 46 & 52 & 65 \\
\hline & 158 & 189 & 41 & 109 & 474 \\
\hline & & 42 & 61 & & \\
\hline
\end{tabular}


direção de descida ao mínimo. Em todos os estudos anteriores a masa total $m_{T}$ foi considerada constante. Este fato sugere uma reformulação do problema, onde $m_{T}$ é considerada uma variável independente, permitindo relaxar o processo de minimização na busca do $\mu^{*}$. 


\subsubsection{Massa total como variável independente}

Se a massa total $m_{T}$ é considerada uma variável independente, então o problema variacional 5.7 - 5.8 torna-se:

$$
\begin{array}{cl}
\text { Minimizar } & F(\mu) \\
\text { sujeito a : } & \bar{\lambda}_{l}<\mu_{l}<\bar{\lambda}_{l+1}, l=1,2, \ldots, n-1 \\
& a<m_{T}<b, \quad b>a>0,
\end{array}
$$

onde a função objetivo é a mesma do 5.4 , só que agora $\mu \in \Re^{n}$, com $\mu=\left(\mu_{1}, \mu_{2}, \ldots, \mu_{n-1}, m_{T}\right)$. Se $\mu_{n} \equiv m_{T}$, o vetor gradiente será da forma

$$
\nabla F(\mu)=\left(\frac{\partial F(\mu)}{\partial \mu_{1}}, \frac{\partial F(\mu)}{\partial \mu_{2}}, \ldots, \frac{\partial F(\mu)}{\partial \mu_{n-1}}, \frac{\partial F(\mu)}{\partial \mu_{n}}\right)
$$

onde

$$
\frac{\partial F(\mu)}{\partial \mu_{j}}=2\left(\sum_{l=1}^{n}\left(m_{l}(\mu)-\bar{m}_{l}\right) \frac{\partial m_{l}(\mu)}{\partial \mu_{j}}+\sum_{l=1}^{n}\left(k_{l}(\mu)-\bar{k}_{l}\right) \frac{\partial k_{l}(\mu)}{\partial \mu_{j}}\right)
$$

$\operatorname{com} j=1,2, \ldots, n$. A matriz Hessiana $\mathcal{H}(F(\mu))=\left[\frac{\partial^{2} F(\mu)}{\partial \mu_{i} \partial \mu_{j}}\right]_{i, j}$ tem $n \times n$ elementos

$$
\begin{aligned}
\frac{\partial^{2} F(\mu)}{\partial \mu_{i} \partial \mu_{j}}= & 2\left[\sum_{l=1}^{n}\left(\frac{\partial m_{l}(\mu)}{\partial \mu_{i}} \cdot \frac{\partial m_{l}(\mu)}{\partial \mu_{j}}+\left(m_{l}(\mu)-\bar{m}_{l}\right) \frac{\partial^{2} m_{l}(\mu)}{\partial \mu_{i} \partial \mu_{j}}\right)\right. \\
& \left.+\sum_{l=1}^{n}\left(\frac{\partial k_{l}(\mu)}{\partial \mu_{i}} \cdot \frac{\partial k_{l}(\mu)}{\partial \mu_{j}}+\left(k_{l}(\mu)-\bar{k}_{l}\right) \frac{\partial^{2} k_{l}(\mu)}{\partial \mu_{i} \partial \mu_{j}}\right)\right],
\end{aligned}
$$

para $i, j=1,2, \ldots, n$. Por tanto, para obter $\nabla F(\mu)$ e $\mathcal{H}(F(\mu))$ falta calcular explicitamente as derivadas de primeira ordem $\delta_{n} m_{l}(\mu) \equiv \frac{\partial m_{l}(\mu)}{\partial \mu_{n}}, \delta_{n} k_{l}(\mu) \equiv \frac{\partial k_{l}(\mu)}{\partial \mu_{n}}$, e da segunda ordem $\delta_{i} \delta_{n} m_{l}(\mu) \equiv \frac{\partial^{2} m_{l}(\mu)}{\partial \mu_{i} \partial \mu_{n}}=\frac{\partial^{2} m_{l}(\mu)}{\partial \mu_{n} \partial \mu_{i}}, \delta_{i} \delta_{n} k_{l}(\mu) \equiv \frac{\partial^{2} k_{l}(\mu)}{\partial \mu_{i} \partial \mu_{n}}=\frac{\partial^{2} k_{l}(\mu)}{\partial \mu_{n} \partial \mu_{i}}, i=1,2, \ldots, n$.

Diferenciando $m_{n}$ em 2.28 com relação a $\mu_{n}$, obtém-se

$$
\delta_{n} m_{n}(\mu)=\frac{1}{1+\sum_{l=1}^{n-1}\left(\frac{y_{l}(\mu)}{y_{n}(\mu)}\right)^{2}}
$$


e diferenciando o resto das massas $\left(m_{l}\right)_{1}^{n-1}$ em 2.26

$$
\delta_{n} m_{l}(\mu)=\frac{\left(\frac{y_{l}(\mu)}{y_{n}(\mu)}\right)^{2}}{1+\sum_{l=1}^{n-1}\left(\frac{y_{l}(\mu)}{y_{n}(\mu)}\right)^{2}},
$$

com $l=1,2, \ldots, n-1$. Para calcular as derivadas das constantes de rigidez $k_{l}$ com relação a $\mu_{n}$, deve considerar-se que $k_{l}$ depende de $m(\mu)=\left(m_{1}(\mu), m_{2}(\mu), \ldots, m_{n}(\mu)\right)$. Assim,

onde

$$
\delta_{n} k_{l}(\mu)=\nabla k_{l}(m(\mu)) \cdot \frac{\partial \overrightarrow{m(} \mu)}{\partial \mu_{n}}
$$

$$
\nabla k_{l}(m(\mu))=\left(\frac{\partial k_{l}(\mu)}{\partial m_{1}}, \frac{\partial k_{l}(\mu)}{\partial m_{2}}, \ldots, \frac{\partial k_{l}(\mu)}{\partial m_{n}}\right)
$$

$$
\frac{\partial \overrightarrow{m(}(\mu)}{\partial \mu_{n}}=\left(\frac{\partial m_{1}(\mu)}{\partial \mu_{n}}, \frac{\partial m_{2}(\mu)}{\partial \mu_{n}}, \ldots, \frac{\partial m_{n}(\mu)}{\partial \mu_{n}}\right)
$$

Então,

$$
\delta_{n} k_{l}(\mu)=\sum_{j=1}^{n} \frac{\partial k_{l}(\mu)}{\partial m_{j}} \cdot \frac{\partial m_{j}(\mu)}{\partial \mu_{n}}
$$

onde

$$
\frac{\partial k_{l}(\mu)}{\partial m_{j}}= \begin{cases}\frac{m_{l-1}(\mu) b_{n-l+1}(\mu)}{2 \sqrt{m_{l-1}(\mu) m_{l}(\mu)}} & , \text { se } j=l \\ \frac{m_{l}(\mu) b_{n-l+1}(\mu)}{2 \sqrt{m_{l-1}(\mu) m_{l}(\mu)}} & , \text { se } j=l-1 \\ 0 & \text {, q.o.c. }\end{cases}
$$

para $j=1,2, \ldots, n, l=2,3, \ldots, n, \mathrm{e}$

$$
\frac{\partial k_{1}(\mu)}{\partial m_{j}}= \begin{cases}a_{n}(\mu)-\frac{\partial k_{2}(\mu)}{\partial m_{j}} & , \text { se } j=1 \\ -\frac{\partial k_{2}(\mu)}{\partial m_{j}} & , \text { se } j=2,3, \ldots, n\end{cases}
$$


Aqui $a_{n}(\mu)$ e $b_{n-l+1}(\mu)$ são os elementos da matriz de Jacobi $J(\mu)$ relativa ao sistema $(M(\mu), K(\mu))$.

Agora, para calcular as derivadas mistas $\left(\delta_{i} \delta_{n} m_{l}(\mu), \delta_{i} \delta_{n} k_{l}(\mu)\right)_{l=1}^{n}, i=1,2, \ldots, n-1$, primeiro são consideradas as eqs 5.20 e 5.21 para ser diferenciadas com relação a $\mu_{i}$, isto é,

$$
\delta_{i} \delta_{n} m_{n}(\mu)=\frac{-2 \sum_{l=1}^{n-1}\left(\frac{y_{l}(\mu)}{y_{n}^{3}(\mu)}\left(\delta_{i} y_{l}(\mu) y_{n}(\mu)-y_{l}(\mu) \delta_{i} y_{n}(\mu)\right)\right)}{\left[1+\sum_{l=1}^{n-1}\left(\frac{y_{l}(\mu)^{2}}{y_{n}(\mu)}\right)\right]^{2}}
$$

e

$$
\delta_{i} \delta_{n} m_{l}(\mu)=\delta_{i} \delta_{n} m_{n}(\mu)\left(\frac{y_{l}(\mu)}{y_{n}(\mu)}\right)^{2}+2 \frac{y_{l}(\mu)}{y_{n}^{3}(\mu)} \delta_{n} m_{n}(\mu)\left[\delta_{i} y_{l}(\mu) y_{n}(\mu)-y_{l}(\mu) \delta_{i} y_{n}(\mu)\right],
$$

para $i, l=1,2, \ldots, n-1$. Por outra parte, diferenciando a eq 5.25 com relação a $\mu_{i}$, obtém-se

$$
\delta_{i} \delta_{n} k_{l}(\mu)=\sum_{j=1}^{n}\left(\frac{\partial^{2} k_{l}(\mu)}{\partial \mu_{i} \partial m_{j}} \cdot \frac{\partial m_{j}(\mu)}{\partial \mu_{n}}+\frac{\partial k_{l}(\mu)}{\partial m_{j}} \cdot \frac{\partial^{2} m_{j}(\mu)}{\partial \mu_{i} \partial \mu_{n}}\right), i=1,2, \ldots, n-1
$$

onde,

$$
\begin{aligned}
& \frac{\partial^{2} k_{l}(\mu)}{\partial \mu_{i} \partial m_{j}}= \begin{cases} & {\left[2 m_{l-1}(\mu) m_{l}(\mu)\left[b_{n-l+1}(\mu) \delta_{i} m_{l-1}(\mu)+\delta_{i} b_{n-l+1}(\mu) m_{l-1}(\mu)\right]-\right.} \\
\left.m_{l-1}(\mu) b_{n-l+1}(\mu)\left[\delta_{i} m_{l-1}(\mu) m_{l}(\mu)+\delta_{i} m_{l}(\mu) m_{l-1}(\mu)\right]\right] \cdot \frac{1}{4\left[m_{l-1}(\mu) m_{l}(\mu)\right]^{3 / 2}}, & , \text { se } j=l \\
{\left[2 m_{l-1}(\mu) m_{l}(\mu)\left[b_{n-l+1}(\mu) \delta_{i} m_{l}(\mu)+\delta_{i} b_{n-l+1}(\mu) m_{l}(\mu)\right]-\right.} & \\
\left.m_{l}(\mu) b_{n-l+1}(\mu)\left[\delta_{i} m_{l-1}(\mu) m_{l}(\mu)+\delta_{i} m_{l}(\mu) m_{l-1}(\mu)\right]\right] \cdot \frac{1}{4\left[m_{l-1}(\mu) m_{l}(\mu)\right]^{3 / 2}} & , \text { se } j=l-\end{cases} \\
& 0 \text {, q.o.c. }
\end{aligned}
$$

$$
\frac{\partial^{2} k_{1}(\mu)}{\partial \mu_{i} \partial m_{j}}= \begin{cases}-\frac{\partial^{2} k_{2}(\mu)}{\partial \mu_{i} \partial m_{j}} & , \text { se } j=2,3, \ldots, n \\ \frac{\partial a_{n}(\mu)}{\partial \mu_{i}}-\frac{\partial^{2} k_{2}(\mu)}{\partial \mu_{i} \partial m_{j}} & , \text { se } j=1\end{cases}
$$


Finalmente, são calculadas $\delta_{n} \delta_{n} m_{l}(\mu) \equiv \frac{\partial^{2} m_{l}(\mu)}{\partial \mu_{n}(\mu) \partial \mu_{n}(\mu)}$, e $\delta_{n} \delta_{n} k_{l}(\mu) \equiv \frac{\partial^{2} k_{l}(\mu)}{\partial \mu_{n}(\mu) \partial \mu_{n}(\mu)}$. Claramente, diferenciando as eqs 5.20 e 5.21 com relação a $\mu_{n}$, obtém-se

$$
\delta_{n} \delta_{n} m_{l}(\mu)=0, l=1,2, \ldots, n
$$

Por outro lado,

$$
\begin{aligned}
\delta_{n} \delta_{n} k_{l}(\mu) & =\sum_{j=1}^{n}\left(\frac{\partial^{2} k_{l}(\mu)}{\partial \mu_{n} \partial \mu_{n}} \cdot \frac{\partial m_{j}(\mu)}{\partial \mu_{n}}+\frac{\partial k_{l}(\mu)}{\partial m_{j}} \cdot \frac{\partial^{2} m_{j}(\mu)}{\partial \mu_{n} \partial \mu_{n}}\right) \\
& =\sum_{j=1}^{n}\left(\frac{\partial^{2} k_{l}(\mu)}{\partial \mu_{n} \partial \mu_{n}} \cdot \frac{\partial m_{j}(\mu)}{\partial \mu_{n}}\right), l=1,2, \ldots, n,
\end{aligned}
$$

onde

$$
\frac{\partial^{2} k_{l}(\mu)}{\partial \mu_{n} \partial m_{j}}= \begin{cases}{\left[2 m_{l-1}(\mu) m_{l}(\mu) b_{n-l+1}(\mu) \delta_{n} m_{l-1}(\mu)-\right.} \\ \left.m_{l-1}(\mu) b_{n-l+1}(\mu)\left[\delta_{n} m_{l-1}(\mu) m_{l}(\mu)+\delta_{n} m_{l}(\mu) m_{l-1}(\mu)\right]\right] \frac{1}{4\left[m_{l-1}(\mu) m_{l}(\mu)\right]^{3 / 2}} & , \text { se } j=l \\ {\left[2 m_{l-1}(\mu) m_{l}(\mu) b_{n-l+1}(\mu) \delta_{n} m_{l}(\mu)-\right.} & \\ \left.m_{l}(\mu) b_{n-l+1}(\mu)\left[\delta_{n} m_{l-1}(\mu) m_{l}(\mu)+\delta_{n} m_{l}(\mu) m_{l-1}(\mu)\right]\right] \frac{1}{4\left[m_{l-1}(\mu) m_{l}(\mu)\right]^{3 / 2}} & , \text { se } j=l- \\ 0 & \text {, q.o.c. }\end{cases}
$$

para $l=2,3, \ldots, n, \mathrm{e}$

$$
\frac{\partial^{2} k_{1}(\mu)}{\partial \mu_{n} \partial m_{j}}=-\frac{\partial^{2} k_{2}(\mu)}{\partial \mu_{n} \partial m_{j}}
$$

Uma vez calculadas as derivadas de primeira e segunda ordem das massas e constantes de rigidez, com relação à massa total, o algoritmo 4.2 é adaptado adequadamente para calcular o vetor gradiente $F(\mu)$ e a matriz Hessiana $\mathcal{H}(F(\mu))$.

Agora, o algoritmo Box é aplicado para os mesmos valores iniciais gerados na Seção 5.2.1, isto é,

$$
\begin{aligned}
\mu_{l}^{v i} & =r_{l}\left(\bar{\lambda}_{l+1}-\bar{\lambda}_{l}-2 \varepsilon_{l}\right)+\bar{\lambda}_{l}+\varepsilon_{l}, l=1,2, \ldots, n-1, \\
\mathrm{e} & \\
\mu_{n}^{v i} & =r_{n}\left(b-a-2 \varepsilon_{n}\right)+\bar{\lambda}_{l}+\varepsilon_{n},
\end{aligned}
$$


Tabela 5.8:

\begin{tabular}{|c|c|c|c|c|c|}
\hline Teste & 1 & 2 & 3 & 4 & 5 \\
\hline Semente & -504 & -633 & -455 & -181 & -186 \\
\hline$d_{1}$ & 5.0 & 1000.0 & 1000.0 & 3.0 & 3.0 \\
\hline$d_{2}$ & 100.0 & 5.0 & 1000.0 & 100.0 & 5.0 \\
\hline$F\left(\mu^{*}\right)$ & $1.973449 \mathrm{e}-24$ & $2.290694 \mathrm{e}-23$ & $1.719045 \mathrm{e}-24$ & $6.415210 \mathrm{e}-24$ & $8.790579 \mathrm{e}-25$ \\
\hline$\left\|\nabla F\left(\mu^{*}\right)\right\|$ & $5.469392 \mathrm{e}-08$ & $2.058682 \mathrm{e}-08$ & $8.564058 \mathrm{e}-07$ & $1.897278 \mathrm{e}-08$ & $2.333149 \mathrm{e}-08$ \\
\hline $\begin{array}{c}N^{\circ} \text { Iterações } \\
\text { do Box }\end{array}$ & 45 & 64 & 45 & 197 & 60 \\
\hline Teste & 6 & 7 & 8 & 9 & 10 \\
\hline Semente & -458 & -634 & -901 & -727 & -565 \\
\hline$d_{1}$ & 1000.0 & 5.0 & 2.5 & 5.0 & 5.0 \\
\hline$d_{2}$ & 100.0 & 1000.0 & 100.0 & 100.0 & 1000.0 \\
\hline$F\left(\mu^{*}\right)$ & $4.422421 \mathrm{e}-24$ & $7.211806 \mathrm{e}-25$ & $7.130100 \mathrm{e}-24$ & $1.501836 \mathrm{e}-22$ & $4.263959 \mathrm{e}-25$ \\
\hline$\left\|\nabla F\left(\mu^{*}\right)\right\|$ & $9.847940 \mathrm{e}-10$ & $2.487210 \mathrm{e}-08$ & $2.607095 \mathrm{e}-08$ & $2.543513 \mathrm{e}-07$ & $4.279466 \mathrm{e}-08$ \\
\hline $\begin{array}{c}N^{\circ} \text { Iterações } \\
\text { do Box }\end{array}$ & 40 & 85 & 77 & 37 & 100 \\
\hline
\end{tabular}

onde $\varepsilon_{l}=\frac{\bar{\lambda}_{l+1}-\bar{\lambda}_{l}}{d_{1}}, \mathrm{e} \varepsilon_{n}=\frac{b-a}{d_{2}} \operatorname{com} d_{1}, d_{2}>2.0$.

A Tabela 5.8 mostra que em todos os casos o ponto $\mu^{*}$ foi atingido, confirmando numericamente que a estratégia multidimensional aplicada em sistemas massa-mola é eficiente e precisa.

No estudo anterior, não houve isolamentos de frequências, isto é, nenhum autovalor está dentro de uma banda de ressonância $\beta(c ; r)$ (os autovalores não foram perturbados). Agora, o problema, onde um ou mais autovalores estão dentro de $\beta(c ; r)$, será considerado.

Seja $(\bar{M}, \bar{K})$ o sistema massa-mola com autovalores $\left(\bar{\lambda}_{i}\right)_{1}^{n}$ tal que $\bar{\lambda}_{i} \in \beta(c ; r)$, com $i=$ $k, k+1, \ldots, m, 1<k \leq m<n$.

Antes de aplicar a estratégia multidimensional devem ser removidos os autovalores que ficam dentro de $\beta(c ; r)$. Isto é possível aplicando o procedimento descrito no Cap 2. Supondo que

$$
\bar{\lambda}_{i} \in\left\{\begin{array}{ll}
(c-r, c] & , i=k, k+1, \ldots, l \\
(c, c+r) & , i=l+1, l+2, \ldots, m
\end{array} .\right.
$$


Então os autovalores que estão dentro do intervalo $(c-r, c]$ podem ser removidos pela relação

$$
\lambda_{i}^{*}=\bar{\lambda}_{k-1}+\frac{\left(\bar{\lambda}_{i}-c+r\right)\left(c-r-\bar{\lambda}_{k-1}\right)}{r},
$$

com $i=k, k+1, \ldots, l$. Os autovalores que ficam no intervalo $(c, c+r)$ podem ser isolados por

$$
\lambda_{i}^{*}=c+r+\frac{\left(\bar{\lambda}_{m+1}-c-r\right)\left(\bar{\lambda}_{i}-c\right)}{r},
$$

para $i=l+1, l+2, \ldots, m$. Enquanto que, os autovalores fora da banda de ressonância são mantidos, isto é,

$$
\lambda_{i}^{*}=\bar{\lambda}_{i}, \forall i \neq j, j=k, k+1, \ldots, m .
$$

Observação: Note-se que, se é considerada a banda com centro $c=111.0$ e raio $r=14.0$, os autovalores $\bar{\lambda}_{5}=99.9$ e $\bar{\lambda}_{6}=123.3$ do sistema $(\bar{M}, \bar{K})$, listado na Tabela 5.5 , devem ser removidos. Usando o procedimento $5.38,5.39,5.40$, os autovalores isolados tornam-se $\lambda_{5}^{*}=74.48$ e $\lambda_{6}^{*}=148.37$. Claramente, o exemplo mostra que os autovalores que devem ser removidos podem ficar longe dos extremos da banda, o que pode prejudicar o desempenho da estratégia multidimensional. Isto é evitado introduzindo um fator $\delta>2.0$ no processo de isolamento de tal maneira que os autovalores removidos não sejam perturbados demais. Então,

$$
\lambda_{i}^{*}=\eta+\frac{\left(\bar{\lambda}_{i}-c+r\right)\left(c-r-\bar{\lambda}_{k-1}\right)}{r}, i=k, k+1, \ldots, l,
$$

$\operatorname{com} \eta=c-r-\frac{c-r-\bar{\lambda}_{k-1}}{\delta}, \mathrm{e}$

$$
\lambda_{i}^{*}=c+r+\frac{(\xi-c-r)\left(\bar{\lambda}_{i}-c\right)}{r}, i=l+1, l+2, \ldots, m,
$$

onde $\xi=c+r+\frac{\bar{\lambda}_{m+1}-c-r}{\delta}$.

Agora, os autovalores removidos, do exemplo anterior, tornam-se

$$
\begin{aligned}
& \lambda_{5}^{*}=94.74, \quad \lambda_{6}^{*}=127.33, \quad \text { se } \delta=10.0 \\
& \lambda_{5}^{*}=96.77, \quad \lambda_{6}^{*}=125.23, \quad \text { se } \delta=100.0 \\
& \lambda_{5}^{*}=96.97, \quad \lambda_{6}^{*}=125.02, \quad \text { se } \delta=1000.0 \text {. }
\end{aligned}
$$

$O$ fator $\delta$, serve também para controlar o espaçamento entre autovalores quando devem ser removidos mais de dois. Por exemplo, se $r=148.7$ e $c=25.5$ então $\bar{\lambda}_{6}=123.3, \bar{\lambda}_{7}=151.6$, $\bar{\lambda}_{8}=174.1 \in \beta(148.7 ; 25.5)$. Assim, para diferentes valores de $\delta$, os autovalores isolados ficam 


$$
\begin{aligned}
& \lambda_{6}^{*}=117.39, \quad \lambda_{7}^{*}=175.08, \quad \lambda_{8}^{*}=181.99, \quad \text { se } \delta=4.0 \\
& \lambda_{6}^{*}=120.29, \quad \lambda_{7}^{*}=174.64, \quad \lambda_{8}^{*}=178.09 \quad \text { se } \delta=8.0 \\
& \lambda_{6}^{*}=121.74, \quad \lambda_{7}^{*}=174.42, \quad \lambda_{8}^{*}=176.14 \quad \text { se } \delta=16.0 \\
& \lambda_{6}^{*}=122.47, \quad \lambda_{7}^{*}=174.31, \quad \lambda_{8}^{*}=175.17 \quad \text { se } \delta=32.0 \text {. }
\end{aligned}
$$

Depois da observação relativa ao procedimento de isolamento dos autovalores indesejados, são considerados alguns exemplos a fim de comprovar a eficiência da estratégia multidimensional em sistemas massa-mola submetidos a ressonância, isto é, quando um ou mais autovalores ficam dentro de uma banda $\beta(c ; r)$. Em todos os exemplos, o sistema-modelo terá 10 graus de liberdade como listado na Tabela 5.5. Além disso, o algoritmo Box utilizará o valor inicial gerado a partir da semente cujo valor é -458 . No primeiro exemplo, são considerados três testes diferentes quando um autovalor fica dentro de $\beta(c ; r)$. A Tabela 5.9 mostra os resultados da estratégia multidimensional quando é removido o autovalor $\bar{\lambda}_{2}$ de uma banda com centro igual a 26.0 e raio igual a $0.9,2.0$, e 4.0. Logo, é isolado um autovalor correspondente ao meio do espectro, $\bar{\lambda}_{6}$, e posteriormente o autovalor $\bar{\lambda}_{9}$, também com três bandas diferentes cada um. As Figuras 5.1,5.2, e 5.3 ilustram os sistemas recuperados depois de aplicar a estratégia multidimensional, os quais são comparados com o sistema inicial. Do lado esquerdo são ilustradas as massas, e do dereito as constantes de rigidez. Pode confirmar-se que os autovalores, correspondentes às frequências baixas, são mais sensível a perturbações, enquanto que as altas tem uma sensibilidade menor.

Agora, se dois autovalores devem ser isolados, os resultados são semelhantes ao primeiro exemplo. A Tabela 5.10 mostra os casos quando são isolados os autovalores $\bar{\lambda}_{2}, \bar{\lambda}_{3}$, depois dois, pertencente ao meio do espectro, $\bar{\lambda}_{5}, \bar{\lambda}_{6}$, e finalmente os autovalores extremos $\bar{\lambda}_{8}, \bar{\lambda}_{9}$. A sensibilidade é maior que no caso anterior, mesmo assim as diferentes perturbações feitas não causam grandes mudanças no re-projeto do sistema. Estes fatos são confirmados nas Figuras $5.4,5.5$, e 5.6 .

Finalmente, é considerado o caso correspondente ao isolamento de três autovalores $(30 \%$ do espectro perturbado). A Tabela 5.11, mostra os resultados obtidos depois de isolar os autovalores $\bar{\lambda}_{6}, \bar{\lambda}_{7}, \bar{\lambda}_{8}$ das bandas $\beta(148.7 ; 25.5), \beta(148.7 ; 29.0), \beta(148.7 ; 32.0)$, respectivamente. Obviamente, a sensibilidade é maior quando o número de autovalores perturbados é maior. Aliás, as maiores diferenças ficam na primeira massa do sistema modificado tal como é ilustrado na Figura 5.7.

Todos os exemplos confirmam que a estratégia multidimensional é eficiente, a qual permite identificar as mudanças que devem ser fietas nas propriedades físicas de um sistema massa-mola sujeito a ressonância. 
Tabela 5.9:

\begin{tabular}{|c|c|c|c|c|c|c|}
\hline Teste & 1 & 2 & 3 & 4 & 5 & 6 \\
\hline Semente & -458 & -458 & -458 & -458 & -458 & -458 \\
\hline$\delta$ & 1000.0 & 1000.0 & 1000.0 & 1000.0 & 1000.0 & 1000.0 \\
\hline$c$ & 26.0 & 26.0 & 26.0 & 120.0 & 120.0 & 120.0 \\
\hline$r$ & 0.9 & 2.0 & 4.0 & 3.4 & 6.0 & 12.0 \\
\hline $\bar{\lambda}_{i} \in \beta(c ; r)$ & $\bar{\lambda}_{2}=25.20$ & $\bar{\lambda}_{2}=25.20$ & $\bar{\lambda}_{2}=25.20$ & $\bar{\lambda}_{6}=123.30$ & $\bar{\lambda}_{6}=123.30$ & $\bar{\lambda}_{6}=123.30$ \\
\hline$\lambda_{i}^{*} \notin \beta(c ; r)$ & $\lambda_{2}^{*}=25.07$ & $\lambda_{2}^{*}=23.99$ & $\lambda_{2}^{*}=21.99$ & $\lambda_{6}^{*}=123.42$ & $\lambda_{6}^{*}=126.01$ & $\lambda_{6}^{*}=132.00$ \\
\hline$F\left(\mu^{*}\right)$ & $1.44 \mathrm{e}-04$ & $6.49 \mathrm{e}-03$ & 21.37 & $7.28 \mathrm{e}-06$ & $1.31 \mathrm{e}-02$ & 0.56 \\
\hline$\left\|\nabla F\left(\mu^{*}\right)\right\|$ & $7.49 \mathrm{e}-11$ & $2.96 \mathrm{e}-10$ & $4.32 \mathrm{e}-09$ & $6.70 \mathrm{e}-10$ & $2.55 \mathrm{e}-09$ & $6.17 \mathrm{e}-10$ \\
\hline $\begin{array}{c}N^{o} \text { Iterações } \\
\text { do Box }\end{array}$ & 38 & 94 & 59 & 56 & 47 & 50 \\
\hline Teste & 7 & 8 & 9 & & & \\
\hline Semente & -458 & -458 & -458 & & & \\
\hline$\delta$ & 1000.0 & 1000.0 & 1000.0 & & & \\
\hline$c$ & 204.5 & 204.5 & 204.5 & & & \\
\hline$r$ & 1.1 & 4.0 & 12.0 & & & \\
\hline $\bar{\lambda}_{i} \in \beta(c ; r)$ & $\bar{\lambda}_{9}=205.50$ & $\bar{\lambda}_{9}=205.50$ & $\bar{\lambda}_{9}=205.50$ & & & \\
\hline$\lambda_{i}^{*} \notin \beta(c ; r)$ & $\lambda_{9}^{*}=205.62$ & $\lambda_{9}^{*}=208.50$ & $\lambda_{9}^{*}=216.50$ & & & \\
\hline$F\left(\mu^{*}\right)$ & $7.07 \mathrm{e}-07$ & $5.18 \mathrm{e}-02$ & $8.06 \mathrm{e}-03$ & & & \\
\hline$\left\|\nabla F\left(\mu^{*}\right)\right\|$ & $1.14 \mathrm{e}-09$ & $2.04 \mathrm{e}-09$ & $1.09 \mathrm{e}-09$ & & & \\
\hline $\begin{array}{c}N^{o} \text { Iterações } \\
\text { do Box }\end{array}$ & 67 & 62 & 64 & & & \\
\hline
\end{tabular}



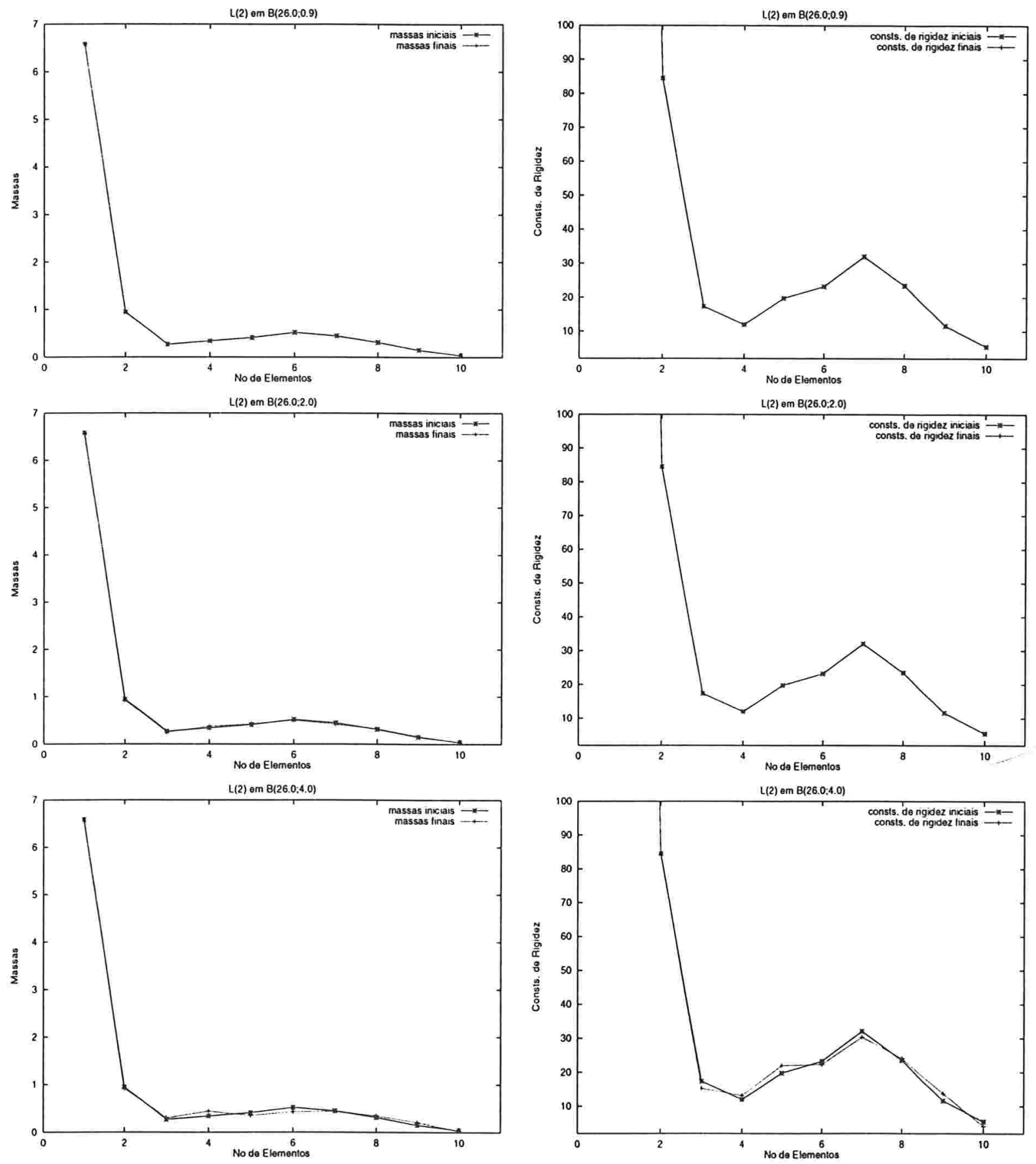

Figura 5.1: Comparação entre o sistema inicial e o final quando é isolado o segundo autovalor das bandas $\beta(26.0 ; 0.9), \beta(26.0 ; 2.0)$, e $\beta(26.0 ; 4.0)$. 

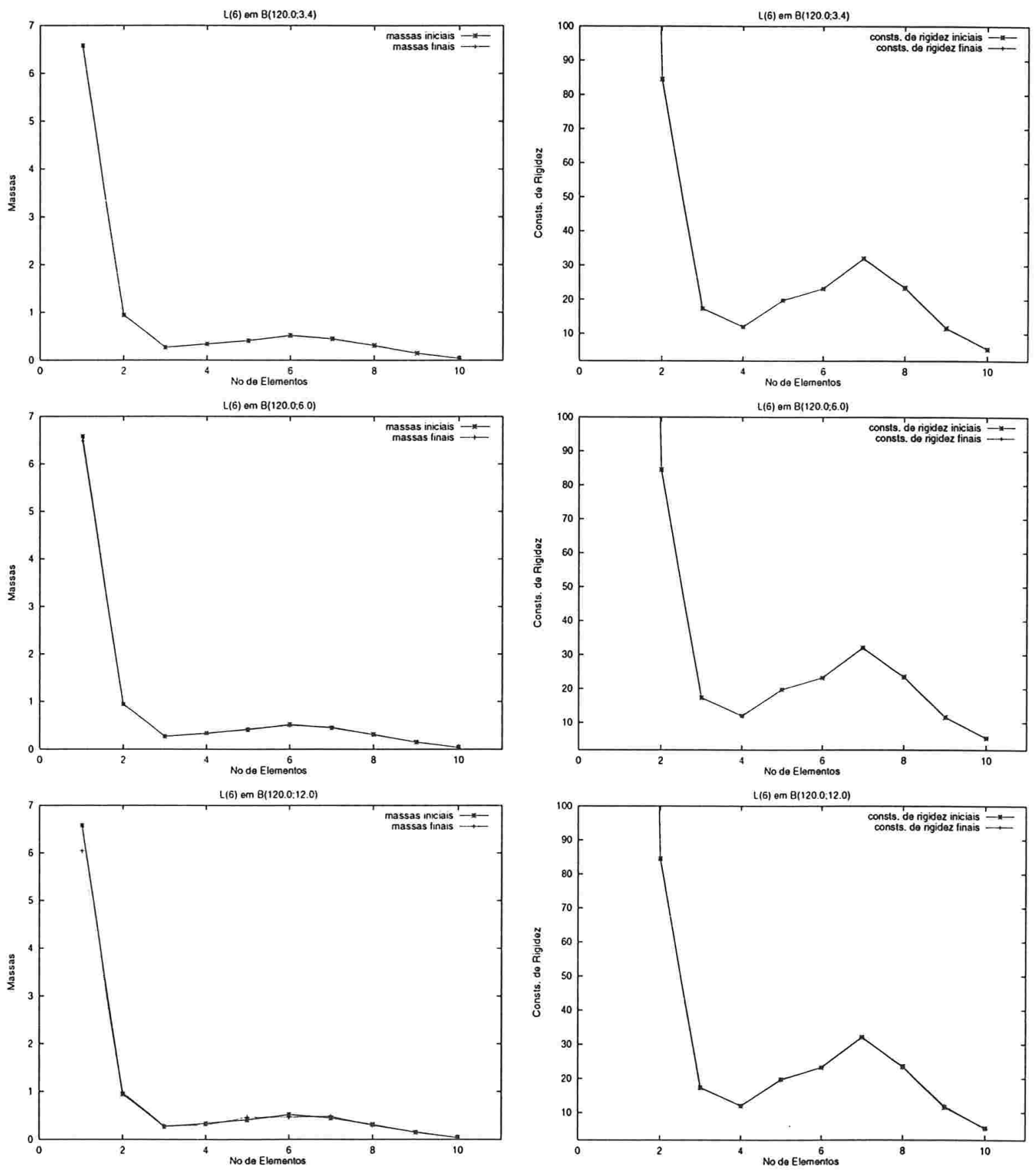

Figura 5.2: Comparação entre o sistema inicial e o final quando é isolado o sexto autovalor das bandas $\beta(120.0 ; 3.4), \beta(120.0 ; 6.0)$, e $\beta(120.0 ; 12.0)$. 

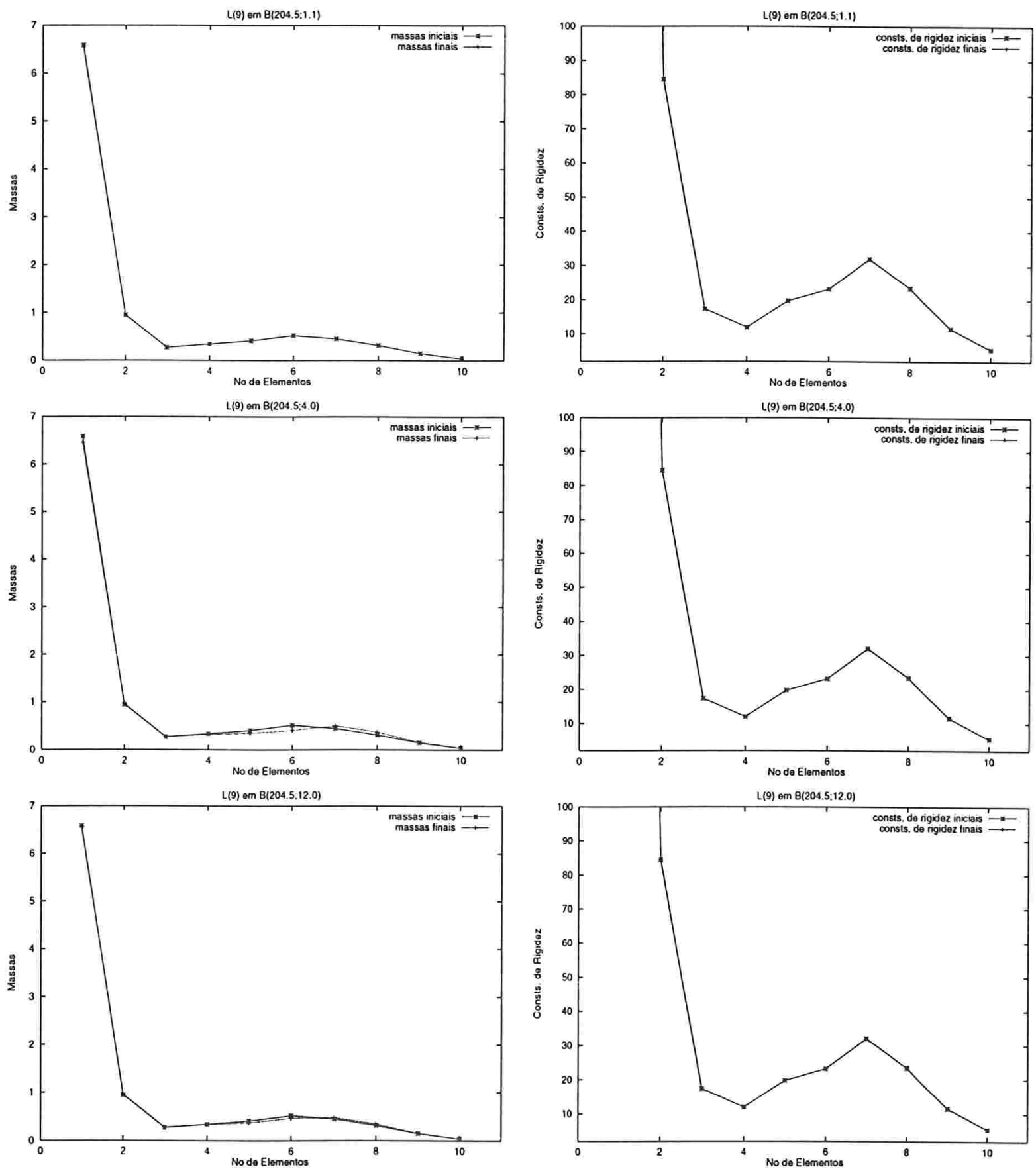

Figura 5.3: Comparação entre o sistema inicial e o final quando é isolado o nono autovalor das bandas $\beta(204.5 ; 1.1), \beta(204.5 ; 4.0)$, e $\beta(204.5 ; 12.0)$. 
Tabela 5.10:

\begin{tabular}{|c|c|c|c|c|c|c|}
\hline Teste & 1 & 2 & 3 & 4 & 5 & 6 \\
\hline Semente & -458 & -458 & -458 & -458 & -458 & -458 \\
\hline$\delta$ & 1000.0 & 1000.0 & 1000.0 & 1000.0 & 1000.0 & 1000.0 \\
\hline$c$ & 37.55 & 37.55 & 37.55 & 111.0 & 111.0 & 111.0 \\
\hline$r$ & 12.4 & 12.4 & 12.4 & 14.0 & 20.0 & 25.0 \\
\hline $\bar{\lambda}_{i} \in \beta(c ; r)$ & $\begin{array}{c}\bar{\lambda}_{2}=25.2 \\
\bar{\lambda}_{3}=49.90\end{array}$ & $\begin{array}{l}\bar{\lambda}_{2}=25.2 \\
\bar{\lambda}_{3}=49.90\end{array}$ & $\begin{array}{l}\bar{\lambda}_{2}=25.2 \\
\bar{\lambda}_{3}=49.90\end{array}$ & $\begin{array}{c}\bar{\lambda}_{5}=99.9 \\
\bar{\lambda}_{6}=123.3\end{array}$ & $\begin{array}{c}\bar{\lambda}_{5}=99.9 \\
\bar{\lambda}_{6}=123.3\end{array}$ & $\begin{array}{c}\bar{\lambda}_{5}=99.9 \\
\bar{\lambda}_{6}=123.3\end{array}$ \\
\hline$\lambda_{i}^{*} \notin \beta(c ; r)$ & $\begin{array}{l}\lambda_{2}^{*}=25.12 \\
\lambda_{3}^{*}=49.96\end{array}$ & $\begin{array}{l}\lambda_{2}^{*}=23.53 \\
\lambda_{3}^{*}=51.56\end{array}$ & $\begin{array}{l}\lambda_{2}^{\star}=22.53 \\
\lambda_{3}^{*}=52.56\end{array}$ & $\begin{array}{c}\lambda_{5}^{*}=96.97 \\
\lambda_{6}^{*}=125.02\end{array}$ & $\begin{array}{c}\lambda_{5}^{*}=90.98 \\
\lambda_{6}^{*}=131.01\end{array}$ & $\begin{array}{c}\lambda_{5}^{*}=85.99 \\
\lambda_{6}^{*}=136.00\end{array}$ \\
\hline$F\left(\mu^{*}\right)$ & $1.51 \mathrm{e}-04$ & 0.26 & 1.64 & $3.58 \mathrm{e}-02$ & 0.41 & 3.11 \\
\hline$\left\|\nabla F\left(\mu^{*}\right)\right\|$ & $3.32 \mathrm{e}-09$ & $4.73 \mathrm{e}-11$ & $1.71 \mathrm{e}-07$ & $6.28 \mathrm{e}-10$ & $6.31 \mathrm{e}-10$ & $4.16 \mathrm{e}-11$ \\
\hline $\begin{array}{c}N^{o} \text { Iterações } \\
\text { do Box }\end{array}$ & 53 & 94 & 82 & 72 & 65 & 45 \\
\hline Teste & 7 & 8 & 9 & & & \\
\hline Semente & -458 & -458 & -458 & & & \\
\hline$\delta$ & 1000.0 & 1000.0 & 1000.0 & & & \\
\hline$c$ & 111.0 & 111.0 & 111.0 & & & \\
\hline$r$ & 14.0 & 20.0 & 25.0 & & & \\
\hline $\bar{\lambda}_{i}$ & $\begin{array}{c}\bar{\lambda}_{5}=99.9 \\
\bar{\lambda}_{6}=123.30\end{array}$ & $\begin{array}{c}\bar{\lambda}_{5}=99.9 \\
\bar{\lambda}_{6}=123.30\end{array}$ & $\begin{array}{c}\bar{\lambda}_{5}=99.9 \\
\bar{\lambda}_{6}=123.30\end{array}$ & & & \\
\hline$\lambda_{i}^{*}$ & $\begin{array}{c}\lambda_{5}^{*}=96.97 \\
\lambda_{6}^{*}=125.02\end{array}$ & $\begin{array}{c}\lambda_{5}^{*}=90.98 \\
\lambda_{6}^{*}=131.01\end{array}$ & $\begin{array}{c}\lambda_{5}^{*}=85.99 \\
\lambda_{6}^{*}=136.00\end{array}$ & & & \\
\hline$F\left(\mu^{*}\right)$ & $3.58 \mathrm{e}-02$ & 0.41 & 3.11 & & & \\
\hline$\left\|\nabla F\left(\mu^{*}\right)\right\|$ & $6.28 \mathrm{e}-10$ & $6.31 \mathrm{e}-10$ & $4.16 \mathrm{e}-11$ & & & \\
\hline $\begin{array}{c}N^{\circ} \text { Iterações } \\
\text { do Box }\end{array}$ & 72 & 65 & 45 & & & \\
\hline
\end{tabular}



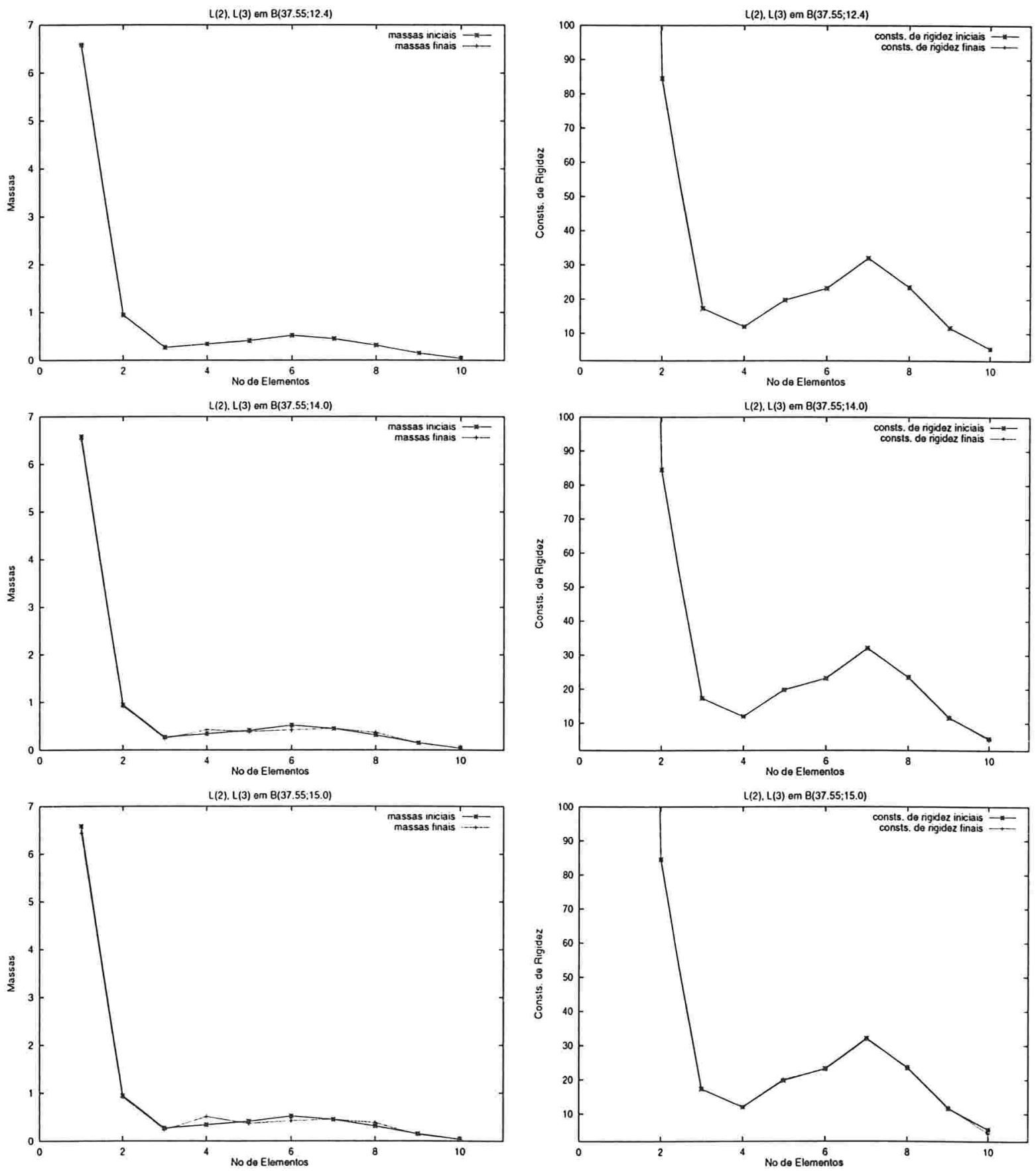

Figura 5.4: Comparação entre o sistema inicial e o final quando são isolados o segundo e terceiro autovalor das bandas $\beta(37.55 ; 12.4), \beta(37.55 ; 14.0)$, e $\beta(37.55 ; 15.0)$. 

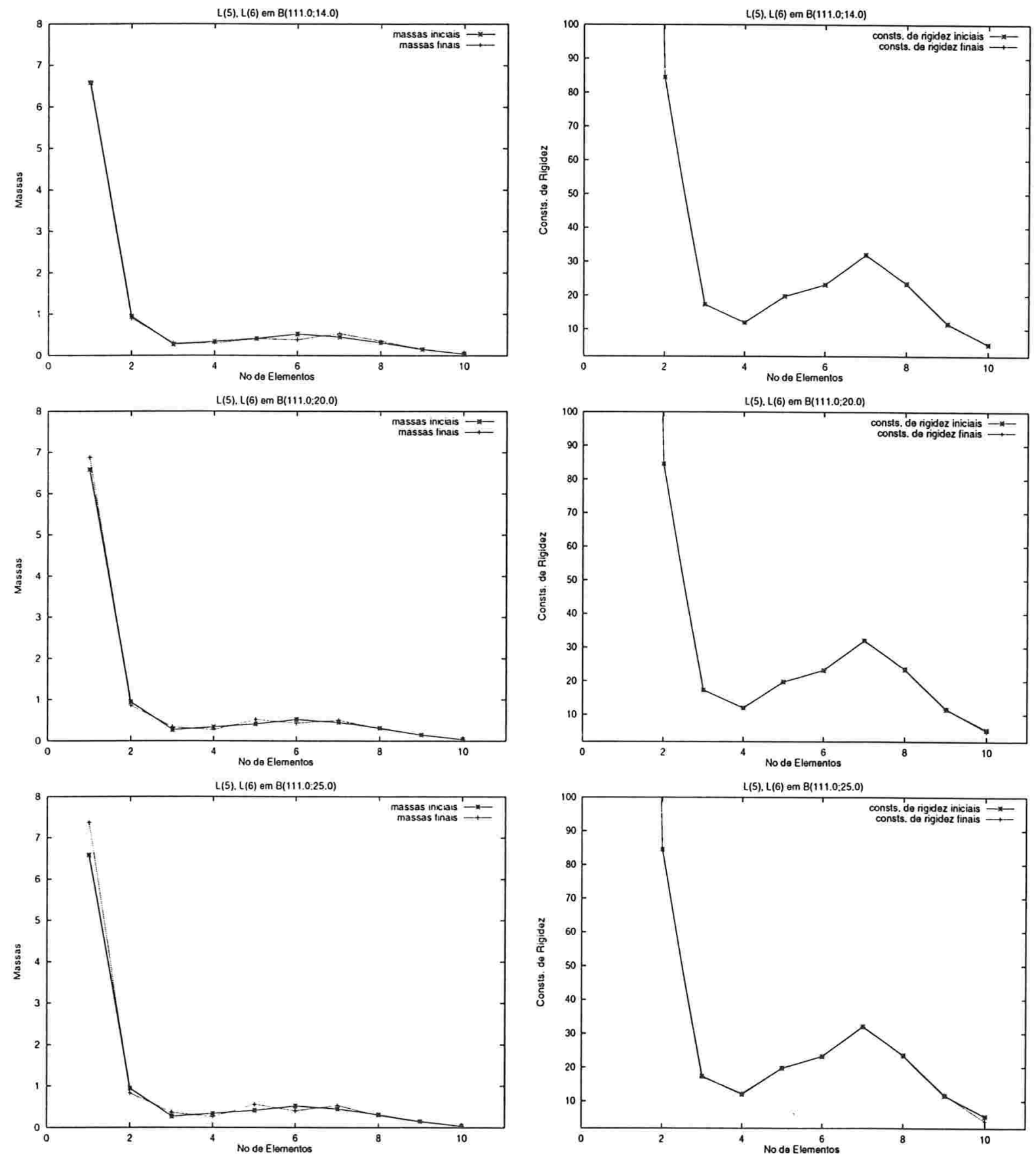

Figura 5.5: Comparação entre o sistema inicial e o final quando são isolados o quinto e sexto autovalor das bandas $\beta(111.0 ; 14.0), \beta(111.0 ; 20.0)$, e $\beta(111.0 ; 25.0)$. 

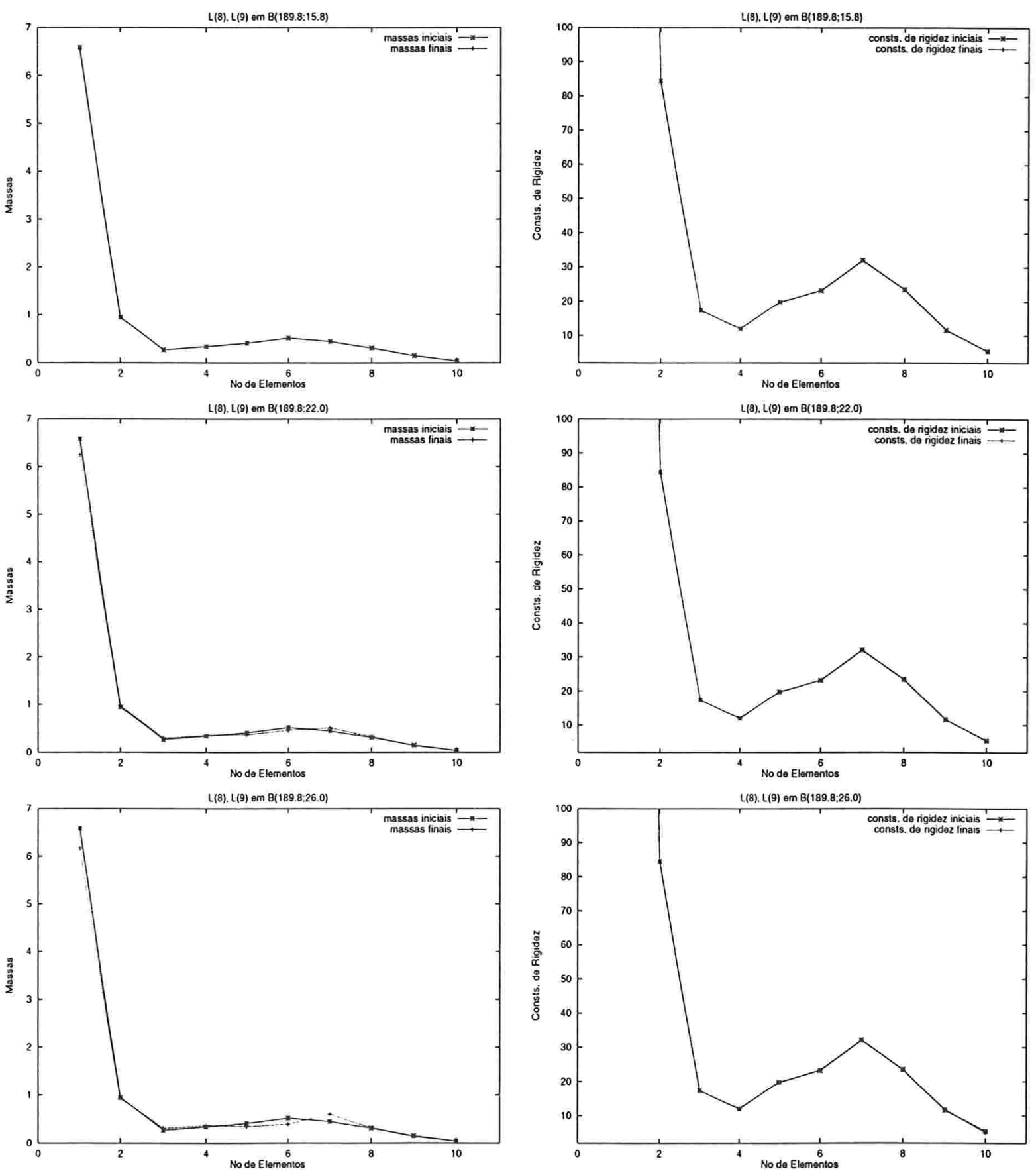

Figura 5.6: Comparação entre o sistema inicial e o final quando são isolados o oitavo e nono autovalor das bandas $3(189.8 ; 15.8), \beta(189.8 ; 22.0)$, e $\beta(189.8 ; 26.0)$. 

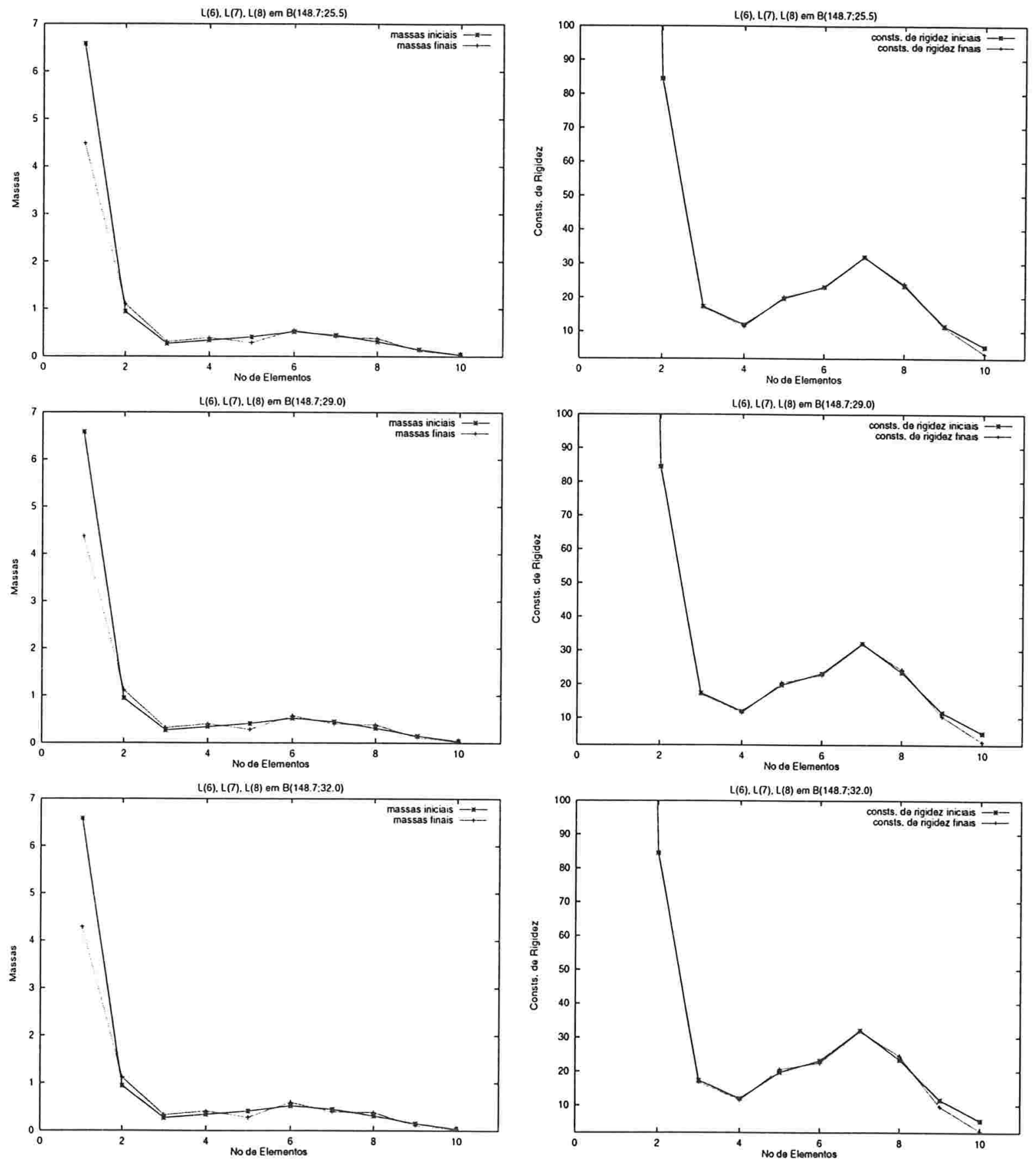

Figura 5.7: Comparação entre o sistema inicial e o final quando são isolados o sétimo, oitavo $e$ nono autovalor das bandas $\beta(148.7 ; 25.5), \beta(148.7 ; 29.0)$, e $\beta(148.7 ; 32.0)$. 
Tabela 5.11:

\begin{tabular}{c||ccc}
\hline \hline Teste & 1 & 2 & 3 \\
\hline Semente & -458 & -458 & -458 \\
\hline$\delta$ & 8.0 & 8.0 & 8.0 \\
\hline$c$ & 148.7 & 148.7 & 148.7 \\
\hline$r$ & 25.5 & 29.0 & 32.0 \\
\hline \multirow{4}{*}{$\bar{\lambda}_{i} \in 3(c ; r)$} & $\bar{\lambda}_{6}=123.30$ & $\bar{\lambda}_{6}=123.30$ & $\bar{\lambda}_{6}=123.30$ \\
& $\bar{\lambda}_{7}=151.60$ & $\bar{\lambda}_{7}=151.60$ & $\bar{\lambda}_{7}=151.60$ \\
& $\bar{\lambda}_{8}=174.10$ & $\bar{\lambda}_{8}=174.10$ & $\bar{\lambda}_{8}=174.10$ \\
\hline \multirow{2}{*}{$\lambda_{i}^{*} \notin 3(c ; r)$} & $\lambda_{6}^{*}=120.29$ & $\lambda_{6}^{*}=117.53$ & $\lambda_{6}^{*}=115.03$ \\
& $\lambda_{7}^{*}=174.64$ & $\lambda_{7}^{*}=178.04$ & $\lambda_{7}^{*}=180.98$ \\
& $\lambda_{8}^{*}=178.09$ & $\lambda_{8}^{*}=180.74$ & $\lambda_{8}^{*}=183.16$ \\
\hline F $\left(\mu^{\times}\right)$ & 9.79 & 14.71 & 21.27 \\
\hline$\left\|\nabla F\left(\mu^{*}\right)\right\|$ & $1.06 \mathrm{e}-10$ & $3.77 \mathrm{e}-05$ & $8.35 \mathrm{e}-09$ \\
\hline$N^{o}$ Iteraçoes & 63 & 56 & 64 \\
do Box & & & \\
\hline \hline
\end{tabular}




\section{Conclusões}

Neste trabalho foi desenvolvida uma metodologia eficiente e robusta para resolver o problema de isolamento de frequências IFSMM, pertencente a um amplo e atual campo de pesquisa tais como problemas de identificação, projeto estrutural e otimização. Tal como foi discutido no presente trabalho, o problema de isolamento de frequências consiste em isolar as frequências de um sistema estrutural que estão dentro de uma ou mais bandas de ressonância, gerando um novo espectro de frequências, e a partir deste, reconstruir um novo sistema minimizando o impacto de re-projeto relativo ao sistema inicial. Aqui é proposto um método numérico inverso de autovalor para resolver o problema IFSMM. A fim de minimizar o impacto de re-projeto a escolha entre as soluções inversas é aquela que minimiza as mudanças nas propriedades estruturais. Para tal, um espectro auxiliar é necessário. Um sistema massa-mola com $n$ graus de liberdade é usado para demonstrar a viabilidade e potencial da técnica.

Primero foi desenvolvida uma estratégia para remover as frequências de uma banda previamente especificada. Uma vez removidas as frequências indesejadas foi proposto um algoritmo de busca unidimensional do espectro auxiliar para minimizar o impacto de re-projeto do sistema. Quando o sistema-modelo é um sistema massa-mola, o espectro auxilar é chamado espectro intercalado, denotado por $\mu=\left(\mu_{1}, \mu_{2}, \ldots, \mu_{n-1}\right)$. Os resultados numéricos validam a estratégia unidimensional, mas também indicam suas limitações, motivando o desenvolvimento de uma técnica mais sofisticada e eficiente do ponto de vista computacional.

Uma generalização da estrátegia unidimensional consiste na busca multidimensional do espectro intercalado desconhecido. Neste caso, é definida a função objetivo

$$
F(\mu)=\sum_{j=1}^{n}\left(m_{j}(\mu)-\bar{m}_{j}\right)^{2}+\sum_{j=1}^{n}\left(k_{j}(\mu)-\bar{k}_{j}\right)^{2},
$$

Então o problema torna-se 


$$
\begin{aligned}
\text { Minimizar } & F(\mu) \\
\text { sujeito a : } & \lambda_{j}^{*}<\mu_{j}<\lambda_{j+1}^{*}
\end{aligned}
$$

O método de minimização Box, desenvolvido por Friedlander, Martínez e Santos [17], o qual minimiza uma função de várias variáveis com restrições de canalização, foi usado para resolver o problema variacional descrito acima. Na implementação deste método, é necessário determinar, com exatidão numérica, o vetor gradiente e a matriz Hessiana da função objetivo $F(\mu)$. Com esse fim, foi desenvolvida uma análise completa de sensibilidade em sistemas massa-mola com relação ao espectro intercalado $\mu$. Além disso, como a reconstrução de um sistema massa-mola é precedida pela reconstrução de uma matriz de Jacobi $J(\mu)$, um método numérico direto que calcula a derivada de $J(\mu)$, com relação a $\mu$, é proposto. Este é formulado como uma extensão do clássico método de Lanczos para o problema inverso de autovalor de uma matriz de Jacobi. $\mathrm{O}$ método, chamado $\delta$-Lanczos, calcula a derivada exata exceto por erros de arredondamento. Alguns exemplos são discutidos em ordem a demonstrar a eficiência, consistência, e exatidão do método. Por comparação com o esquema de diferenças centradas, o método $\delta$-Lanczos é mais vantajoso em termos de exatidão e custo computacional. Uma extensão do método $\delta$-Lanczos para derivadas de ordem mais alta é proposto para determinar a matriz Hessiana de $J(\mu)$, e portanto, da função objetivo $F(\mu)$.

Uma vez feito o estudo de sensibilidade de matrizes de Jacobi e sistemas massa-mola, são apresentados os resultados numéricos da estratégia multidimensional. A princípio, a massa total $m_{T}$ do sistema foi mantida constante, mas, experimentos numéricos sugeriram tornar $m_{T}$ uma variável independente. Este fato, permitiu relaxar o processo de minimização na busca do mínimo. Os resultados numéricos obtidos são excelentes e confirmam numericamente a eficiência e precisão da estratégia multidimensional. Assim, a estratégia permite identificar as mudanças que devem ser feitas nas propriedades físicas de um sistema massa-mola sujeito a ressonância.

Certamente os métodos e resultados obtidos neste trabalho podem ser melhorados e estendidos. Por exemplo, um trabalho de pesquisa futuro é obter métodos mais eficientes para determinar a sensibilidade de uma matriz de Jacobi, isto é, aproveitar métodos de reconstrução para matrizes deste tipo com um custo computacional menor. Além disso, o comportamento particular das derivadas dos parâmetros físicos em sistemas massa-mola, com relação ao espectro intercalado, sugere que através de um estudo de sensibilidade podem ser determinadas as mudanças que devem ser feitas no re-projeto do sistema.

O procedimento para isolar as frequências de uma banda proposto no Capítulo 2, é uma possibilidade que pode ser melhorada. Por exemplo, no Capítulo 5 , foi incluido um fator adicional $\varepsilon_{l}$ que permite controlar grandes perturbações que podem acontecer no processo de isolamento. Isto sugere que pode ser desenvolvido um procedimento mais eficiente na escolha de $\varepsilon_{l}$, ou talvez 
desenvolver um outro procedimento de isolamento que permita obter resultados ainda melhores.

O modelo usado neste trabalho corresponde ao modelo discreto mais simples possível para uma viga vibrando em movimento logitudinal. Barcilon em [1], [3], e Gladwell em [22], [25], [30] desenvolveram alguns métodos de reconstrução de um modelo discreto para a vibração transversal de uma viga. Aqui dois espectros auxiliares são necessários. Estes fatos podem ser aproveitados para aplicar, de uma maneira adequada, a estratégia multidimesional em sistemas de ordem mais alta. Além disso, uma aplicação da estratégia multidimensional pode ser aplicada em sistemas contínuos previamente discretizados via diferenças finitas. Atualmente Gladwell, em [23], [27], [28], está desenvolvendo métodos de reconstrução de sistemas massa-mola via elementos finitos, mas a falta de unicidade dos métodos obtidos ainda não permitem visualizar um caminho claro de pesquisa usando esta via de discretização.

Existem outros problemas de aplicação, tais como a deteç̧ão de falhas, onde pode ser aplicado o método inverso de autovalor proposto neste trabalho. Esta e todas as idéias anteriores fazem parte das linhas de pesquisa no futuro. 


\section{Apêndice A}

\section{Algoritmo tipo região de confiança para minimização com restrições de canalização Box}

Considerar o problema

$$
\begin{aligned}
\text { Minimizar } & F(x) \\
\text { sujeito a : } & l \leq x \leq u,
\end{aligned}
$$

onde $F: \Re^{n} \longrightarrow \Re$ é diferenciável em um conjunto factível. A.R. Conn et all [9] mostraram que praticamente todos os problemas de programação não-linear de grande porte pode ser resolvido eficientemente usando técnicas de Lagrangiano aumentado, sempre que seja usado um bom método para resolver A.2.

O algoritmo Box é um algoritmo do tipo região de confiança para resolver A.2. Em cada iteração do algoritmo, considera-se o problema de minimizar uma função quadrática (não necessariamente convexa) em uma caixa que consiste da interseção da caixa original $l<x<u$ com a região de confiança.

Usualmente em métodos modernos do tipo região de confiança, não é necessário resolver exatamente o subproblema para obter convergência global do algoritmo principal (Ver [9], [57], [54]). Em vez, uma condição suave que relaciona o valor objetivo da quadrática à solução de um problema auxiliar muito simples é suficiente para provar que todo ponto de acumulação é estacionário.

O gradiente de $F$ será denotado por $G(x)$. O símbolo $\|\cdot\|$ denota a norma-2 de vetores 
A. Algoritmo tipo região de confiança para minimização com restrições de canalização Box

e matrizes, e $\|\cdot\|_{\sharp}$ denotará uma norma arbitrária em $\Re^{n}$ e a correspondente norma matricial subordinada. Sejam $\alpha, \Delta_{\min }$, e $\theta$ tal que $\alpha \in(0,1), \Delta_{\min }>0$, e $\theta \in(0,1]$. No começo o algoritmo Box tem um ponto factível $x_{0}$, uma matriz simétrica $B_{0}$ de $n \times n$ (a aproximação da Hessiana), uma matriz não singular $D_{0}$ (matriz escala) de $n \times n$, e um raio $\Delta^{0} \geq \Delta_{\text {min }}$. O papel das matrizes escalas é permitir a possibilidade do ajuste das variáveis que diferem muito em maginitude. Dado um $x_{k}$ factível, $B_{k}$ simétrica, $D_{k}$ não singular, e $\Delta^{k} \geq \Delta_{m i n}$, os passos para obter $x_{k+1}$ e $\Delta_{k}$ são dados pelo seguinte

ALGORITMO Box:

Passo 1 (Definir o raio inicial da região de confiança e calcular uma estimativa superior of $\left.\lambda_{n}\left(B_{k}\right)\right)$. Definir

$$
\Delta \leftarrow \Delta^{k}
$$

Calcular $M_{k}>0$ tal que $\lambda_{n}\left(B_{k}\right) \leq M_{k}$, onde $\lambda_{1}\left(B_{k}\right)<\lambda_{2}\left(B_{k}\right)<\cdots<\lambda_{n}\left(B_{k}\right)$ são os autovalores de $B_{k}$.

Passo 2 (Resolver o subproblema "fácil"). Calcular a solução global $z_{k}^{Q}(\Delta)$ de

$$
\begin{aligned}
\text { Minimizar } & Q_{k}(z) \equiv \frac{1}{2} M_{k}\|z\|^{2}+G_{k}^{T} z, \\
\text { sujeito a : } & l \leq x_{k}+z \leq u \\
& \left\|D_{k} z\right\|_{\sharp} \leq \Delta,
\end{aligned}
$$

onde $G_{k}=G\left(x_{k}\right)$. Se $Q_{k}\left(z_{k}^{Q}(\Delta)\right)=0$, parar.

Passo 3 (Calcular o passo provável). Calcular $\bar{z}_{k}(\Delta)$ tal que

$$
\begin{aligned}
& \psi\left(\bar{z}_{k}(\Delta)\right) \leq \theta Q_{k}\left(z_{k}^{Q}(\Delta)\right), \\
& l \leq x_{k}+\bar{z}_{k}(\Delta) \leq u, \\
& \left\|D_{k} \bar{z}_{k}(\Delta)\right\|_{\sharp} \leq \Delta,
\end{aligned}
$$

onde $\psi(z)=\frac{1}{2} z^{T} B_{k} z+G_{k}^{T} z$ para todo $z \in \Re^{n}$. Observar que $z_{k}(\Delta)$ satisfazendo A.3- A.4 existe, já que $z_{k}^{Q}(\Delta)$ é uma escolha admissível.

Passo \& (Teste de parada). Se

$$
F\left(x_{k}+\bar{z}_{k}(\Delta)\right) \leq F\left(x_{k}\right)+\alpha \psi_{k}\left(\bar{z}_{k}(\Delta)\right),
$$


A. Algoritmo tipo região de confiança para minimização com restrições de canalização Box

então definir $z_{k}=\bar{z}_{k}(\Delta), x_{k+1}=x_{k}+z_{k}, \Delta_{k}=\Delta$ e parar. Senão, sustituir $\Delta$ por $\Delta_{\text {novo }}$, onde

$$
\Delta_{\text {novo }} \in\left[\sigma_{1}\left\|D_{k} \bar{z}_{k}(\Delta)\right\|_{\sharp}, \sigma_{2} \Delta\right]
$$

onde $\sigma_{1}, \sigma_{2}$ são tais que $0<\sigma_{1} \leq \sigma_{2}<1$, e ir ao Passo 2 .

Lema A. 1 : O algoritmo Box para no passo $2\left(Q_{k}\left(z_{k}^{Q}(\Delta)\right)=0\right)$ se e somente se $x_{k}$ é um ponto Kuhn-Tucker (estacionário) do problema A.1, A.2.

Teorema A. 1 O algoritmo Box é bem definido. Isto é, se o processo não para no Passo 2 (com $Q_{k}\left(z_{k}^{Q}(\Delta)\right)=0$ ), então $x_{k+1}$ podem calcular-se repetindo os Passos 2-4 em um número finito de vezes.

Observação: Normalmente sugere-se que a norma infinito $\|\cdot\|_{\infty}$ seja a escolha mais natural para $\|\cdot\|_{\sharp}$.

No seguinte teorema prova-se a convergência global do algoritmo Box, onde introduze-se a notação $\lim _{k \in K}$ para denotar o limite quando $k \rightarrow \infty$ para $k$ pertencente a um conjunto infinito de indices $K$ subconjunto dos números naturais.

Teorema A. 2 Supor que $\left\{x_{k}\right\}$ é uma sequência gerada pelo algoritmo Box, $K_{1}$ é um conjunto infinito de indices tal que $\lim _{k \in K_{1}} x_{k}=x_{*}$, e $M_{k},\left\|D_{k}\right\|_{\sharp},\left\|D_{k}^{-1}\right\|_{\sharp}, e\left|\lambda_{1}\left(B_{k}\right)\right|$ são limitados por $k \in K_{1}$. Então $x_{*}$ é um ponto estacionário (Kuhn-Tucker) de A.1, A.2. 


\section{Referências Bibliográficas}

[1] V. Barcilon, On the multiplicity of solutions of the inverse problem for a vibrating beam, SIAM J. Appl. Math. 37 (1978), 605-613.

[2] _ On multiplicity of solutions of the inverse problems for a vibrating beam, SIAM J. Appl. Math. 37 (1979), 119-127.

[3] _ Inverse problems for the vibrating beam in the free-clamped configuration, Phil. Trans. Roy. Soc. Lond. A 304 (1982), 211-252.

[4] _ A two-dimensional inverse eigenvalue problem, Inverse Problems 6 (1990), 11-20.

[5] F.W. Biegler-Köning, Construction of band matrices from spectral data, Lin. Alg. Appl. 40 (1981), 79-84.

[6] D. Boley e G.H. Golub, A survey of matrix inverse eigenvalue problem, Inverse Problems 3 (1987), 595-622.

[7] D.L. Boley e G.H. Golub, Inverse eigenvalue problems for band matrices, Lectures Notes on Mathematics, Numerical Analysis (Berlin: Springer) (1977).

[8] M.T. Chu, Inverse eigenvalue problems, SIAM Rev. 40 (1998), no. 1, 1-39.

[9] A.R. Conn, N.I.M. Gould e Ph.L. Toint, Global convergence of a class of trust-region algorithms for optimization with simple bounds, SIAM Journal on Numerical Analysis $\mathbf{2 5}$ (1988), 433-460.

[10] H. Dai, About an inverse eigenvalue problem arising in vibration analysis, RAIRO Modél. Math. Anal. Numér. 29 (1995), 421-424.

[11] B.N. Datta, S. Elhay e Y.M. Ram, Orthogonality and partial pole assignment for the symmetric definite quadratic pencil, Lin. Alg. Appl. 257 (1997), 29-48.

[12] C. Davini, F. Gatti e A. Morassi, Damage analysis of steel beam, Meccanica 28 (1993), $27-37$. 
[13] C. Davini, A. Morassi e N. Rovere, Modal analysis bar tests and comments on the sensitivity of an procedure, Sound Vib. 179 (1995), 513-.527.

[14] C. de Boor e G.H. Golub, The numerically stable reconstruction of a jacobi matrix from spectral data, Lin. Alg. Appl. 21 (1978), 245-260.

[15] J.C. Egaña, L.C. Santos e N. Kuhl, Sensitivity analysis of jacobi matrices, Submitted (1999).

[16] _ A strategy for frequencies isolation in discrete dynamical systems, Proceedings VI Pan American Congress of Applied Mechanics, Rio de Janeiro 6 (1999), 243-246.

[17] A. Friedlander, J.M. Martínez e S.A. Santos, A new trust region algorithm for bound constrained minimization, Appl. Math. Optim 30 (1994), 235-266.

[18] F.P. Gantmakher e M.G. Krein, Oscillation matrices and kernels and small vibrations of mechanical systems, State Publishing House for Technical-Theorical, Literature, MoscowLeningrad, USSR (Translation: US Atomic Energy Commission, Washington DC, 1961) (1950).

[19] G.M. Gladwell, Inverse problems in vibrations, Appl. Mech. Rev. 39 (1986), no. 7.

[20] _ Inverse problems in vibrations-2, Appl. Mech. Rev. 49 (1996), no. 10.

[21] G.M. Gladwell e B.R. Zhu, Inverse problems for multidimensional vibrating systems, Proc. R. Soc. Lond A 439 (1992), 511-530.

[22] G.M.L. Gladwell, The approximation of uniform beams in transverse vibration by sets of masses elastically connected, Proc. 4th U.S. National Congress of Applied Mechanics, New York: Am. Soc. Mech. Eng. (1962), 169-176.

[23] _ The inverse problem for the beam, Proc. Roy. Soc. Ser. A 393 (1984), 277-295.

[24] 39 (1986), 297-307.

[25] _ Inverse problem for the euler-bernoulli beam, Proc. R. Soc. London A 407 (1986), 199-218.

[26] _ Inverse problems in vibration, Martinus Nijhoff Publishers, Dordrecht, 1989.

[27] _ Inverse vibration problems for finite elements models, Inverse Problem 3 (1997), $311-322$.

[28] _ Inverse finite element vibration problems, Preprint (1999). 
[29] G.M.L. Gladwell e J.A. Gbadeyan, On the inverse problem of the vibrating string or rod, Quart. J. Mech. Appl. Math. 38 (1985), 169-174.

[30] G.M.L. Gladwell, N.B. Willms, B. He e D. Wang, How can we recognize an aceptable mode shape for a vibrating beam?, Q. J. Mech. Appl. Math. 42 (1989), 303-316.

[31] G.H. Golub e D. Boley, Inverse eigenvalue problems for band matrices, Numerical Analysis (1977), no. 66, 23-31.

[32] G.H. Golub e C.F. Van Loan, Matrix computations, The Johns Hopkins University Press, Baltimore, 1989.

[33] W.B. Gragg e J.H. Welsch, The numerically stable reconstruction of jacobi matrices from spectral data, Numer. Math. 44 (1984), 317-335.

[34] L.J. Gray e D.G. Wilson, Construction of a jacobi matrix from spectral data, Lin. Alg. Appl. 14, 131-134.

[35] O. Hald, Inverse eigenvalue problems for jacobi matrices, Lin. Alg. Appl. 14 (1976), 63-85.

[36] G. Hearn e R.B. Testa, Modal analysis for damage detection in structures, J. Struct. Eng. 117 (1991), 3042-3063.

[37] H. Hochstadt, On construction of a jacobi matrix from spectral data, Lin. Alg. Appl. 8 (1974), 435-446.

[38] K.T. Joseph, Inverse eigenvalue problem in structural design, AIAA Ed. Ser. 30, 2890-2896.

[39] R. Knobel e J.R. McLaughlin, A reconstruction method for a two-dimensional inverse eigenvalue problem, Z. Angew. Math. Phys. 18 (1994), 794-826.

[40] C. Lanczos, An iteration method for the solution of the eigenvalue problem of linear differential and integral operators, J res NBS Section B 45 (1950).

[41] J.R. McLaughlin e H. Hald, A formula for finding a potencial from nodal lines, Bull. Amer. Math. Soc., New Series 32 (1995), 241-247.

[42] J.R. McLaughlin, P.L. Polyakov e P.E. Sacks, Reconstruction of a spherically symmetric speed of sound, SIAM J. Appl. MAth. 54 (1994), 1203-1223.

[43] A. Morassi, Crack-induced changes in eigenparameter of beam structures, J. Eng. Mech. 119 (1993), 1798-1803.

[44] P. Nylen e F. Uhlig, Inverse eigenvalue problems: existence of special spring-mass systems, Inverse Problems 13 (1997), 1071-1081. 
[45] B.N. Parllet. The symmetric eigenvalue problem, Englewood Cliffs, N J: Prentice Hall, 1980.

[46] W.H. Press, S.A. Teukolsky W.T. Vetterling e B.P. Flannery, Numerical recipes in fortran 77, vol. 1, Cambridge University Press, 1992.

[47] Y.M. Ram, Inverse eigenvalue problems for a modified vibrating system, SIAM J Appl 53 (1993), 1762-1775.

[48] Y.M. Ram e J. Caldwell, Phiysical parameters reconstruction of a free-free mass-spring system from its spectra, SIAM J. Appl. MAth. 52 (1992), 140-152.

[49] Y.M. Ram e S. Elhay, The construction of band symmetric models for vibratory systems from modal analysis data, J. Sound Vibration 181 (1995), 583-594.

[50] _ An inverse eigenvalue problem for the symmetric tridiagonal quadratic pencil with application to damped oscillatory systems, SIAM J. Appl. MAth. 56 (1996), 232-244.

[51] Y.M. Ram e G.M.L. Gladwell, Constructing a finite element model of a vibratory rod form eigendata, J. Sound Vibration 169 (1994), 229-237.

[52] M.H.H. Shen e J.E. Taylor, Indentification problem for vibrating cracked beams, J. Sound Vib. 150 (1991), 457-484.

[53] G.D. Shepard e J. Milani, Frequency based localization of structural discrepancies, Mech. Syst. Signal Processing 4, 173-184.

[54] J. Stoer e R. Bulirsh, Introduction to numerical analysis, second ed., Springer Verlag, 1980.

[55] M.A.M. Torkamani e A.K.Ahmadi, Stiffness identification of frames using simulated ground excitation, J. Eng. Mech. 114 (1988), 753-776.

[56] F.E. Udwadia, J.A. Garba e A. Ghodsi, Parameter identification problems in structural and geothecnical engineering, J. Eng. Mech. 110 (1984), 1409-1432.

[57] G.N. Vanderplaats, Numerical optimization techniques for engineering design with applications, McGraw-Hill, Inc., 1984.

[58] Q. Wu, Determination of the size of an object and its location in cavity by eigenfrequency shifts, Ph.D. thesis, Univ of Sydney, 1990.

[59] _ Reconstruction of integrated crack function of beams from eigenvalue shifts, $J$. Sound Vib. 173 (1994), 279-282.

[60] Q. Wu e F. Fricke, Estimation of blockage dimensions in a using measured eigenfrequency shifts, J. Sound Vib. 113 (1989). 
[61] _ Determination of bolckage locations and cross-sectional area in a duct by eigenfrequency shifts, J. Acoust Soc. Am. 87 (1990), 67-75.

[62] _ Determination of the size of an object and its location in a rectangular cavity by eigenfrequency shifts, J. Sound Vib. 144 (1991), 131-147.

[63] E.M.E. Zayed, An inverse eigenvalue problem for an arbitrary multiply connected bounded region in, Internat. J. Math. Math. Sci. 14 (1991), 571-579.

[64] _ An inverse eigenvalue problem for an arbitrary multiply connected bounded region in with independence boundary conditions, SIAM J. Appl. Math. 52 (1992), 725-729.

[65] _ An inverse eigenvalue problem for an arbitrary multiply connected bounded region: An extension to higher dimensions, Internat. J. Math. Math. Sci. 16 (1993), 485-492. 